\title{
Concise UC Zero-Knowledge Proofs for Oblivious Updatable Databases
}

\author{
Jan Camenisch*, Maria Dubovitskaya*, Alfredo Rial ${ }^{\dagger}$ \\ ${ }^{*}$ Dfinity, Zurich, Switzerland \\ Email: \{jan,maria\}@dfinity.org \\ ${ }^{\dagger}$ SnT, University of Luxembourg, Esch-sur-Alzette, Luxembourg \\ Email: alfredo.rial@uni.lu
}

\begin{abstract}
We propose an ideal functionality $\mathcal{F}_{\mathrm{CD}}$ and a construction $\Pi_{\mathrm{CD}}$ for oblivious and updatable committed databases. $\mathcal{F}_{\mathrm{CD}}$ allows a prover $\mathcal{P}$ to read, write, and update values in a database and to prove to a verifier $\mathcal{V}$ in zero-knowledge (ZK) that a value is read from or written into a certain position. The following properties must hold: (1) values stored in the database remain hidden from $\mathcal{V}$; (2) a value read from a certain position is equal to the value previously written into that position; (3) (obliviousness) both the value read or written and its position remain hidden from $\mathcal{V}$.

$\Pi_{C D}$ is based on vector commitments. After the initialization phase, the cost of read and write operations is independent of the database size, outperforming other techniques that achieve cost sublinear in the dataset size for prover and/or verifier. Therefore, our construction is especially appealing for large datasets.

In existing "commit-and-prove" two-party protocols, the task of maintaining a committed database between $\mathcal{P}$ and $\mathcal{V}$ and reading and writing values into it is not separated from the task of proving statements about the values read or written. $\mathcal{F}_{\mathrm{CD}}$ allows us to improve modularity in protocol design by separating those tasks. In comparison to simply using a commitment scheme to maintain a committed database, $\mathcal{F}_{\mathrm{CD}}$ allows $\mathcal{P}$ to hide efficiently the positions read or written from $\mathcal{V}$. Thanks to this property, we design protocols for e.g. privacy-preserving e-commerce and location-based services where $\mathcal{V}$ gathers aggregate statistics about the statements that $\mathcal{P}$ proves in $\mathrm{ZK}$.
\end{abstract}

Index Terms-Vector commitments, $\mathrm{ZK}$ proofs of knowledge, universal composability

\section{INTRODUCTION}

In protocols that use a commit-and-prove methodology (e.g. [1]), the prover $\mathcal{P}$ commits to her input and then proves in zero-knowledge (ZK) to a verifier $\mathcal{V}$ statements about the committed values. These steps are repeated and intertwined, i.e., commitments are updated, new ones formed, and additional proofs executed.

We regard commitments as a tool used to maintain a database of values between $\mathcal{P}$ and $\mathcal{V}$. When $\mathcal{P}$ commits to a value, the value is written into the database. When $\mathcal{P}$ proves a statement about a value committed previously, $\mathcal{P}$ reads a value from the database. Commitments guarantee the following properties: (1) values stored in the database are hidden from $\mathcal{V}$ (hiding property); (2) once a value is written into the database at a certain position (i.e, commitment), $\mathcal{P}$ cannot read a different value (binding property); (3) ZK proofs for reading or writing a value ensure that the value remains hidden from $\mathcal{V}$.
The use of commitments to maintain a database between $\mathcal{P}$ and $\mathcal{V}$ is not adequate in protocols where it is necessary to hide not only the value read or written into the database, but also the position where a value is stored. Our main example are protocols that allow $\mathcal{V}$ to gather statistics about what $\mathcal{P}$ proves in ZK, which are of interest for e.g. privacy-preserving e-commerce [2], billing [3] or location sharing services [4]. For instance, in [4], the positions represent locations, and the values are counters on the number of times $\mathcal{P}$ visited a location. When $\mathcal{P}$ visits a location, $\mathcal{P}$ updates the database to increment the counter for that location. To protect $\mathcal{P}$ 's privacy, $\mathcal{V}$ must learn neither the location nor the counter value.

Unfortunately, if commitments were used to maintain the database, the cost of a $\mathrm{ZK}$ proof that meets those properties grows linearly with the database size. We would like that this cost be independent of the database size.

On the other hand, in commit-and-prove protocols, the task of maintaining a database between $\mathcal{P}$ and $\mathcal{V}$ and reading and writing values into it is not separated from the task of proving statements about the values read or written. I.e., typically $\mathcal{P}$ computes a ZK proof to prove a statement about a committed value. Such a proof involves both reading a value from the database and proving a statement about it.

We propose to separate the task of maintaining a database between $\mathcal{P}$ and $\mathcal{V}$ from the task of proving statements about the values read or written or about the positions where the values are stored. This will improve modularity in protocol design and will lead to simpler and more structured security proofs that are easier to verify. Additionally, it will enable the study of the task of maintaining a database between $\mathcal{P}$ and $\mathcal{V}$ in isolation, which allows an easy comparison of different techniques to maintain a database.

\section{A. Our Contribution}

UC functionality. In Section III, we define an ideal functionality $\mathcal{F}_{\mathrm{CD}}$ for an oblivious and updatable committed database (CD) in the universal composability (UC) framework. The database consists of a table $\mathrm{Tbl}_{c d}$ with $N_{\max }$ entries of the form $[i, v]$, where $i$ is a position in $\left[1, N_{\max }\right]$ and $v$ is the value stored at that position. $\mathcal{F}_{\mathrm{CD}}$ ensures the following:

1) The values in the database remain hidden from $\mathcal{V}$.

2) $\mathcal{V}$ is guaranteed that a value read from $\mathrm{Tbl}_{c d}$ at position $i$ is equal to the value previously written into $i$. 
3) (Obliviousness) In read and write operations, both the value and the position read or written are hidden from $\mathcal{V}$. The database is updatable because $\mathcal{F}_{\mathrm{CD}}$ allows $\mathcal{P}$ to overwrite values into the database at any time.

$\mathcal{F}_{\mathrm{CD}}$ allows $\mathcal{P}$ to read and write values into the database. To prove statements about a value, or about the position where the value is read or written, $\mathcal{P}$ must use an ideal functionality $\mathcal{F}_{\mathrm{ZK}}^{R}$ for zero-knowledge parameterized by the appropriate statement $R$. This effectively separates the task of maintaining a database from the task of proving statements about the positions and values read or written. Consequently, we are able to design constructions for the committed database task in isolation and to compare them.

In a hybrid protocol that uses $\mathcal{F}_{\mathrm{CD}}$ and $\mathcal{F}_{\mathrm{ZK}}^{R}$ as building blocks, we need to guarantee that the value and the position read or written into $\mathcal{F}_{\mathrm{CD}}$ are equal to the value and position sent to $\mathcal{F}_{\mathrm{ZK}}^{R}$. For this purpose, we use a method proposed in [5], which consists in sending committed inputs to $\mathcal{F}_{\mathrm{CD}}$ and $\mathcal{F}_{\mathrm{ZK}}^{R}$ (see Section II).

Construction $\Pi_{\mathrm{CD}}$. In Section $\mathrm{V}$, we provide an efficient construction $\Pi_{\mathrm{CD}}$ for $\mathcal{F}_{\mathrm{CD}}$ that uses vector commitments (VC) [6], [7], which are suitable to implement a database $\mathrm{Tbl}_{c d}$ that consists of a one-dimensional array. A VC is a type of commitment that allows committing to a vector of values. The committer can open single positions of the committed vector to $\mathcal{V}$ with communication cost constant and independent of the vector size. VCs can also be updated. The computation cost of updating a commitment $v c$ or a witness $w$ to open a position grows linearly only with the number of updates and does not depend on the size of the committed vector.

$\Pi_{\mathrm{CD}}$ works as follows. At setup, $\mathcal{P}$ and $\mathcal{V}$ map the initial values of $\mathrm{Tbl}_{c d}$ to a vector of length $N_{\max }$ and compute a vector commitment $v c$ to that vector. When $\mathcal{P}$ wants to read the value $v$ at position $i, \mathcal{P}$ computes a VC witness $w$ for position $i$ and proves in $\mathrm{ZK}$ that $v$ is committed at position $i$. To write a value $v$ at a position $i$ into the database, $\mathcal{P}$ updates $v c$ to $v c^{\prime} . v c^{\prime}$ commits to the same vector as $v c$, except that $v$ is now at position $i$. Then $\mathcal{P}$ sends $v c^{\prime}$ to $\mathcal{V}$ and proves in $\mathrm{ZK}$ that $v c^{\prime}$ is an update of $v c$.

The communication cost of read and write operations is independent of the vector length $N_{\max }$. The cost of computing $v c$ and $w$ grows linearly with $N_{\max }$. However, we note that $v c$ and $w$ only need to be computed once. After that, they can be reused for multiple read operations and, when the database is updated, $v c$ and $w$ can be updated with cost that grows with the number of updates, but that is independent of $N_{\max }$. Therefore $\Pi_{C D}$ is suitable for large databases. In Section IX, we show that our construction improves in terms of efficiency over the existing $\mathrm{ZK}$ protocols for proving statements that involve large witnesses, such as $\mathrm{ZK}$ proofs for relations described as ORAM programs [8], [9]. In Section VI, we propose an efficient instantiation of $\Pi_{C D}$ that uses a VC scheme secure under the DHE assumption.

Modular design and applications of $\mathcal{F}_{\mathrm{CD}}$. In Section VII, we describe how to design a hybrid protocol that uses $\mathcal{F}_{\mathrm{CD}}$ and $\mathcal{F}_{\mathrm{ZK}}^{R}$ following the method in [5]. $\mathcal{F}_{\mathrm{CD}}$ is particularly useful for protocols where $\mathcal{P}$ needs to hide from $\mathcal{V}$ the positions read or written into the database. (Otherwise, a simple commitment scheme could be used to store the database.) In Section VIII, we describe some applications where this is the case. First, we describe how to use our committed database to compute OR proofs, i.e. $\mathrm{ZK}$ proofs of a disjunction of statements, with amortized cost independent of the size of the witness. Second, we show how $\mathcal{F}_{\mathrm{CD}}$ can be used to design protocols for privacy-preserving e-commerce and location-based services where service providers can gather aggregate statistics about users. In those protocols, $\mathcal{F}_{\mathrm{CD}}$ is used to store counters on the number of times a user buys a type of item or checks in at a certain location. When a user purchases an item or checks in at a location, the user computes a $\mathrm{ZK}$ proof that uses the item or the location as witness. Then the corresponding counter in $\mathcal{F}_{\mathrm{CD}}$ is incremented. At a later stage, the user can read counters from $\mathcal{F}_{\mathrm{CD}}$ to prove that they satisfy a statistic of interest to the service provider. We formalize this use of $\mathcal{F}_{\mathrm{CD}}$ as "zero-knowledge counting", i.e., counting the number of times a witness value is used by a prover in different $\mathrm{ZK}$ proofs. Beyond "zero-knowledge counting", we discuss how $\mathcal{F}_{\mathrm{CD}}$ is useful for gathering statistics that are more involved than counting, such as consumption statistics in privacy-preserving billing protocols [3].

\section{B. Previous Work}

The work in [5] increased the capabilities of modular protocol design in the UC framework by introducing a new functionality for non-interactive commitments $\mathcal{F}_{\mathrm{NIC}}$. $\mathcal{F}_{\mathrm{NIC}}$ is used to guarantee that two or more functionalities receive the same input. Having $\mathcal{F}_{\mathrm{NIC}}$ as a separate functionality simplifies the composition of building blocks and the security analysis of higher-level protocols.

Many protocols (e.g. [10], [11]) use commitment schemes to maintain a database between $\mathcal{P}$ and $\mathcal{V}$. However, commitments are not adequate to realize $\mathcal{F}_{\mathrm{CD}}$ because they do not allow $\mathcal{P}$ to hide efficiently the positions read from or written into the database. This is necessary in privacy-preserving protocols that allow $\mathcal{V}$ to gather statistics about what $\mathcal{P}$ proves in $\mathrm{ZK}$, which are of interest in e-commerce [2], billing [3] or location sharing services [4].

The literature on commit-and-prove protocols [1] is very large. In the following, we restrict ourselves to discussing primitives that can be applied to construct a committed database that allows $\mathcal{P}$ to hide efficiently the positions read or written and to prove statements about those positions.

Vector commitments (VCs) [6], [7], [12], a type of functional commitment [13], are the mechanism implicitly used in [4] to maintain a database in such a way that $\mathcal{P}$ can hide efficiently the positions read or written and prove statements about them. Unlike commitments, VCs allow us to open a vector component with communication cost independent of the vector length.

ZK proofs of shuffles [14] can be used as a way of hiding positions read or written by shuffling data in the database at each read or write operation. A construction using 
commitments along with proofs of shuffles could realize the same functionality offered by VCs, but less efficiently.

Several data structures that offer ZK properties exist. Examples include ZK sets [6], [15]-[17], updatable ZK databases [7], [18], ZK lists [19], [20] and trees [20]. These constructions typically require the size of the data structure to be hidden, which is a property we usually do not need for the applications of committed databases (CD) that we consider in this paper. (Some works relax this property [12], [21], [22].) On the other hand, for CD it is fundamental that the data structure can be modified dynamically, which is provided only in [7], [18], [20]. We provide a detailed comparison between those constructions and our committed database in Section IX.

However, security for those $\mathrm{ZK}$ data structures has not been defined in a composable security framework. To design modularly protocols that need to maintain a database between $\mathcal{P}$ and $\mathcal{V}$, we could only use the ideal protocol for ZK proofs parameterized with different relations. These relations would describe both the way data is kept, read from and written into the database, as well as the statements proven about those data. Consequently, the modularity of the design is limited because complex ZK proofs for those relations cannot be decomposed into simpler ones and thus require a monolithic security analysis. The concept of CD will allow the security analysis of similar ZK data structures in a composable framework, which will facilitate the modular design and analysis of protocols that use them as a building block.

\section{Outline of the Paper}

We describe how to design UC protocols modularly in Section II. In Section III, we define our ideal functionality $\mathcal{F}_{\mathrm{CD}}$. In Section IV, we define the building blocks of our construction $\Pi_{C D}$ and, in Section $V$, we describe $\Pi_{C D}$. We describe an efficient instantiation of our construction in Section VI. In Section VII, we show how to use our functionality as a building block in the modular design of cryptographic protocols. In Section VIII, we describe applications for $\mathcal{F}_{\mathrm{CD}}$. We describe related work in Section IX and conclude and hint some future work in Section X.

\section{Modular Design And Ideal Functionality $\mathcal{F}_{\text {NIC }}$}

We summarize the UC framework in Appendix A. An ideal functionality can be invoked by using one or more interfaces. In the notation in [5], the name of a message in an interface consists of three fields separated by dots, e.g., cd.setup.ini in $\mathcal{F}_{\mathrm{CD}}$ in Section III. The first field indicates the name of $\mathcal{F}_{\mathrm{CD}}$ and is the same for all interfaces. This field is useful for distinguishing between invocations of different functionalities in a hybrid protocol. The second field indicates the kind of action performed by $\mathcal{F}_{\mathrm{CD}}$ and is the same in all messages that $\mathcal{F}_{\mathrm{CD}}$ exchanges within the same interface. The third field distinguishes between the messages that belong to the same interface, and can take the following values. A message cd.setup.ini is the incoming message received by $\mathcal{F}_{\mathrm{CD}}$, i.e., the message through which the interface is invoked. cd.setup.end is the outgoing message sent by $\mathcal{F}_{\mathrm{CD}}$, i.e., the message that ends the execution of the interface. cd.setup.sim is used by $\mathcal{F}_{\mathrm{CD}}$ to send a message to the simulator $\mathcal{S}$, and cd.setup.rep is used to receive a message from $\mathcal{S}$.

In the UC framework, protocols can be described modularly by using a hybrid model where parties invoke the ideal functionalities of the building blocks of a protocol. One challenge when describing a UC protocol in the hybrid model is to ensure, when needed, that two or more ideal functionalities receive the same input. To address this issue, we use the method proposed in [5]. In [5], a functionality $\mathcal{F}_{\text {NIC }}$ for non-interactive commitments is proposed. $\mathcal{F}_{\text {NIC }}$ interacts with parties $\mathcal{P}_{i}$ and consists of four interfaces com.setup, com.validate, com.commit and com.verify:

1) Any party $\mathcal{P}_{i}$ uses the com.setup interface to set up the functionality.

2) Any party $\mathcal{P}_{i}$ uses the com.commit interface to send a message $m$ and obtain a commitment com and an opening open. A commitment com consists of ( $\mathrm{com}^{\prime}$, parcom, COM.Verify), where $\mathrm{com}^{\prime}$ is the commitment, parcom are the public parameters, and COM.Verify is the verification algorithm.

3) Any party $\mathcal{P}_{i}$ uses the com.validate interface to send a commitment com in order to check that com contains the correct public parameters and verification algorithm.

4) Any party $\mathcal{P}_{i}$ uses the com.verify interface to send (com, $m$,open) in order to verify that com is a commitment to the message $m$ with the opening open.

$\mathcal{F}_{\text {NIC }}$ can be realized by a perfectly hiding commitment scheme, such as Pedersen commitments [5]. In [5], a method is described to use $\mathcal{F}_{\text {NIC }}$ in order to ensure that a party sends the same input $m$ to several ideal functionalities. For this purpose, the party first uses com.commit to get a commitment com to $m$ with opening open. Then the party sends (com, m,open) as input to each of the functionalities, and each functionality runs COM.Verify to verify the commitment. Finally, other parties in the protocol receive the commitment com from each of the functionalities and use the com.validate interface to validate $\mathrm{com}$. Then, if com received from all the functionalities is the same, the binding property provided by $\mathcal{F}_{\text {NIC }}$ ensures that all the functionalities received the same input $m$. When using $\mathcal{F}_{\mathrm{NIC}}$, it is needed to work in the $\mathcal{F}_{\mathrm{NIC}} \| \mathcal{S}_{\mathrm{NIC}}$-hybrid model, where $\mathcal{S}_{\text {NIC }}$ is any simulator for a construction that realizes $\mathcal{F}_{\text {NIC }}$. The reason is that we need to ensure that the output of COM.Verify is indistinguishable from the output of the com.verify interface of $\mathcal{F}_{\mathrm{NIC}}$. Our functionality $\mathcal{F}_{\mathrm{CD}}$ receives committed inputs as described in [5]. We depict $\mathcal{F}_{\mathrm{NIC}}$ in Appendix E.

\section{IDEAL FunCtionality $\mathcal{F}_{\mathrm{CD}}$}

Intuition. Our functionality $\mathcal{F}_{\mathrm{CD}}$ interacts with a prover $\mathcal{P}$ and a verifier $\mathcal{V}$ and has three interfaces: "setup", "read" and "write". $\mathcal{F}_{\mathrm{CD}}$ maintains a committed database where every entry is stored as a tuple [position, value]. The "setup" interface initializes the database to values known to both $\mathcal{P}$ and $\mathcal{V}$. The "write" interface allows $\mathcal{P}$ to update an entry of the committed database. $\mathcal{V}$ learns neither the position nor the value of the 
entry being written. The "read" interface allows $\mathcal{P}$ to prove to $\mathcal{V}$ knowledge of an entry in the database without revealing neither its position nor the value to $\mathcal{V}$. To ensure that both the prover and the verifier use the same version of the database, $\mathcal{F}_{\mathrm{CD}}$ maintains counters for the number of writing operations sent by $\mathcal{P}$ and the number of writing operations received by $\mathcal{V}$. The counters are checked for consistency for both read and write queries.

Both "read" and "write" interfaces require commitments to the position and to the value to be provided as input. This allows $\mathcal{F}_{\mathrm{CD}}$ to be used in conjunction with other functionalities (e.g. $\mathcal{F}_{\mathrm{ZK}}^{R}$ for $\mathrm{ZK}$ proofs of knowledge) in a modular way to build high-level protocols. That is, once the prover proves to the verifier that the commitment values commit to a database entry by using $\mathcal{F}_{\mathrm{CD}}$, those commitments can be used as input to other functionalities of the high-level (hybrid) protocol. For example, the commitment to the position and to the value can be input to $\mathcal{F}_{\mathrm{ZK}}^{R}$ to prove in $\mathrm{ZK}$ statements about the position and the value read from or written into the database. This allows us to prove different statements about the values from the database without revealing neither the value itself nor its position to the verifier. The binding property guaranteed by $\mathcal{F}_{\text {NIC }}$ ensures that the committed values given as input to $\mathcal{F}_{\mathrm{CD}}$ and to $\mathcal{F}_{\mathrm{ZK}}^{R}$ are equal.

Notation. $\mathcal{F}_{\mathrm{CD}}$ is parameterized by a universe of state values $U_{v}$ and by a state size $N_{\max } . \mathcal{F}_{\mathrm{CD}}$ maintains a table $\mathrm{Tbl}_{c d}$ that stores the database. $\mathrm{Tbl}_{c d}$ contains $N_{\max }$ entries of the form $[i, v]$, where $i \in\left[1, N_{\max }\right]$ is the position in the table and $v \in U_{v}$ is the value stored at that position. ( $N_{\max }$ needs to be fixed so that $\mathcal{F}_{\mathrm{CD}}$ can be realized by a construction based on VCs, whose set up algorithm needs knowledge of $\left.N_{\text {max }}.\right) \mathcal{F}_{\mathrm{CD}}$ maintains a counter $c p$ for the number of writing operations sent by $\mathcal{P}$ and a counter $c v$ for the number of writing operations received by $\mathcal{V}$. The interaction between the functionality $\mathcal{F}_{\mathrm{CD}}, \mathcal{P}$ and $\mathcal{V}$ takes place through the following interfaces:

- $\mathcal{V}$ uses the cd.setup interface to initialize $\mathrm{Tbl}_{c d} . \mathcal{F}_{\mathrm{CD}}$ stores $\mathrm{Tbl}_{c d}$ and sends $\mathrm{Tbl}_{c d}$ to $\mathcal{P}$ and to the simulator $\mathcal{S}$.

- $\mathcal{P}$ uses cd.read to send a position $i$ and a value $v_{r}$ to $\mathcal{F}_{\mathrm{CD}}$, along with commitments and openings $\left(\right.$ com $_{i}$, open $\left._{i}\right)$ and $\left(\right.$ com $_{r}$, open $\left._{r}\right)$ to the position and value respectively. $\mathcal{F}_{\mathrm{CD}}$ verifies the commitments and checks that there is an entry $\left[i, v_{r}\right]$ in the table $\mathrm{Tbl}_{c d}$. In that case, $\mathcal{F}_{\mathrm{CD}}$ sends com $_{i}$ and $\operatorname{com}_{r}$ to $\mathcal{V}$. $\mathcal{S}$ also learns $\operatorname{com}_{i}$ and $\operatorname{com}_{r}$.

- $\mathcal{P}$ uses cd.write to send a position $i$ and a value $v_{w}$ to $\mathcal{F}_{\mathrm{CD}}$, along with commitments and openings $\left(\right.$ com $_{i}$, open $\left._{i}\right)$ and $\left(\right.$ com $_{w}$, open $\left._{w}\right)$ to the position and value respectively. $\mathcal{F}_{\mathrm{CD}}$ verifies the commitments and then updates $\mathrm{Tbl}_{c d}$ to store $v_{w}$ at position $i . \mathcal{F}_{\mathrm{CD}}$ sends $\operatorname{com}_{i}$ and $\operatorname{com}_{w}$ to $\mathcal{V}$. $\mathcal{S}$ also learns $\operatorname{com}_{i}$ and $\operatorname{com}_{w}$.

The commitment parameters parcom and the commitment verification algorithm COM.Verify are included in the commitment values (as in functionality $\mathcal{F}_{\mathrm{NIC}}$ ).

We describe $\mathcal{F}_{\mathrm{CD}}$ in Figure 1. We consider static corruptions, i.e. parties can only be corrupted by the adversary at the beginning of the protocol execution. In this description, we list all the abortion conditions and describe how $\mathcal{F}_{\mathrm{CD}}$ saves its state before querying the simulator $\mathcal{S}$ and recovers it after receiving a response from $\mathcal{S}$. These steps are often omitted in the description of ideal functionalities. In the "read" and "write" interfaces, $\mathcal{F}_{\mathrm{CD}}$ creates a query identifier qid to link the replies from $\mathcal{S}$ to the corresponding query to $\mathcal{S}$. In the "setup" interface, this is not needed because that interface can only be invoked once.

When invoked by $\mathcal{V}$ or $\mathcal{P}, \mathcal{F}_{\mathrm{CD}}$ first checks the correctness of the input and aborts if it does not belong to the correct domain. $\mathcal{F}_{\mathrm{CD}}$ also aborts if an interface is invoked at an incorrect moment in the protocol. For example, $\mathcal{P}$ cannot invoke $c d$.read before $\mathcal{V}$ invokes cd.setup.

$\mathcal{F}_{\mathrm{CD}}$ also aborts if the commitment verification algorithms received as input are not ppt. This check is performed in the manner described in Section 7.2 paragraph "running arbitrary code" in [23].

Discussion of $\mathcal{F}_{\mathrm{CD}}$. The restriction that the identities $\mathcal{P}$ and $\mathcal{V}$ must be included in the session identifier sid $=\left(\mathcal{P}, \mathcal{V}\right.$, sid $\left.^{\prime}\right)$ guarantees that every verifier can create an instance of $\mathcal{F}_{\mathrm{CD}}$ with every prover. $\mathcal{F}_{\mathrm{CD}}$ implicitly checks that sid in a message equals the one received in the first invocation. We note that it is easy to modify $\mathcal{F}_{\mathrm{CD}}$ and our construction for $\mathcal{F}_{\mathrm{CD}}$ so that the setup phase is started by $\mathcal{P}$.

In the "read" interface, $\mathcal{F}_{\mathrm{CD}}$ aborts if $\left[i, v_{r}\right]$ received as input are not stored in $\mathrm{Tbl}_{c d}$. This guarantees to $\mathcal{V}$ that the position and the value committed to in $\mathrm{com}_{i}$ and in $\mathrm{com}_{r}$ respectively correspond to an entry in $\mathrm{Tbl}_{c d}$. After being triggered by $\mathcal{S}$, $\mathcal{F}_{\mathrm{CD}}$ aborts if the query identifier is not stored, or if the number of writing operations received by $\mathcal{V}$ does not equal the number of writing operations sent by $\mathcal{P}$ when the read operation was started. This guarantees that the table used by $\mathcal{P}$ when computing the read operation equals the table used by $\mathcal{V}$ to verify the read operation. A similar check is performed by $\mathcal{F}_{\mathrm{CD}}$ in the "write" interface.

$\mathcal{F}_{\mathrm{CD}}$ sends commitments to the position and to the value to $\mathcal{V}$. To hide the position and the value from $\mathcal{V}$, the hiding property of the commitment is required to hold. This is achieved by using $\mathcal{F}_{\mathrm{NIC}}$ to compute commitments.

\section{BUILDING BLOCKS}

Ideal Functionality $\mathcal{F}_{\mathrm{CRS}}^{\mathrm{CRS} \text {.Setup }}$. Our protocol uses the functionality $\mathcal{F}_{\mathrm{CRS}}^{\mathrm{CRS} \text { Setup }}$ for common reference string generation

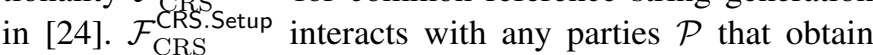
the common reference string, and consists of one interface crs.get. A party $\mathcal{P}$ uses the crs.get interface to request and receive the common reference string $c r s$ from $\mathcal{F}_{\mathrm{CRS}}^{\mathrm{CRS} \text {. Setup }}$. In the first invocation, $\mathcal{F}_{\text {CRS }}^{\text {CRS. Setup }}$ generates $c r s$ by running algorithm CRS.Setup. The simulator $\mathcal{S}$ also receives $c r s$. We depict

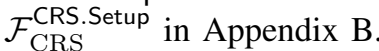

Ideal Functionality $\mathcal{F}_{\mathrm{AUT}}$. Our protocol uses the functionality $\mathcal{F}_{\text {AUT }}$ for an authenticated channel in [24]. $\mathcal{F}_{\text {AUT }}$ interacts with a sender $\mathcal{T}$ and a receiver $\mathcal{R}$, and consists of one interface aut.send. $\mathcal{T}$ uses the aut.send interface to send a message $m$ to $\mathcal{F}_{\text {AUT }} . \mathcal{F}_{\text {AUT }}$ leaks $m$ to the simulator $\mathcal{S}$ and, after receiving a response from $\mathcal{S}, \mathcal{F}_{\text {AUT }}$ sends $m$ to $\mathcal{R}$. $\mathcal{S}$ cannot modify $m$. 
Functionality $\mathcal{F}_{\mathrm{CD}}$ is parameterized by a universe of values $U_{v}$ and by a maximum table size $N_{\max } . \mathcal{F}_{\mathrm{CD}}$ interacts with a prover $\mathcal{P}$ and a verifier $\mathcal{V}$.

1) On input (cd.setup.ini, sid, $\mathrm{Tbl}_{c d}$ ) from $\mathcal{V}$ :

- Abort if $\operatorname{sid} \notin\left(\mathcal{P}, \mathcal{V}, s i d^{\prime}\right)$ or if $\left(\right.$ sid, $\left.\mathrm{Tbl}_{c d}\right)$ is already stored.

- Abort if $\mathrm{Tbl}_{c d}$ does not consist of entries of the form $[i, v]$, or if the number of entries in $\mathrm{Tbl}_{c d}$ is not $N_{\max }$.

- Abort if for $i=1$ to $N_{\max }, v \notin U_{v}$ for any entry $[i, v]$ in $\mathrm{Tbl}_{c d}$.

- Initialize a counter $c v \leftarrow 0$ for the verifier and store $($ sid, $c v)$ and $\left(\right.$ sid, $\left.\mathrm{Tbl}_{c d}\right)$.

- Send (cd.setup.sim, sid, $\mathrm{Tbl}_{c d}$ ) to $\mathcal{S}$.

S. On input (cd.setup.rep, sid) from $\mathcal{S}$ :

- Abort if $\left(s i d, \mathrm{Tbl}_{c d}\right)$ is not stored, or if $\left(s i d, \mathrm{Tbl}_{c d}, c p\right)$ is already stored.

- Initialize a counter $c p \leftarrow 0$ for the prover and store $\left(s i d, \mathrm{Tbl}_{c d}, c p\right)$.

- Send (cd.setup.end, sid, $\mathrm{Tbl}_{c d}$ ) to $\mathcal{P}$.

2) On input (cd.read.ini, sid, $\operatorname{com}_{i}, i$, open $_{i}, \mathrm{com}_{r}, v_{r}$, open $_{r}$ ) from $\mathcal{P}$ :

- Abort if $\left(s i d, \mathrm{Tbl}_{c d}, c p\right)$ is not stored.

- Abort if $i \notin\left[1, N_{\max }\right]$, or if $v_{r} \notin U_{v}$, or if $\left[i, v_{r}\right]$ is not stored in $\mathrm{Tbl}_{c d}$.

- Parse the commitment $\operatorname{com}_{i}$ as $\left(\operatorname{com}_{i}^{\prime}\right.$, parcom $_{i}$, COM.Verify $\left._{i}\right)$.

- Parse the commitment $\operatorname{com}_{r}$ as $\left(\text { com }_{r}^{\prime}, \text { parcom }_{r}, \text { COM.Verify }\right)_{r}$.

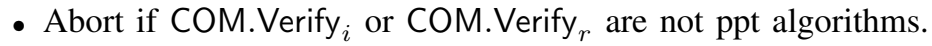

- Abort if $1 \neq$ COM.Verify $\left(\right.$ parcom $_{i}$, com $_{i}^{\prime}, i$, open $\left._{i}\right)$.

- Abort if $1 \neq$ COM.Verify $_{r}\left(\right.$ parcom $_{r}$, com $_{r}^{\prime}, v_{r}$, open $\left._{r}\right)$.

- Create a fresh qid and store (qid, $\left.c^{\circ} m_{i}, c m_{r}, c p\right)$.

- Send (cd.read.sim, sid, qid, $\operatorname{com}_{i}, \operatorname{com}_{r}$ ) to $\mathcal{S}$.

S. On input (cd.read.rep, sid, qid) from $\mathcal{S}$ :

- Abort if $\left(q i d, c o m_{i}, c o m_{r}, c p^{\prime}\right)$ is not stored.

- Abort if $c p^{\prime} \neq c v$, where $c v$ is stored in (sid, $\left.c v\right)$.

- Delete the record $\left(q i d, c^{\circ} m_{i}, c o m_{r}, c p^{\prime}\right)$.

- Send (cd.read.end, sid, $\operatorname{com}_{i}, \operatorname{com}_{r}$ ) to $\mathcal{V}$.

3) On input (cd.write.ini, sid, com $_{i}, i$, open $_{i}$, com $_{w}, v_{w}$, open $_{w}$ ) from $\mathcal{P}$ :

- Abort if $\left(s i d, \mathrm{Tbl}_{c d}, c p\right)$ is not stored.

- Abort if $i \notin\left[1, N_{\max }\right]$, or if $v_{w} \notin U_{v}$.

- Parse the commitment $\operatorname{com}_{i}$ as $\left(\operatorname{com}_{i}^{\prime}\right.$, parcom $_{i}$, COM.Verify $\left._{i}\right)$.

- Parse the commitment $\operatorname{com}_{w}$ as $\left(\operatorname{com}_{w}^{\prime}, \operatorname{parcom}_{w}, \mathrm{COM}_{\text {.Verify }}{ }_{w}\right)$.

- Abort if COM.Verify or COM.Verify $_{w}$ are not ppt algorithms.

- Abort if $1 \neq$ COM.Verify $\left(\right.$ parcom $_{i}$, com $_{i}^{\prime}, i$, open $\left._{i}\right)$.

- Abort if $1 \neq$ COM.Verify $\left(\right.$ arcom $_{w}, \operatorname{com}_{w}^{\prime}, v_{w}$, open $\left._{w}\right)$.

- Increment the counter $c p$ in $\left(s i d, \mathrm{Tbl}_{c d}, c p\right)$ and store $\left[i, v_{w}\right]$ in $\mathrm{Tbl}_{c d}$.

- Create a fresh qid and store (qid, $\left.c^{\circ} m_{i}, c m_{w}, c p\right)$.

- Send (cd.write.sim, sid, qid, $\operatorname{com}_{i}, \operatorname{com}_{w}$ ) to $\mathcal{S}$.

S. On input (cd.write.rep, sid, qid) from $\mathcal{S}$ :

- Abort if $\left(q i d, c^{\circ} m_{i}, c o m_{w}, c p^{\prime}\right)$ is not stored.

- Abort if $c p^{\prime} \neq c v+1$, where $c v$ is stored in ( $\left.s i d, c v\right)$.

- Increment the counter $c v$ in $(s i d, c v)$.

- Delete the record ( $\left.q i d, c^{\circ} m_{i}, c o m_{w}, c p^{\prime}\right)$.

- Send (cd.write.end, sid, $\operatorname{com}_{i}, \operatorname{com}_{w}$ ) to $\mathcal{V}$.

Fig. 1. Functionality $\mathcal{F}_{\mathrm{CD}}$ 
The session identifier sid contains the identities of $\mathcal{T}$ and $\mathcal{R}$. We depict $\mathcal{F}_{\text {AUT }}$ in Appendix $\mathrm{C}$.

Ideal Functionality $\mathcal{F}_{\mathrm{ZK}}^{R}$. Let $R$ be a polynomial time computable binary relation. For tuples (wit, ins) $\in R$ we call wit the witness and ins the instance. Our protocol uses the ideal functionality $\mathcal{F}_{\mathrm{ZK}}^{R}$ for zero-knowledge in [24]. $\mathcal{F}_{\mathrm{ZK}}^{R}$ is parameterized by a description of a relation $R$, runs with a prover $\mathcal{P}$ and a verifier $\mathcal{V}$, and consists of one interface zk.prove. $\mathcal{P}$ uses zk.prove to send a witness wit and an instance ins to $\mathcal{F}_{\mathrm{ZK}}^{R} \cdot \mathcal{F}_{\mathrm{ZK}}^{R}$ checks whether $($ wit, ins $) \in R$, and, in that case, sends the instance ins to $\mathcal{V}$. The simulator $\mathcal{S}$ learns ins but not wit. We depict $\mathcal{F}_{\mathrm{ZK}}^{R}$ in Appendix D.

Vector Commitments. Vector commitments (VC) [6], [7] allow us to commit to a vector of messages and to open the commitment to one of the messages in such a way that the size of the witness is independent of the length of the vector. A VC scheme consists of the following algorithms.

VC.Setup $\left(1^{k}, \ell\right)$. On input the security parameter $1^{k}$ and an upper bound $\ell$ on the size of the vector, generate the parameters of the commitment scheme par, which include a description of the message space $\mathcal{M}$ and a description of the randomness space $\mathcal{R}$.

VC.Commit $($ par $, \mathbf{x}, r)$. On input a vector $\mathbf{x} \in \mathcal{M}^{n}(n \leq \ell)$ and $r \in \mathcal{R}$, output a commitment $v c$ to $\mathbf{x}$.

VC.Wit $(p a r, i, \mathbf{x}, r)$. Compute a witness $w$ for $\mathbf{x}[i]$.

VC.Verify $(p a r, v c, x, i, w)$. Output 1 if $w$ is a valid witness for $x$ being at position $i$ and 0 otherwise.

VC.ComUpd $\left(p a r, v c, j, x, r, x^{\prime}, r^{\prime}\right)$. On input a commitment $v c$ with value $x$ at position $j$ and randomness $r$, output a commitment $v c^{\prime}$ with value $x^{\prime}$ at position $j$ and randomness $r^{\prime}$. The other positions remain unchanged.

VC.VerComUpd $\left(p a r, v c, v c^{\prime}, w, j, x, r, x^{\prime}, r^{\prime}\right)$. On input commitments $v c$ and $v c^{\prime}$, a witness $w$, a position $j$, the values $x$ and $x^{\prime}$ and the randomness $r$ and $r^{\prime}$, output 1 if $w$ is a valid witness for $x$ being at position $j$ in the commitment $v c$ and if $v c^{\prime}$ is an update of $v c$ that replaces $x$ by $x^{\prime}$ at position $j$ and $r$ by $r^{\prime}$.

VC.WitUpd $\left(\right.$ par $\left., w, i, j, x, r, x^{\prime}, r^{\prime}\right)$. On input a witness $w$ for a position $i$ valid for a commitment $v c$ with value $x$ at position $j$ and randomness $r$, output a witness $w^{\prime}$ for position $i$ valid for a commitment $v c^{\prime}$ with value $x^{\prime}$ at position $j$ and randomness $r^{\prime}$.

A VC scheme must be correct, hiding, and binding, as defined in Appendix G.

\section{Construction $\Pi_{\mathrm{CD}}$ FOR a Committed Database}

Our construction $\Pi_{\mathrm{CD}}$ in Figure 2 and Figure 3 uses a VC scheme. A vector commitment $v c$ is used to store the table $\mathrm{Tbl}_{c d}$. A position in the vector commitment acts as a position in $\mathrm{Tbl}_{c d}$, and the value committed to in that position acts as the value stored in $\mathrm{Tbl}_{c d}$ in that position.

In the setup interface, $\mathcal{P}$ and $\mathcal{V}$ obtain the VC parameters par from the functionality $\mathcal{F}_{\mathrm{CRS}}^{\mathrm{CRS} \text { Setup }}$ for common reference string, which is parameterized by the setup algorithm VC.Setup = CRS.Setup of the VC scheme. $\mathcal{V}$ receives as input a table $\mathrm{Tbl}_{c d}$ an computes a commitment $v c$ to $\mathrm{Tbl}_{c d}$ with 0 randomness. $\mathcal{V}$ sends $\mathrm{Tbl}_{c d}$ to $\mathcal{P}$ by using the ideal functionality $\mathcal{F}_{\mathrm{AUT}}$ for an authenticated channel, and $\mathcal{P}$ also computes a commitment $v c$ to $\mathrm{Tbl}_{c d}$ with 0 randomness.

In the read interface, $\mathcal{P}$ receives as input a position $i$ and a value $v_{r}$, along with commitments and openings $\left(\right.$ com $_{i}$, open $\left._{i}\right)$ and $\left(\right.$ com $_{r}$, open $\left._{r}\right) . \mathcal{P}$ uses the ideal functionality $\mathcal{F}_{\mathrm{ZK}}^{R_{r}}$ for ZK proofs of knowledge to prove to $\mathcal{V}$ that $\operatorname{com}_{i}$ and $\mathrm{com}_{r}$ commit to $i$ and $v_{r}$ such that $v_{r}$ is the message committed at position $i$ in the commitment $v c$. This proves that $\left[i, v_{r}\right] \in$ $\mathrm{Tbl}_{c d}$.

In the write interface, $\mathcal{P}$ receives as input a position $i$ and a value $v_{w}$, along with commitments and openings $\left(\mathrm{com}_{i}\right.$, open $\left._{i}\right)$ and $\left(\right.$ com $_{w}$, open $\left._{w}\right) . \mathcal{P}$ updates the commitment $v c$ to a commitment $v c^{\prime}$ that commits to $v_{w}$ at position $i$, while other positions remain unchanged. $\mathcal{P}$ uses the functionality $\mathcal{F}_{\mathrm{ZK}}^{R_{w}}$ to prove to $\mathcal{V}$ that $\operatorname{com}_{i}$ and $\operatorname{com}_{w}$ commit to $i$ and $v_{w}$, and that $v c^{\prime}$ is an update of $v c$ where $v_{w}$ is committed in the position $i$. We note that $v c^{\prime}$ contains randomness chosen by $\mathcal{P}$ and thus, after the first execution of the write interface, the committed table will be hidden from $\mathcal{V}$.

We describe $\Pi_{C D}$ in Figure 2 and Figure 3. For brevity, we omit some abortion conditions or the messages sent to the functionalities used as building blocks. The full description is in Appendix $\mathrm{H}$.

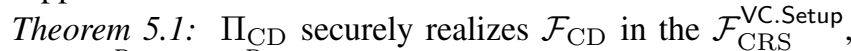
$\mathcal{F}_{\mathrm{AUT}}, \mathcal{F}_{\mathrm{ZK}}^{R_{r}}$ and $\mathcal{F}_{\mathrm{ZK}}^{R_{w}}$-hybrid model if the VC scheme is hiding and binding.

When $\mathcal{P}$ is corrupt, the binding property of the VC scheme guarantees that the adversary is not able to open the vector commitment to a position and a value if that value was not previously committed at that position. When $\mathcal{V}$ is corrupt, the hiding property of the VC scheme guarantees that the committed vector remains hidden from $\mathcal{V}$. We analyze in detail the security of $\Pi_{C D}$ in Appendix $\mathrm{I}$.

\section{EFFicient Instantiation of COnstruction $\Pi_{\mathrm{CD}}$}

Our instantiation of $\Pi_{C D}$ is based on a VC scheme secure under the DHE assumption and on $\mathrm{ZK}$ proofs of knowledge for the relations $R_{r}$ and $R_{w}$ for that $\mathrm{VC}$ scheme. To compute those proofs, the VC scheme is extended with a structurepreserving signature scheme. We use Pedersen commitments as the commitment scheme used to realize $\mathcal{F}_{\text {NIC }}$.

In Section VI-A, we describe the building blocks of our instantiation. In Section VI-B, we describe the ZK proofs for relations $R_{r}$ and $R_{w}$. We analyze the efficiency of our instantiation in Section VI-C.

\section{A. Building blocks of Our Instantiation}

Bilinear maps. Let $\mathbb{G}, \tilde{\mathbb{G}}$ and $\mathbb{G}_{t}$ be groups of prime order $p$. A map $e: \mathbb{G} \times \tilde{\mathbb{G}} \rightarrow \mathbb{G}_{t}$ must satisfy bilinearity, i.e., $e\left(g^{x}, \tilde{g}^{y}\right)=$ $e(g, \tilde{g})^{x y}$; non-degeneracy, i.e., for all generators $g \in \mathbb{G}$ and $\tilde{g} \in \widetilde{\mathbb{G}}, e(g, \tilde{g})$ generates $\mathbb{G}_{t}$; and efficiency, i.e., there exists an efficient algorithm $\mathcal{G}\left(1^{k}\right)$ that outputs the pairing group setup $g r p \leftarrow\left(p, \mathbb{G}, \widetilde{\mathbb{G}}, \mathbb{G}_{t}, e, g, \tilde{g}\right)$ and an efficient algorithm to compute $e(a, b)$ for any $a \in \mathbb{G}, b \in \tilde{\mathbb{G}}$. 
$\Pi_{\mathrm{CD}}$ uses a VC scheme and the functionalities $\mathcal{F}_{\mathrm{CRS}}^{\mathrm{VC} \text {.Setup }}, \mathcal{F}_{\mathrm{AUT}}, \mathcal{F}_{\mathrm{ZK}}^{R_{r}}$ and $\mathcal{F}_{\mathrm{ZK}}^{R_{w}}$. The table size $N_{\text {max }}$ is the maximum length of a committed vector, and the universe of values $U_{v}$ is given by the message space of the $\mathrm{VC}$ scheme.

1) On input (cd.setup.ini, sid, $\mathrm{Tbl}_{c d}$ ), $\mathcal{V}$ and $\mathcal{P}$ do the following:

- $\mathcal{V}$ uses the crs.get interface of $\mathcal{F}_{\mathrm{CRS}}^{\mathrm{VC} \text {.Setup }}$ to obtain the VC parameters par.

- $\mathcal{V}$ initializes a counter $c v \leftarrow 0$ that counts the write operations received.

- $\mathcal{V}$ stores $\mathrm{Tbl}_{c d}$ in a vector $\mathbf{x}$ : for $i=1$ to $N_{\max }, \mathbf{x}[i]=v$, where $[i, v] \in \operatorname{Tbl}_{c d}$.

- $\mathcal{V}$ commits to $\mathbf{x}$ : set $r \leftarrow 0$ and run $v c \leftarrow$ VC.Commit $(p a r, \mathbf{x}, r)$.

- $\mathcal{V}$ stores $($ sid, $c v$, par, $v c)$.

- $\mathcal{V}$ uses the aut.send interface of $\mathcal{F}_{\mathrm{AUT}}$ to send $\mathrm{Tbl}_{c d}$ to $\mathcal{P}$.

- $\mathcal{P}$ follows the same steps as $\mathcal{V}$ to get par, set $\mathbf{x}$ and compute $v c$.

- $\mathcal{P}$ initializes a counter $c p \leftarrow 0$ that counts write operations started.

- $\mathcal{P}$ stores (sid, $c p$, par, $v c, \mathbf{x}, r)$.

- $\mathcal{P}$ outputs (cd.setup.end, sid, $\mathrm{Tbl}_{c d}$ ).

2) On input (cd.read.ini, sid com $_{i}, i$, open $_{i}, \operatorname{com}_{r}, v_{r}$, open $_{r}$ ), $\mathcal{P}$ and $\mathcal{V}$ do:

- $\mathcal{P}$ parses $\operatorname{com}_{i}$ as $\left(\operatorname{com}_{i}^{\prime}\right.$, parcom $_{i}$, COM.Verify $\left._{i}\right)$.

- $\mathcal{P}$ parses com $_{r}$ as $\left(\right.$ comr $_{r}^{\prime}$, parcom $_{r}$, COM.Verify $\left.r\right)$.

- $\mathcal{P}$ takes the stored tuple (sid, $c p, p a r, v c, \mathbf{x}, r)$.

- If $(\operatorname{sid}, i, w)$ is not stored, $\mathcal{P}$ computes a $\mathrm{VC}$ witness $w$ for position $i$ : $\operatorname{run} w \leftarrow \operatorname{VC}$.Wit $($ par, $i, \mathbf{x}, r)$ and store $($ sid, $i, w)$.

- $\mathcal{P}$ sets wit $_{r} \leftarrow\left(w, i\right.$, open $_{i}, v_{r}$, open $\left._{r}\right)$.

- $\mathcal{P}$ sets ins $_{r} \leftarrow\left(\right.$ par, vc, parcom $_{i}$, com $_{i}^{\prime}$, parcom $_{r}$, com $\left._{r}^{\prime}, c p\right)$.

- $\mathcal{P}$ uses the zk.prove interface to send wit $t_{r}$ and $i n s_{r}$ to $\mathcal{F}_{\mathrm{ZK}}^{R_{r}}$, where $R_{r}$ is

$$
\begin{aligned}
R_{r}= & \left\{\left(\text { wit }_{r}, \text { ins }_{r}\right):\right. \\
& 1=\text { COM.Verify }_{i}\left(\text { parcom }_{i}, \text { com }_{i}^{\prime}, i, \text { open }_{i}\right) \wedge \\
& 1=\text { COM.Verify }_{r}\left(\text { parcom }_{r}, \text { com }_{r}^{\prime}, v_{r}, \text { open }_{r}\right) \wedge \\
& \left.1=\operatorname{VC} . \text { Verify }\left(\text { par }, v c, v_{r}, i, w\right)\right\}
\end{aligned}
$$

In equation $1, \mathcal{P}$ proves that $\operatorname{com}_{i}^{\prime}$ is a commitment to $i$ with opening $\operatorname{open}_{i}$. Similarly, in equation 2 , $\mathcal{P}$ proves that $\operatorname{com}_{r}^{\prime}$ is a commitment to $v_{r}$ with opening open $_{r}$. In equation $3, \mathcal{P}$ proves that $v_{r}$ is stored in the position $i$ of the vector commitment $v c$.

- $\mathcal{V}$ receives ins $_{r}=\left(\right.$ par $^{\prime}, v c^{\prime}$, parcom $_{i}$, com $_{i}^{\prime}$, parcom $_{r}$, com $\left._{r}^{\prime}, c p\right)$.

- $\mathcal{V}$ takes the stored tuple $($ sid, $c v, p a r, v c)$.

- $\mathcal{V}$ aborts if $c p \neq c v$, or if $p a r^{\prime} \neq p a r$, or if $v c^{\prime} \neq v c$.

- $\mathcal{V}$ sets $\operatorname{com}_{i} \leftarrow\left(\right.$ com $_{i}^{\prime}$, parcom $_{i}$, COM.Verify $\left._{i}\right)$.

- $\mathcal{V}$ sets com $_{r} \leftarrow\left(\right.$ com $_{r}^{\prime}$, parcom $_{r}$, COM.Verify $\left.r\right)$.

- $\mathcal{V}$ outputs (cd.read.end, sid, $\operatorname{com}_{i}, \operatorname{com}_{r}$ ).

Fig. 2. Construction $\Pi_{\mathrm{CD}}$ : interfaces cd.setup and cd.read

A VC Scheme From the DHE Assumption. We show a VC scheme that is secure under the Diffie-Hellman Exponent (DHE) assumption [6]. Let $k \in \mathbb{N}$ denote the security parameter and let $\epsilon(k)$ denote a negligible function. We recall the $\ell$-DHE assumption.

Definition 6.1: [ $\ell$-DHE] Let $\left(p, \mathbb{G}, \widetilde{\mathbb{G}}, \mathbb{G}_{t}, e, g, \tilde{g}\right) \leftarrow \mathcal{G}\left(1^{k}\right)$ and $\alpha \leftarrow \mathbb{Z}_{p}$. Given $\left(p, \mathbb{G}, \tilde{\mathbb{G}}, \mathbb{G}_{t}, e, g, \tilde{g}\right)$ and a tuple $\left(g_{1}, \tilde{g}_{1}\right.$, $\left.\ldots, g_{\ell}, \tilde{g}_{\ell}, g_{\ell+2}, \ldots, g_{2 \ell}\right)$ such that $g_{i}=g^{\left(\alpha^{i}\right)}$ and $\tilde{g}_{i}=\tilde{g}^{\left(\alpha^{i}\right)}$, for any p.p.t. adversary $\mathcal{A}, \operatorname{Pr}\left[g^{\left(\alpha^{\ell+1}\right)} \leftarrow \mathcal{A}\left(p, \mathbb{G}, \widetilde{\mathbb{G}}, \mathbb{G}_{t}, e, g\right.\right.$, $\left.\left.\tilde{g}, g_{1}, \tilde{g}_{1}, \ldots, g_{\ell}, \tilde{g}_{\ell}, g_{\ell+2}, \ldots, g_{2 \ell}\right)\right] \leq \epsilon(k)$.

VC.Setup $\left(1^{k}, \ell\right)$. Generate groups $\left(p, \mathbb{G}, \widetilde{\mathbb{G}}, \mathbb{G}_{t}, e, g, \tilde{g}\right) \leftarrow$ $\mathcal{G}\left(1^{k}\right)$, pick $\alpha \leftarrow \mathbb{Z}_{p}$, and compute $\left(g_{1}, \tilde{g}_{1}, \ldots, g_{\ell}, \tilde{g}_{\ell}\right.$, $\left.g_{\ell+2}, \ldots, g_{2 \ell}\right)$, where $g_{i}=g^{\left(\alpha^{i}\right)}$ and $\tilde{g}_{i}=\tilde{g}^{\left(\alpha^{i}\right)}$. Output the parameters par $=\left(p, \mathbb{G}, \tilde{\mathbb{G}}, \mathbb{G}_{t}, e, g, \tilde{g}, g_{1}, \tilde{g}_{1}, \ldots, g_{\ell}\right.$, $\left.\tilde{g}_{\ell}, g_{\ell+2}, \ldots, g_{2 \ell}, \mathcal{M}=\mathbb{Z}_{p}, \mathcal{R}=\mathbb{Z}_{p}\right)$.

VC.Commit $(p a r, \mathbf{x}, r)$. Let $|\mathbf{x}|=n \leq \ell$. Output

$$
v c=g^{r} \cdot \prod_{j=1}^{n} g_{\ell+1-j}^{\mathbf{x}[j]}=g^{r} \cdot g_{\ell}^{\mathbf{x}[1]} \cdots g_{\ell+1-n}^{\mathbf{x}[n]} .
$$

VC.Wit $(p a r, i, \mathbf{x}, r)$. Let $|\mathbf{x}|=n \leq \ell$. Output

$$
w=g_{i}^{r} \cdot \prod_{j=1, j \neq i}^{n} g_{\ell+1-j+i}^{\mathbf{x}[j]} .
$$




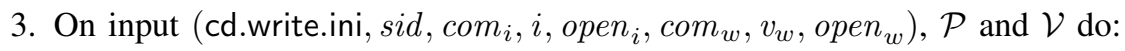

- $\mathcal{P}$ takes the stored tuple (sid, cp, par, vc, $\mathbf{x}, r)$.

- $\mathcal{P}$ parses com $_{i}$ as $\left(\right.$ com $_{i}^{\prime}$, parcom $_{i}$, COM.Verify $\left._{i}\right)$.

- $\mathcal{P}$ parses $\operatorname{com}_{w}$ as $\left(\operatorname{com}_{w}^{\prime}, \operatorname{parcom}_{w}\right.$, COM.Verify $\left.{ }_{w}\right)$.

- If $(s i d, i, w)$ is not stored, $\mathcal{P}$ computes a VC witness $w$ for position $i$ : run $w \leftarrow \operatorname{VC}$. Wit $($ par, $i, \mathbf{x}, r)$ and store (sid, $i, w)$.

- $\mathcal{P}$ updates the vector commitment $v c$ to $v c^{\prime}$ : pick random $r^{\prime} \leftarrow \mathcal{R}$ and run $v c^{\prime} \leftarrow$ VC.ComUpd $\left(p a r, v c, i, v_{r}, r\right.$, $\left.v_{w}, r^{\prime}\right)$, where $v_{r} \leftarrow \mathbf{x}[i]$.

- $\mathcal{P}$ increments the counter of write operations started $c p^{\prime} \leftarrow c p+1$.

- $\mathcal{P}$ sets wit $_{w} \leftarrow\left(w, i\right.$, open $_{i}, v_{r}, v_{w}$, open $\left._{w}, r, r^{\prime}\right)$.

- $\mathcal{P}$ sets $i n s_{w} \leftarrow\left(\right.$ par, vc, vc', parcom $\left._{i}, \operatorname{com}_{i}^{\prime}, \operatorname{parcom}_{w}, \operatorname{com}_{w}^{\prime}, c p^{\prime}\right)$.

- $\mathcal{P}$ updates the vector $\mathbf{x}$ to $\mathbf{x}^{\prime}$ : set $\mathbf{x}^{\prime} \leftarrow \mathbf{x}$ and $\mathbf{x}^{\prime}[i] \leftarrow v_{w}$.

- $\mathcal{P}$ updates the stored tuple to (sid, $c p^{\prime}$, par, $\left.v c^{\prime}, \mathbf{x}^{\prime}, r^{\prime}\right)$.

- $\mathcal{P}$ updates the stored witnesses: for $j=1$ to $N_{\text {max }}$, if $(\operatorname{sid}, j, w)$ is stored, run $w^{\prime} \leftarrow \operatorname{VC}$.WitUpd $(p a r, w, j, i, x, r$, $\left.x^{\prime}, r^{\prime}\right)$ and update to $\left(\right.$ sid, $\left.j, w^{\prime}\right)$.

- $\mathcal{P}$ uses the zk.prove interface to send wit $_{w}$ and $i n s_{w}$ to $\mathcal{F}_{\mathrm{ZK}}^{R_{w}}$, where $R_{w}$ is

$$
\begin{aligned}
R_{w}= & \left\{\left(\text { wit }_{w}, \text { ins }_{w}\right):\right. \\
& 1=\text { COM.Verify }_{i}\left(\text { parcom }_{i}, \text { com }_{i}^{\prime}, i, \text { open }_{i}\right) \wedge \\
& 1=\text { COM.Verify }_{w}\left(\text { parcom }_{w}, \text { com }_{w}^{\prime}, v_{w}, \text { open }_{w}\right) \wedge \\
& \left.1=\text { VC.VerComUpd }\left(\text { par }, v c, v c^{\prime}, w, i, v_{r}, r, v_{w}, r^{\prime}\right)\right\}
\end{aligned}
$$

In equation 4 and equation 5 , the prover proves that $\operatorname{com}_{i}^{\prime}$ and $\operatorname{com}_{w}^{\prime}$ are commitments to $i$ and $v_{w}$ respectively. In equation 6 , the prover proves that $v_{r}$ is stored in the position $i$ in the vector commitment $v c$, and that $v c^{\prime}$ is a vector commitment that stores the same values as $v c$, except that it stores $v_{w}$ in the position $i$ and that its random value is $r^{\prime}$ instead of $r$.

- $\mathcal{V}$ gets ins $_{w}=\left(p \hat{a} r, \hat{v c}, v c^{\prime}, \operatorname{parcom}_{i}, \operatorname{com}_{i}^{\prime}, \operatorname{parcom}_{w}, \operatorname{com}_{w}^{\prime}, c p\right)$.

- $\mathcal{V}$ takes the stored tuple (sid, cv, par, vc).

- $\mathcal{V}$ aborts if $c p \neq c v+1$, or if $p \hat{a} r \neq p a r$, or if $\hat{v c} \neq v c$.

- $\mathcal{V}$ sets $c v^{\prime} \leftarrow c v+1$ and updates the stored tuple to (sid, $\left.c v^{\prime}, p a r, v c^{\prime}\right)$.

- $\mathcal{V}$ sets com $_{i} \leftarrow\left(\right.$ com $_{i}^{\prime}$, parcom $_{i}$, COM.Verify $\left._{i}\right)$.

- $\mathcal{V}$ sets $\operatorname{com}_{w} \leftarrow\left(\operatorname{com}_{w}^{\prime}, \operatorname{parcom}_{w}\right.$, COM.Verify $\left._{w}\right)$.

- $\mathcal{V}$ outputs (cd.write.end, sid, $\left.\operatorname{com}_{i}, \operatorname{com}_{w}\right)$.

Fig. 3. Construction $\Pi_{\mathrm{CD}}$ : interface cd.write

VC. $\operatorname{Verify}(p a r, v c, x, i, w)$. Output 1 if $e\left(v c, \tilde{g}_{i}\right)=e(w, \tilde{g})$. $e\left(g_{1}, \tilde{g}_{\ell}\right)^{x}$, else output 0 .

VC.ComUpd $\left(p a r, v c, j, x, r, x^{\prime}, r^{\prime}\right)$. Output the commitment

$$
v c^{\prime}=v c \cdot \frac{g^{r^{\prime}} \cdot g_{\ell+1-j}^{x^{\prime}}}{g^{r} \cdot g_{\ell+1-j}^{x}}=v c \cdot g^{r^{\prime}-r} \cdot g_{\ell+1-j}^{x^{\prime}-x} .
$$

VC.VerComUpd $\left(p a r, v c, v c^{\prime}, w, j, x, r, x^{\prime}, r^{\prime}\right)$. Compute $v \leftarrow$ VC. Verify $($ par, $v c, x, j, w)$. If $v \leftarrow 0$, output $v$, else run $\overline{v c} \leftarrow \operatorname{VC}$.ComUpd $\left(p a r, v c, j, x, r, x^{\prime}, r^{\prime}\right)$. If $\overline{v c}=v c^{\prime}$, output 1 , else output 0 .

VC.WitUpd $\left(p a r, w, i, j, x, r, x^{\prime}, r^{\prime}\right)$. If $i=j$, output $w$. Otherwise output the witness

$$
w^{\prime}=w \cdot \frac{g_{i}^{r^{\prime}} \cdot g_{\ell+1-j+i}^{x^{\prime}}}{g_{i}^{r} \cdot g_{\ell+1-j+i}^{x}}=w \cdot g_{i}^{r^{\prime}-r} \cdot g_{\ell+1-j+i}^{x^{\prime}-x} .
$$

Theorem 6.2: This VC scheme is correct, hiding, and binding under the $\ell$-DHE assumption.
We prove this theorem in Appendix $\mathbf{J}$.

Notation for ZK Proofs of Knowledge. We use classical results for efficient $\mathrm{ZK}$ proofs of knowledge for discrete logarithm relations. In the notation of [25], a UC ZK protocol proving knowledge of exponents $\left(w_{1}, \ldots, w_{n}\right)$ satisfying the formula $\phi\left(w_{1}, \ldots, w_{n}\right)$ is described as

$$
\lambda w_{1}, \ldots, w_{n}: \phi\left(w_{1}, \ldots, w_{n}\right)
$$

The formula $\phi\left(w_{1}, \ldots, w_{n}\right)$ consists of conjunctions and disjunctions of "atoms". An atom expresses group relations, such as $\prod_{j=1}^{k} g_{j}^{\mathcal{F}_{j}}=1$, where the $g_{j}$ 's are elements of prime order groups and the $\mathcal{F}_{j}$ 's are polynomials in the variables $\left(w_{1}, \ldots, w_{n}\right)$.

A proof system for (7) can be transformed into a proof system for the following more expressive statements about secret exponents sexps and secret bases sbases:

$$
\text { 入 sexps, sbases : } \phi(\text { sexps, bases } \cup \text { sbases })
$$


The transformation adds an additional base $h$ to the public bases. For each $g_{j} \in$ sbases, the transformation picks a random exponent $\rho_{j}$ and computes a blinded base $g_{j}^{\prime}=g_{j} h^{\rho_{j}}$. The transformation adds $g_{j}^{\prime}$ to the public bases bases, $\rho_{j}$ to the secret exponents sexps, and rewrites $g_{j}^{\mathcal{F}_{j}}$ into $g_{j}^{\prime \mathcal{F}_{j}} h^{-\mathcal{F}_{j} \rho_{j}}$.

The proof system supports pairing product equations $\prod_{j=1}^{k} e\left(g_{j}, \tilde{g}_{j}\right)^{\mathcal{F}_{j}}=1$ in groups of prime order with a bilinear map $e$, by treating the target group $\mathbb{G}_{t}$ as the group of the proof system. The embedding for secret bases is unchanged, except for the case in which both bases in a pairing are secret. In the latter case, $e\left(g_{j}, \tilde{g}_{j}\right)^{\mathcal{F}_{j}}$ needs to be transformed into $e\left(g_{j}^{\prime}, \tilde{g}_{j}^{\prime}\right)^{\mathcal{F}_{j}} e\left(g_{j}^{\prime}, \tilde{h}\right)^{-\mathcal{F}_{j} \tilde{\rho}_{j}} e\left(h, \tilde{g}_{j}^{\prime}\right)^{-\mathcal{F}_{j} \rho_{j}} e(h, \tilde{h})^{\mathcal{F}_{j} \rho_{j} \tilde{\rho}_{j}}$.

Structure-Preserving Signatures. A signature scheme consists of the algorithms KeyGen, Sign and VfSig. Algorithm KeyGen $\left(1^{k}\right)$ outputs a secret key $s k$ and a public key $p k$, which include a description of the message space $\mathcal{M}$. Sign $(s k, m)$ outputs a signature $s$ on the message $m \in \mathcal{M}$. VfSig $(p k, s, m)$ outputs 1 if $s$ is a valid signature on $m$ and 0 otherwise. This definition can be extended to blocks of messages $\bar{m}=\left(m_{1}\right.$, $\left.\ldots, m_{n}\right)$. In this case, $\operatorname{KeyGen}\left(1^{k}, n\right)$ receives the maximum number of messages as input. A signature scheme must fulfill the correctness and existential unforgeability properties [26].

In structure-preserving signatures (SPS), the public key, the messages, and the signatures are group elements in $\mathbb{G}$ and $\tilde{\mathbb{G}}$, and verification must consist purely in the checking of pairing product equations. We employ SPS to sign group elements, while still supporting efficient $\mathrm{ZK}$ proofs of signature possession. For concreteness, we recall the scheme proposed by [27] for the case in which $a$ elements in $\mathbb{G}$ and $b$ elements in $\tilde{\mathbb{G}}$ are signed. This scheme is strongly existentially unforgeable against adaptive chosen message attacks in the generic group model [27].

$\operatorname{KeyGen}(g r p, a, b)$. Let $g r p \leftarrow\left(p, \mathbb{G}, \tilde{\mathbb{G}}, \mathbb{G}_{t}, e, g, \tilde{g}\right)$ be the bilinear map parameters. Pick at random $u_{1}, \ldots, u_{b}, v$, $w_{1}, \ldots w_{a}, z \leftarrow \mathbb{Z}_{p}^{*}$ and compute $U_{i}=g^{u_{i}}, i \in[1 . . b]$, $V=\tilde{g}^{v}, W_{i}=\tilde{g}^{w_{i}}, i \in[1 . . a]$ and $Z=\tilde{g}^{z}$. Return the verification key $p k \leftarrow\left(g r p, U_{1}, \ldots, U_{b}, V, W_{1}, \ldots, W_{a}\right.$, $Z)$ and the signing key $s k \leftarrow\left(p k, u_{1}, \ldots, u_{b}, v, w_{1}, \ldots\right.$, $\left.w_{a}, z\right)$.

$\operatorname{Sign}\left(s k,\left\langle m_{1}, \ldots, m_{a+b}\right\rangle\right)$. Pick $r \leftarrow \mathbb{Z}_{p}^{*}$, compute $R \leftarrow g^{r}$, $S \leftarrow g^{z-r v} \prod_{i=1}^{a} m_{i}^{-w_{i}}$, and $T \leftarrow\left(\tilde{g} \prod_{i=1}^{b} m_{a+i}^{-u_{i}}\right)^{1 / r}$, and output the signature $s \leftarrow(R, S, T)$.

$\operatorname{VfSig}\left(p k, s,\left\langle m_{1}, \ldots, m_{a+b}\right\rangle\right)$. Output 1 if it holds both that $e(R, V) e(S, \tilde{g}) \prod_{i=1}^{a} e\left(m_{i}, W_{i}\right)=e(g, Z)$ and $e(R, T)$ $\prod_{i=1}^{b} e\left(U_{i}, m_{a+i}\right)=e(g, \tilde{g})$.

Commitment Schemes. A commitment scheme consists of algorithms CSetup, Com and VfCom. The algorithm CSetup $\left(1^{k}\right)$ generates the parameters of the commitment scheme $\operatorname{par}_{c}$, which include a description of the message space $\mathcal{M}$. Com $\left(\operatorname{par}_{c}, x\right)$ outputs a commitment $\operatorname{com}$ to $x \in \mathcal{M}$ and some auxiliary information open. The verification algorithm $\mathrm{VfCom}\left(\operatorname{par}_{c}\right.$, com,$x$, open $)$ outputs 1 if com is a commitment to $x \in \mathcal{M}$ with some auxiliary information open or 0 if that is not the case. A commitment scheme should fulfill the correctness, trapdoor and binding properties as described in [5].
The Pedersen commitment scheme [28] fulfills the correctness, trapdoor and binding properties. In [5], it is proven that any commitment scheme that fulfills those properties fulfills the ideal functionality $\mathcal{F}_{\text {NIC }}$. The Pedersen commitment scheme works as follows. CSetup $\left(1^{k}\right)$ takes a group $\mathbb{G}$ of prime order $p$ with generator $g$, picks random $\alpha$, computes $h \leftarrow g^{\alpha}$ and sets the parameters $\operatorname{par}_{c} \leftarrow(\mathbb{G}, g, h)$, which include a description of the message space $\mathcal{M} \leftarrow \mathbb{Z}_{p}$. Com $\left(\operatorname{par}_{c}, x\right)$ picks random open $\leftarrow \mathbb{Z}_{p}$ and outputs a commitment com $\leftarrow g^{x} h^{\text {open }}$ to $x \in \mathcal{M}$ and some auxiliary information open. The algorithm $\operatorname{VfCom}\left(\right.$ par $_{c}$, com, $x$, open $)$ outputs 1 if com $=g^{x} h^{\text {open }}$.

\section{B. ZK Proofs for $R_{r}$ and $R_{w}$}

In $\Pi_{\mathrm{CD}}$, we need to compute two $\mathrm{ZK}$ proofs of knowledge: a proof for a read operation and a proof for a write operation. We describe these proofs for the $\ell$-DHE VC scheme.

For a read operation, we need a $\mathrm{ZK}$ proof of knowledge of a witness $w$ that a value $v_{r}$ was committed to in a vector commitment $v c$ at position $i$. I.e., we need to compute a proof

$$
\begin{aligned}
& \boldsymbol{X}_{i}, \text { open }_{i}, v_{r}, \text { open }_{r}, w: \\
& 1=\text { COM.Verify }_{i}\left(\text { parcom }_{i}, \text { com }_{i}^{\prime}, i, \text { open }_{i}\right) \wedge \\
& 1=\text { COM.Verify }_{r}\left(\text { parcom }_{r}, \text { com }_{r}^{\prime}, v_{r}, \text { open }_{r}\right) \wedge \\
& \left.1=\text { VC.Verify }\left(\text { par }, v c, v_{r}, i, w\right) .\right\}
\end{aligned}
$$

This proof involves proving knowledge of a position $i$, a value $v_{r}$ and a witness $w$ such that the verification equation $e\left(v c, \tilde{g}_{i}\right)$ $=e(w, g) e\left(g_{1}, \tilde{g}_{\ell}\right)^{v_{r}}$ holds. Additionally, it involves proving that the position $i$ is committed in a commitment $\operatorname{com}_{i}^{\prime}$ with opening open ${ }_{i}$, and the value $v_{r}$ is committed in a commitment com $_{r}^{\prime}$ with opening open .

Because $\alpha$ is secret, the relation between $\tilde{g}_{i}=\tilde{g}^{\alpha^{i}}$ and $i$ is not efficiently provable. For this reason, we extend the parameters of the VC scheme with structure preserving signatures (SPS) that bind $i$ with $\tilde{g}_{i}$. We also need to bind $i$ with $g_{\ell+1-i}$ for a ZK proof of a write operation. (In practice, $i$ does not necessarily need to belong to $\left[1, N_{\max }\right]$, i.e., other unique identifiers in $\mathbb{Z}_{p}$ can be assigned to the positions.) To this end, the setup algorithm of the VC scheme is extended as follows.

VC.Setup $\left(1^{k}, \ell\right)$. Generate groups $\left(p, \mathbb{G}, \tilde{\mathbb{G}}, \mathbb{G}_{t}, e, g, \tilde{g}\right) \leftarrow$ $\mathcal{G}\left(1^{k}\right)$, pick $\alpha \leftarrow \mathbb{Z}_{p}$, and compute $\left(g_{1}, \tilde{g}_{1}, \ldots, g_{\ell}\right.$, $\left.\tilde{g}_{\ell}, g_{\ell+2}, \ldots, g_{2 \ell}\right)$, where $g_{i}=g^{\left(\alpha^{i}\right)}$ and $\tilde{g}_{i}=\tilde{g}^{\left(\alpha^{i}\right)}$. Compute $(s k, p k) \leftarrow \operatorname{KeyGen}(g r p, 1,2)$. For $i \in[1, \ell]$, run $s_{i} \leftarrow \operatorname{Sign}\left(s k,\left\langle g_{\ell+1-i}, \tilde{g}_{i}, \tilde{g}^{i}\right\rangle\right)$. Compute additional bases $h \leftarrow \mathbb{G}$ and $\tilde{h} \leftarrow \tilde{\mathbb{G}}$. Output the parameters par $=\left(p, \mathbb{G}, \tilde{\mathbb{G}}, \mathbb{G}_{t}, e, g, \tilde{g}, g_{1}, \tilde{g}_{1}, \ldots, g_{\ell}, \tilde{g}_{\ell}, g_{\ell+2}, \ldots\right.$, $\left.g_{2 \ell}, p k, s_{1}, \ldots, s_{\ell}, h, \tilde{h}, \mathcal{M}=\mathbb{Z}_{p}, \mathcal{R}=\mathbb{Z}_{p}\right)$.

Therefore, our instantiation of $\Pi_{\mathrm{CD}}$ is secure under the unforgeability property of the SPS scheme in addition to the $\ell$ DHE assumption. Let $(g, h)$ be the parameters of the Pedersen commitment scheme for both parcom $_{i}$ and parcom $_{r}$. Let $\left(U_{1}, U_{2}, V, W_{1}, Z\right)$ be the public key of the signature scheme. 
Let $(R, S, T)$ be a signature on $\left(g_{\ell+1-i}, \tilde{g}_{i}, \tilde{g}^{i}\right)$. We describe the proof as follows.

$$
\begin{aligned}
& \lambda i, \text { open }_{i}, v_{r}, \text { open }_{r}, \tilde{g}_{i}, g_{\ell+1-i}, w, R, S, T: \\
& \operatorname{com}_{i}^{\prime}=g^{i} h^{\text {open }_{i}} \wedge \\
& \operatorname{com}_{r}^{\prime}=g^{v_{r}} h^{\text {open }_{r}} \wedge \\
& \quad e(R, V) e(S, \tilde{g}) e\left(g_{\ell+1-i}, W_{1}\right) e(g, Z)^{-1}=1 \wedge \\
& \quad e(R, T) e\left(U_{1}, \tilde{g}_{i}\right) e\left(U_{2}, \tilde{g}\right)^{i} e(g, \tilde{g})^{-1}=1 \wedge \\
& \quad e\left(v c, \tilde{g}_{i}\right)^{-1} e(w, \tilde{g}) e\left(g_{1}, \tilde{g}_{\ell}\right)^{v_{r}}=1
\end{aligned}
$$

Equation 9 and Equation 10 prove knowledge of the openings of the Pedersen commitments $\mathrm{com}_{i}^{\prime}$ and $\mathrm{com}_{r}^{\prime}$. Equation 11 and Equation 12 prove knowledge of a signature $(R, S, T)$ on a message $\left(g_{\ell+1-i}, \tilde{g}_{i}, \tilde{g}^{i}\right)$. Equation 13 proves that the value $v_{r}$ in $c o m_{r}^{\prime}$ is equal to the value committed in the position $i$ of the vector commitment $v c$. To prove knowledge of the secret bases $\left(\tilde{g}_{i}, g_{\ell+1-i}, w, R, S, T\right)$, the equations need to be modified as described in Section VI-A.

For a write operation, we need a ZK proof of knowledge that a vector commitment $v c^{\prime}$ is an update of a vector commitment $v c$ that contains randomness $r^{\prime}$ instead of $r$ and value $v_{w}$ instead of $v_{r}$ at a position $i$.

$$
\begin{aligned}
& \rtimes i, \text { open }_{i}, v_{w}, \text { open }_{w}, v_{r}, r, r^{\prime}, w: \\
& 1=\operatorname{VfCom}\left(\text { parcom }_{i}, \text { com }_{i}^{\prime}, i, \text { open }_{i}\right) \wedge \\
& 1=\operatorname{VfCom}\left(\text { parcom }_{w}, \operatorname{com}_{w}^{\prime}, v_{w}, \text { open }_{w}\right) \wedge \\
& 1=\operatorname{VC} \cdot \operatorname{VerComUpd}\left(\text { par }, v c, v c^{\prime}, w, i, v_{r}, r, v_{w}, r^{\prime}\right)
\end{aligned}
$$

Let $(g, h)$ be the parameters of the Pedersen commitment scheme for both parcom $_{i}$ and parcom $_{w}$. Let $\left(U_{1}, U_{2}, V, W_{1}\right.$, $Z)$ be the public key of the signature scheme. Let $(R, S, T)$ be a signature on $\left(g_{\ell+1-i}, \tilde{g}_{i}, \tilde{g}^{i}\right)$. We describe the proof as follows.

$$
\begin{aligned}
& \lambda i, \text { open }_{i}, v_{w}, \text { open }_{w}, v_{r}, r^{\prime}-r, \tilde{g}_{i}, g_{\ell+1-i}, w, R, S, T: \\
& \operatorname{com}_{i}^{\prime}=g^{i} h^{\text {open }_{i}} \wedge \\
& \operatorname{com}_{w}^{\prime}=g^{v_{w}} h^{\text {open }_{w}} \wedge \\
& \quad e(R, V) e(S, \tilde{g}) e\left(g_{\ell+1-i}, W_{1}\right) e(g, Z)^{-1}=1 \wedge \\
& e(R, T) e\left(U_{1}, \tilde{g}_{i}\right) e\left(U_{2}, \tilde{g}\right)^{i} e(g, \tilde{g})^{-1}=1 \wedge \\
& e\left(v c, \tilde{g}_{i}\right)^{-1} e(w, \tilde{g}) e\left(g_{1}, \tilde{g}_{\ell}\right)^{v_{r}}=1 \wedge \\
& v c^{\prime} / v c=g^{r^{\prime}-r} \cdot g_{\ell+1-i}^{v_{w}-v_{r}}
\end{aligned}
$$

Equation 14 and Equation 15 prove knowledge of the openings of the Pedersen commitments $\operatorname{com}_{i}^{\prime}$ and $\operatorname{com}_{w}^{\prime}$. Equation 16 and Equation 17 prove knowledge of a signature $(R, S, T)$ on a message $\left(g_{\ell+1-i}, \tilde{g}_{i}, \tilde{g}^{i}\right)$. Equation 18 proves that $v_{r}$ is the value committed in the position $i$ of the vector commitment $v c$. Equation 19 proves that $v c^{\prime}$ is an update of $v c$ that contains $v_{w}$ instead of $v_{r}$ at position $i$. To prove knowledge of the secret bases $\left(\tilde{g}_{i}, g_{\ell+1-i}, w, R, S, T\right)$, the equations need to be modified as described in Section VI-A.

\section{Efficiency Analysis}

We analyze the storage, communication, and computation costs of our instantiation of $\Pi_{\mathrm{CD}}$. We summarize them in Table I.
Storage Cost. $\mathcal{P}$ stores the common reference string, which consists of the parameters of the VC scheme. Its size grows linearly with the maximum size $N_{\max }$ of the database. Throughout the protocol execution, in addition to the common reference string, $\mathcal{P}$ also stores the last update of the vector commitment, the committed vector, the randomness used to compute that commitment, and the witnesses computed so far. In conclusion, the storage cost for $\mathcal{P}$ grows linearly with $N_{\max }$.

$\mathcal{V}$ also stores the common reference string. Although in $\Pi_{\mathrm{CD}}$, $\mathcal{V}$ stores the whole common reference string, we observe that in our instantiation of $\Pi_{\mathrm{CD}}$ this is not necessary. In practice, $\mathcal{V}$ only needs to store $\left(g, \tilde{g}, h, \tilde{h}, g_{1}, \tilde{g}_{\ell}\right)$ and the public key $\left(U_{1}\right.$, $\left.U_{2}, V, W_{1}, Z\right)$ of the signature scheme. These values suffice to verify the ZK proofs of knowledge for read and write operations. In addition to the common reference string, $\mathcal{V}$ only needs to store the last update of the vector commitment. Therefore, the storage cost for $\mathcal{V}$ is constant and independent of $N_{\max }$.

Communication Cost. In the setup phase, the communication grows with $N_{\max }$ because $\mathcal{V}$ sends $\mathrm{Tbl}_{c d}$ to $\mathcal{P}$. This step can be avoided in practice if $\mathrm{Tbl}_{c d}$ is initialized to default values known by $\mathcal{P}$ and $\mathcal{V}$. In a read operation, $\mathcal{P}$ and $\mathcal{V}$ run a ZK proof of knowledge for $R_{r}$. The size of the witness and of the instance is constant and independent of $N_{\max }$. Therefore, the communication cost of the proof is independent of $N_{\text {max }}$. Similarly, in a write operation, $\mathcal{P}$ sends a ZK proof of knowledge for $R_{w}$ to $\mathcal{V}$. The size of the witness and of the instance is also independent of $N_{\max }$, and thus the communication cost of the proof is independent of $N_{\max }$.

Computation Cost. To compute a proof for a read operation, $\mathcal{P}$ first needs to compute a $\mathrm{VC}$ witness for the position to be read. If a witness $w$ was not computed before, the computation cost of this step grows linearly with $N_{\max }$. When a witness has already been computed, the computation cost is independent of $N_{\max }$. Concretely, if the database was not updated since the moment $w$ was computed, the same witness $w$ can be reused. If the database was updated, $w$ can be updated with a computation cost linear in the number of updates, but independent of $N_{\max }$.

The remaining steps in the computation of the proof for $R_{r}$ are also independent of $N_{\max }$. Therefore, after computing a witness $w$ for a position, the remaining proofs can be computed with cost independent of $N_{\max }$.

A proof for a write operation also requires the computation of a witness $w$ for the position to be written. The same optimization used for a read proof can be applied here, i.e., if a witness for that position has already been computed, a new witness can be computed with cost independent of $N_{\max }$. In addition to the witness, $\mathcal{P}$ also needs to update the vector commitment. The computation cost of a vector commitment update is also independent of $N_{\max }$. The remaining steps to compute the proof for $R_{w}$ are also independent of $N_{\max }$.

Worst/Average/Best Case Computation Cost. The computation cost depends on the number of positions that $\mathcal{P}$ needs to read or write throughout the protocol execution, as well as on the number of values in the database that are 0 .

In the worst case, $\mathcal{P}$ needs to read and/or write all the positions in the database throughout the protocol execution. In 
TABLE I

EFFICIENCY ANALYSIS OF OUR CONSTRUCTION.

\begin{tabular}{|l|c|c|}
\hline & Prover & Verifier \\
\hline Param Size & $(4 \ell+4)|\mathbb{G}|+(2 \ell+5)|\tilde{\mathbb{G}}|$ & $5|\mathbb{G}|+6|\tilde{\mathbb{G}}|$ \\
\hline \hline \multicolumn{2}{|c|}{ Communication Cost } \\
\hline Setup (default Tbl Tbd $\left._{c d}\right)$ & const. & const. \\
\hline Setup $\left(\mathcal{V}\right.$ sends Tbl $\left._{c d}\right)$ & $N_{\max }$ & $N_{\max }$ \\
\hline Read & const. & const. \\
\hline Write & const. & const. \\
\hline \hline \multicolumn{2}{|c|}{ Computation Cost } \\
\hline Setup $(v c$ computation) & $N_{\max }$ & $N_{\max }$ \\
\hline R/W: $w$ not computed & $N_{\max }$ & const. \\
\hline R/W: $w$ not updated & $n_{u p d}$ & const. \\
\hline R/W: $w$ updated & const. & const. \\
\hline
\end{tabular}

this case, $\mathcal{P}$ computes $v c$ with cost linear in $\mathbb{Z}_{p}$, and computes $N_{\max }$ VC witnesses $w$ with a total cost that grows quadratically with $N_{\max }$. Furthermore, if the values stored in the database are big (e.g. random values in $\mathbb{Z}_{p}$ ), the computation cost of $v c$ and each $w$ is negatively affected.

In the best case, $\mathcal{P}$ needs to read and/or write a small subset of positions, and the database is initialized to a vector of zeroes. In this case, the computation cost for $v c$ is constant (because the database is initialized to zeroes), and $\mathcal{P}$ only needs to compute $\mathrm{VC}$ witnesses $w$ for those positions that are $\mathrm{read} /$ written. Moreover, the cost of computing those witnesses only increases linearly with the number of non-zero values stored in the database (instead of linearly with $N_{\max }$ ). Because only a small subset of positions are written, most values in the database are 0 . Furthermore, if the values written into the database are small numbers (rather than big or random numbers in $\mathbb{Z}_{p}$ ), the computation of each VC witness $w$ is very efficient.

The average case would be somewhere in between the worst and best cases. I.e., $\mathcal{P}$ reads and/or writes a relatively small number of positions, and most of the values in the database are 0 or relatively small.

Efficiency measurements. Let $|\mathbb{G}|,|\tilde{\mathbb{G}}|$, and $\left|\mathbb{G}_{t}\right|$ be the bit length of $\mathbb{G}, \widetilde{\mathbb{G}}$, and $\mathbb{G}_{t}$, respectively. In the DHE VC scheme, given the maximum vector length $\ell$, the parameters are of size $(4 \ell+4) \cdot|\mathbb{G}|+(2 \ell+5) \cdot|\tilde{\mathbb{G}}|$. (This includes the signatures needed for the proofs for relations $R_{r}$ and $R_{w}$.) We recall that $\mathcal{V}$ only needs to store a small part of the parameters, whose length is $5 \cdot|\mathbb{G}|+6 \cdot|\tilde{\mathbb{G}}|$. A vector commitment and a witness are of length $1 \cdot|\mathbb{G}|$. The cost of computing a vector commitment or a witness increases with $N_{\max }$, but the cost of updating commitments and witnesses increases only with the number of updated elements.

To compute the UC ZK proofs of knowledge for $R_{r}$ and $R_{w}$, we use the compiler in [25]. The public parameters of the proof system contain a public key of the Paillier encryption scheme, the parameters for a multi-integer commitment scheme and the specification of a DSA group. The cost of a proof depends on the number of secret elements in the witness, which is 10 in $R_{r}$ and 12 in $R_{w}$, and of the number of equations composed by Boolean ANDs, which is 5 in $R_{r}$ and 6 in $R_{w}$. The computation cost for $\mathcal{P}$ of a $\Sigma$-protocol for $R_{r}$ or for $R_{w}$ involves one evaluation of each of the equations and one multiplication per secret value in the witness. The compiler in [25] extends a $\Sigma$-protocol and requires, additionally, a computation of a multi-integer commitment that commits to the secret values in the witness, an evaluation of a Paillier encryption for each of the secret values in the witness, a $\Sigma$-protocol to prove that the commitment and the encryptions are correctly generated, and 3 exponentiations in the DSA group. The computation cost for $\mathcal{V}$, as well as the communication cost, also depends on the number of secret values in the witness and on the number of equations. Therefore, as the number of secret values in the witness and of equations is constant in our proofs for $R_{r}$ and $R_{w}$, the computation and communication cost of our proofs do not depend on $N_{\max }$.

\section{Modular Design with $\mathcal{F}_{\mathrm{CD}}$}

We describe how $\mathcal{F}_{\mathrm{CD}}$ is used as building block together with $\mathcal{F}_{\mathrm{NIC}}, \mathcal{F}_{\mathrm{ZK}}^{R_{i}}$ and $\mathcal{F}_{\mathrm{ZK}}^{R_{v}}$ to describe a hybrid protocol. Consider as a simple example a protocol where the prover $\mathcal{P}$ writes a value $v$ at position $i$ into the database and later on proves statements about $i$ and $v$ to the verifier $\mathcal{V}$. $\mathcal{P}$ needs to hide $i$ and $v$ from $\mathcal{V}$ when $v$ is written into and when it is read from the database. The construction works as follows:

1) $\mathcal{V}$ runs the com.setup interface of $\mathcal{F}_{\mathrm{NIC}}$.

2) $\mathcal{V}$ uses the cd.setup interface of $\mathcal{F}_{\mathrm{CD}}$ to initialize $\mathrm{Tbl} \mathrm{c}_{c d}$, which is sent to $\mathcal{P}$.

3) $\mathcal{P}$ runs the com.setup interface of $\mathcal{F}_{\text {NIC }}$.

4) $\mathcal{P}$ uses the com.commit interface of $\mathcal{F}_{\text {NIC }}$ to get commitment and opening $\left(\right.$ com $_{i}$, open $\left._{i}\right)$ to the position $i$.

5) $\mathcal{P}$ uses the com.commit interface of $\mathcal{F}_{\text {NIC }}$ to get commitment and opening $\left(\right.$ com $_{w}$, open $\left._{w}\right)$ to the value $v$.

6) $\mathcal{P}$ uses the cd.write interface of $\mathcal{F}_{\mathrm{CD}}$ on input the tuple $\left(\right.$ com $_{i}, i$, open $_{i}$, com $_{w}, v$, open $\left._{w}\right)$ to write the entry $[i, v]$ into $\mathrm{Tbl}_{c d} . \mathcal{V}$ receives $\left(\mathrm{com}_{i}, \mathrm{com}_{w}\right)$. Thanks to the hiding property of the commitments computed by $\mathcal{F}_{\mathrm{NIC}}, \mathcal{V}$ is oblivious to the position and the value being written.

7) $\mathcal{V}$ uses the com.validate interface of $\mathcal{F}_{\text {NIC }}$ to validate that com $_{i}$ contains the parameters and verification algorithm used by $\mathcal{F}_{\text {NIC. }}$.

8) $\mathcal{V}$ uses the com.validate interface of $\mathcal{F}_{\text {NIC }}$ to validate $\operatorname{com}_{w}$.

9) When $\mathcal{P}$ wants to prove a statement about $i$ and $v, \mathcal{P}$ uses the com.commit interface of $\mathcal{F}_{\mathrm{NIC}}$ to get a fresh commitment and opening $\left(\operatorname{com}_{i}{ }^{\prime}\right.$ open $\left.{ }_{i}^{\prime}\right)$ to $i$.

10) $\mathcal{P}$ uses the com.commit interface of $\mathcal{F}_{\text {NIC }}$ to get a fresh commitment and opening $\left(\mathrm{com}_{r}\right.$, open $\left._{r}\right)$ to $v$.

11) $\mathcal{P}$ uses the cd.read interface of $\mathcal{F}_{\mathrm{CD}}$ on input $\left(\mathrm{com}_{i}^{\prime}, i\right.$, open $_{i}^{\prime}$, com $_{r}, v$, open $\left._{r}\right)$ to read the entry $[i, v]$ in $\mathrm{Tbl}_{c d}$. $\mathcal{V}$ receives $\left(\operatorname{com}_{i}^{\prime}, \operatorname{com}_{r}\right)$. Thanks to the hiding property of the commitments computed by $\mathcal{F}_{\mathrm{NIC}}$, the verifier is oblivious to the position and the value being read.

12) $\mathcal{V}$ uses the com.validate interface of $\mathcal{F}_{\text {NIC }}$ to validate $\mathrm{com}_{i}^{\prime}$.

13) $\mathcal{V}$ uses the com.validate interface of $\mathcal{F}_{\mathrm{NIC}}$ to validate $\mathrm{com}_{r}$. 


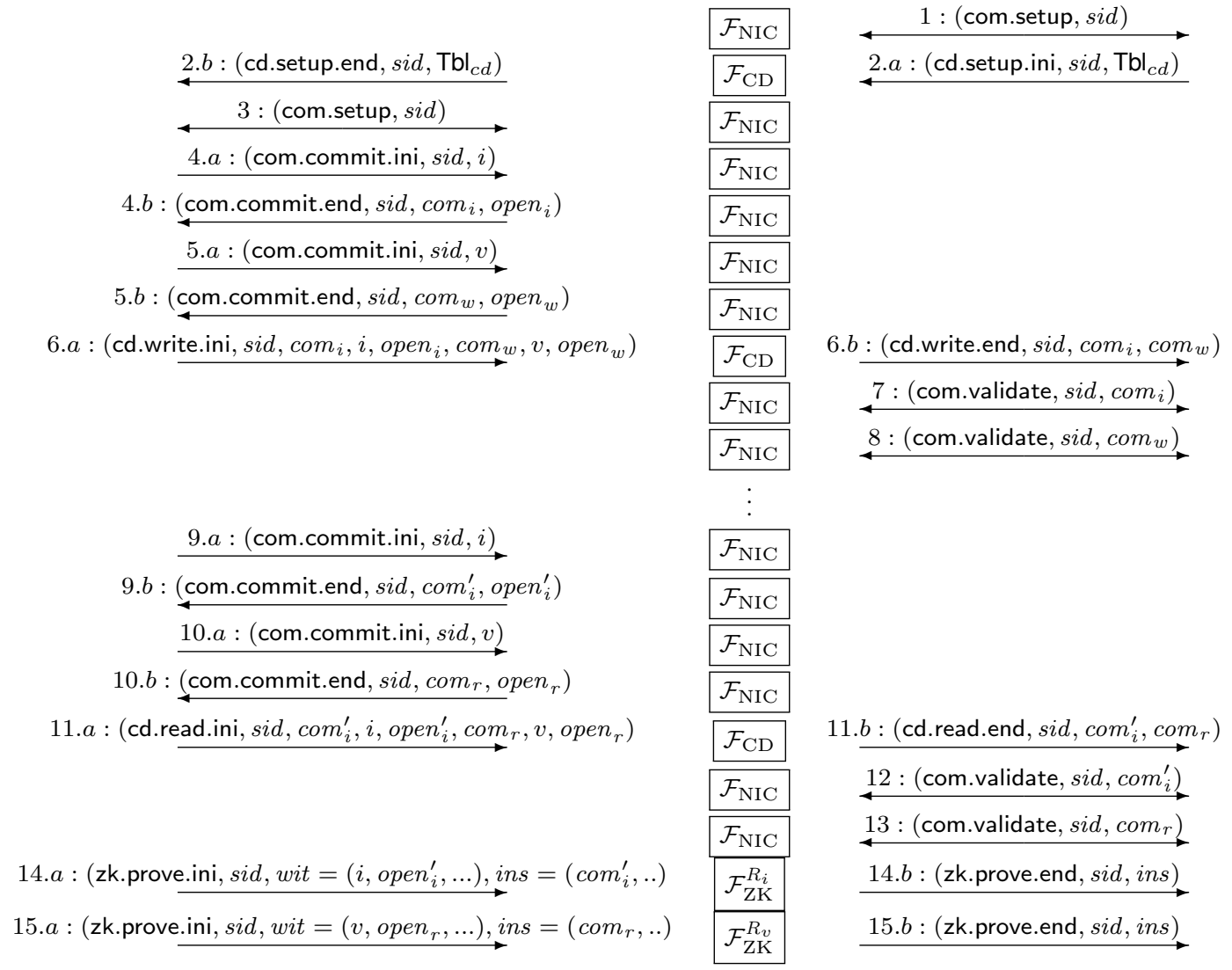

Fig. 4. High-level view on an example construction that uses $\mathcal{F}_{\mathrm{CD}}$ along with $\mathcal{F}_{\mathrm{NIC}}, \mathcal{F}_{\mathrm{ZK}}^{R_{i}}$ and $\mathcal{F}_{\mathrm{ZK}}^{R_{v}}$.

14) $\mathcal{P}$ uses the zk.prove interface of $\mathcal{F}_{\mathrm{ZK}}^{R_{i}}$ to prove statements about $i$. $\mathcal{P}$ sends an instance and a witness such that com $_{i}^{\prime}$ is in the instance, whereas $\left(i\right.$, open $\left._{i}^{\prime}\right)$ are in the witness. $\mathcal{V}$ receives the instance and checks that $\mathrm{com}_{i}^{\prime}$ in the instance is equal to the one received from $\mathcal{F}_{\mathrm{CD}}$. Then the binding property of the commitments computed by $\mathcal{F}_{\text {NIC }}$ guarantees that the same position $i$ that was input to $\mathcal{F}_{\mathrm{CD}}$ is now input to $\mathcal{F}_{\mathrm{ZK}}^{R_{i}}$.

15) Similarly, $\mathcal{P}$ uses the zk.prove interface of $\mathcal{F}_{\mathrm{ZK}}^{R_{v}}$ to prove statements about $v$.

This protocol, which we depict in Figure 4, hides from $\mathcal{V}$ that the commitments $\left(\mathrm{com}_{i}, \mathrm{com}_{w}\right)$ written into the database and the commitments $\left(\mathrm{com}_{i}^{\prime}, \mathrm{com}_{r}\right)$ read from the database commit to the same entry $[i, v]$. If this property is not needed, a simple protocol where $\mathcal{P}$ sends to $\mathcal{V}$ commitments to $i$ and $v$ and later on uses $\mathcal{F}_{\mathrm{ZK}}^{R_{i}}$ and $\mathcal{F}_{\mathrm{ZK}}^{R_{v}}$ to prove statements about $i$ and $v$ can be used. Therefore, $\mathcal{F}_{\mathrm{CD}}$ is particularly useful as building block of protocols where $\mathcal{P}$ needs to hide from $\mathcal{V}$ the positions read or written.

\section{APPLICATIONS OF $\mathcal{F}_{\mathrm{CD}}$}

$\mathcal{F}_{\mathrm{CD}}$ can be used as a building block in "commit-andprove" two-party protocols, where $\mathcal{P}$ commits to her inputs and subsequently proves in ZK statements about the committed values. Using $\mathcal{F}_{\mathrm{CD}}$ allows us to separate the task of storing values to be used in further ZK proofs from the task of proving statements in $\mathrm{ZK}$ about those values. Thanks to that, the design of the protocol is more modular, which leads to a simpler security analysis.

$\mathcal{F}_{\mathrm{CD}}$ is particularly appealing for protocols that need to hide the positions read or written from $\mathcal{V}$. A simple example of such a protocol is an OR proof. Our main application of $\mathcal{F}_{\mathrm{CD}}$ is its use as building block in protocols where $\mathcal{V}$ needs to obtain statistics about what $\mathcal{P}$ proves in zero-knowledge. To showcase this application, we describe a novel task we refer to as zero-knowledge counting, and we describe its application to privacy-preserving e-commerce and to privacy-preserving location sharing services. Moreover, we show that $\mathcal{F}_{\mathrm{CD}}$ can be used for gathering other types of statistics beyond zeroknowledge counting. Concretely, we discuss the application of $\mathcal{F}_{\mathrm{CD}}$ to privacy-preserving billing protocols. As described below, our applications of $\mathcal{F}_{\mathrm{CD}}$ to obtaining statistics are very efficient because they mirror the best case for computation cost described in VI-C.

OR proofs. In Appendix $\mathrm{K}$, we describe how $\mathcal{F}_{\mathrm{CD}}$ can be used to compute ZK proofs for OR relations, i.e., relations where $\mathcal{P}$ proves that at least one value in a database fulfills 
a statement, while hiding from $\mathcal{V}$ which of the values fulfills it. In a nutshell, the database of values for the OR relation is written into $\mathrm{Tbl}_{c d}$. After that, $\mathcal{P}$ simply reads one of the values from $\mathrm{Tbl}_{c d}$ and uses $\mathcal{F}_{\mathrm{ZK}}^{R}$ to prove in $\mathrm{ZK}$ that this value fulfills the statement. $\mathcal{F}_{\mathrm{NIC}}$ is used to ensure that $\mathcal{F}_{\mathrm{ZK}}^{R}$ receives the input read from $\mathcal{F}_{\mathrm{CD}}$. $\mathcal{V}$ learns neither what value is read nor the position where it is stored.

The protocol for an OR proof is more efficient when the values in $\mathrm{Tbl}_{c d}$ are known by both $\mathcal{P}$ and $\mathcal{V}$. In this case, $\mathcal{V}$ sets up the database, and then $\mathcal{P}$ performs a read operation.

When $\mathrm{Tbl}_{c d}$ needs to be hidden from $\mathcal{V}$, if the database size is $N_{\max }, \mathcal{P}$ needs to perform $N_{\max }$ write operations to write the database values into $\mathrm{Tbl}_{c d}$. Therefore, this case is similar to the worst case for computation cost described in Section VI-C. $\mathcal{P}$ needs to compute a VC witness for each of the database positions with a computation cost that grows quadratically with $N_{\max }$. Still, this cost can be amortized if the number of read operations performed subsequently is big compared to $N_{\max }$. Zero-Knowledge counting. In Appendix L, Appendix $\mathrm{M}$ and Appendix N, we describe the use of $\mathcal{F}_{\mathrm{CD}}$ to construct a protocol for "zero-knowledge counting", which roughly speaking is about counting the number of times each possible witness is used in the computation of different $\mathrm{ZK}$ proofs. $\mathrm{ZK}$ counting is a task parameterized by a relation $R$. For the possible witness values wit such that, for any instance ins, (wit, ins $) \in R$, ZK counting consists in counting how many times each witness value wit was used by $\mathcal{P}$. We define an ideal functionality $\mathcal{F}_{\mathrm{ZKC}}^{R}$ for $\mathrm{ZK}$ counting that stores one counter for each possible witness value. When $\mathcal{P}$ sends (wit, ins) $\in R$, $\mathcal{F}_{\mathrm{ZKC}}^{R}$ increments the counter for the witness value wit. At this stage, no information about the witness used is revealed to $\mathcal{V}$, but later $\mathcal{F}_{\mathrm{ZKC}}^{R}$ allows $\mathcal{P}$ to disclose to $\mathcal{V}$ the value of the counter for a given witness.

We construct $\mathrm{ZK}$ counting by using $\mathcal{F}_{\mathrm{CD}}$ as a building block. Basically, the array stored by $\mathcal{F}_{\mathrm{CD}}$ stores the counters for each of the witness values. Each position in the array is associated with one of the witness values. $\mathcal{F}_{\mathrm{ZK}}^{R_{c}}$ is used to prove in $\mathrm{ZK}$ that a counter is correctly incremented. $\mathcal{F}_{\mathrm{NIC}}$ is used to guarantee that equality between the counter read from $\mathcal{F}_{\mathrm{CD}}$ and the one input to $\mathcal{F}_{\mathrm{ZK}}^{R_{c}}$, and also to guarantee equality between the updated counter input to $\mathcal{F}_{\mathrm{ZK}}^{R_{c}}$ and the counter written into $\mathcal{F}_{\mathrm{CD}}$.

$\mathrm{ZK}$ counting is useful for the collection of aggregate statistics about $\mathcal{P}$. We describe two applications of $\mathrm{ZK}$ counting to privacy-preserving e-commerce and to privacy-preserving location-sharing services. In this applications, the witnesses represent types of items for sale and locations respectively, and the counters represent the number of times users purchased an item or checked-in at a location. Therefore, for privacy protection, $\mathcal{P}$ needs to hide both witnesses and counters from $\mathcal{V}$. $\mathcal{P}$ also needs to prove in $\mathrm{ZK}$ statements about them. For example, $\mathcal{P}$ must prove that she increments the counter for the item that she purchases.

Privacy-Preserving E-Commerce. Priced oblivious transfer (POT) is a protocol between a seller and a buyer that can be applied to e-commerce of digital goods. The seller sells messages $\left(m_{1}, \ldots, m_{N}\right)$. The prices of the messages are $\left(p_{1}\right.$, $\left.\ldots, p_{N}\right)$. At each purchase, the buyer chooses $\sigma \in[1, N]$ and obtains message $m_{\sigma}$. The seller does not learn any information about $\sigma$, but is guaranteed that the buyer does not learn any information about messages different from $m_{\sigma}$. Some constructions of POT use zero-knowledge proofs as building block [11], [29]. At each purchase, a buyer uses $\sigma$ as witness in order to prove in zero-knowledge that she purchases message $m_{\sigma}$ and pays the price $p_{\sigma}$.

POT schemes use a prepaid mechanism [2], [11], [29]. The buyer pays an initial deposit to the seller. When the buyer purchases $m_{\sigma}$, the buyer subtracts the price $p_{\sigma}$ from the deposit in such a way that the seller learns neither $p_{\sigma}$ nor the new value of the deposit.

Recently, in [30], $\mathcal{F}_{\mathrm{CD}}$ is used as building block in the construction of a POT scheme that allows the seller to obtain aggregate statistics about the purchases of a buyer. Seller and buyer execute multiple POT protocols where each of the indices $[1, N]$ is associated with a type of digital content. $\mathcal{F}_{\mathrm{CD}}$ is used to store a database of $N$ counters, where the counter at position $i \in[1, N]$ counts the number of times the buyer has purchased messages associated with index $i$. At each purchase, the corresponding counter is incremented. We remark that hiding the position of the counter that is updated is crucial to maintain the privacy properties of POT. $\mathcal{F}_{\mathrm{CD}}$ is also used to store the buyer's deposit, which is updated at each purchase.

As described in [30], $\mathcal{F}_{\mathrm{CD}}$ allows the design of the first POT protocol where the seller obtains statistics about the buyer's purchases. Aggregate statistics about multiple buyers are also possible by using a secure multiparty computation protocol on input the committed database of each of the buyers.

As explained in [30], the use of $\mathcal{F}_{\mathrm{CD}}$ introduces little overhead in comparison to previous POT protocols [11], [29] that do not gather statistics. The reason is that previous protocols already needed a database (in the form of a commitment) to store and update the deposit. With $\Pi_{\mathrm{CD}}$ the database also stores the counters $N$, but the communication cost and amortized computation cost of reading and writing the database is independent of $N$. Furthermore, this application of $\mathcal{F}_{\mathrm{CD}}$ mirrors the best case for computation cost described in Section VI-C. Namely, from the probably large number $N$ of messages sold by the seller, the average buyer is likely to purchase a small subset, so only a small subset of positions is read and written. Additionally, the counters are initialized to zero.

Privacy-Preserving Location-Sharing Services. In locationsharing services, a user checks in at a venue (e.g., a restaurant or shop) and reports her location to the service provider. The service provider sends the user's location to the user's registered friends.

In a privacy-preserving location-sharing service, a user encrypts her location and sends it to the service provider [4]. The service provider forwards the encrypted location to the user's registered friends, but does not learn the location himself. Still, the service provider requires the user to prove in $\mathrm{ZK}$ that the encrypted location is a venue registered with the service provider. There are different ways this $\mathrm{ZK}$ proof can 
be computed. For example, the service provider can issue a list of signatures where each signature signs a registered venue. The user then picks the signature that signs the venue where she checks in and proves in ZK that the encrypted message equals a message signed by the service provider.

In this setting, $\mathcal{F}_{\mathrm{CD}}$ can be used to allow the service provider to obtain aggregate information about the venues where the user checks in. Each time a user checks in at a venue, the user uses the identifier of the venue as witness in the $\mathrm{ZK}$ proof. By using $\mathcal{F}_{\mathrm{CD}}$, the protocol can be augmented to count the number of times each of the venues' identifiers is used as witness by the user, and to eventually reveal to the service provider aggregate data about the venues where the user checks in. This could be used by users, e.g., to obtain discounts at venues where they have checked-in a certain number of times. We remark that this application of $\mathcal{F}_{\mathrm{CD}}$ is possible thanks to the fact that, when a counter is incremented, $\mathcal{F}_{\mathrm{CD}}$ hides from the provider both the counter value and the witness associated to that counter.

In terms of efficiency, the communication cost and amortized computation cost of our construction for $\mathcal{F}_{\mathrm{CD}}$ are independent of $N$. Moreover, this application of $\mathcal{F}_{\mathrm{CD}}$ also mirrors the best case for computation cost, i.e., the average user is likely to check-in at a small subset of the probably large number $N$ of venues offered by the provider, and the counters are initialized at 0 .

Privacy-Preserving Billing. In privacy-preserving billing [3], a meter measures the consumption of a service by a user and outputs signed meter readings. The service provider establishes a tariff policy. To protect user privacy, the user computes the price to be paid for all the meter readings in a billing period and proves in $\mathrm{ZK}$ that the price is correct according to the signed meter readings and the tariff policy.

$\mathcal{F}_{\mathrm{CD}}$ can be used in this setting to allow the provider to get aggregate statistics about user consumption and to enable history-dependent policies. For example, the provider can offer discounts to users whose consumption does not exceed a threshold during e.g. 90 per cent of time on high-demand periods. Using $\mathcal{F}_{\mathrm{CD}}$, the user can store her consumption readings in the database at positions that represent the time of consumption, and then prove in $\mathrm{ZK}$ that she fulfills the policy without disclosing the times of consumption used.

\section{RELATED WORK}

Several cryptographic primitives allow us to represent a number of values in a much smaller cryptographic artifact and later prove knowledge of a number represented in the artifact. We summarize these primitives and compare them to our solution. In Appendix $\mathrm{O}$, we give a more detailed review of those primitives.

Commitments and ZK proofs of shuffles. Many protocols (e.g. [1], [10], [11]) use commitment schemes to maintain a database between a prover $\mathcal{P}$ and a verifier $\mathcal{V}$. However, commitments on their own are not adequate to realize $\mathcal{F}_{\mathrm{CD}}$ because they do not allow $\mathcal{P}$ to hide the positions read from or written into the database. ZK proofs of shuffles [14] can be used to shuffle the commitments in order to hide the positions read or written. A construction using commitments along with proofs of shuffles could realize $\mathcal{F}_{\mathrm{CD}}$, but less efficiently than a construction based on VCs.

Accumulators. A cryptographic accumulator [31] allows one to represent a set $X$ succinctly as a single accumulator value $A$. It also provides a method to prove succinctly that an element $x$ belongs to $X$ to any party that holds $A$. This method consists in computing a witness $W$ whose size is independent of the size $|X|$ of the set. Soundness (or collision-freeness) guarantees that it is infeasible to prove that $x \in X$ if $x \notin X$. The main difference between VCs and accumulators is that, while accumulators allow for committing to a set, VCs allow for committing to a vector of messages, where each message is committed at a specific position. This allows the construction of an updatable committed database where it is also possible to prove statements about the position where a message is written or read.

Vector Commitments. In [7], a definition of non-hiding VCs with updates is given. To obtain hiding VCs, it is suggested to compose a non-hiding VC scheme with a standard commitment scheme. Two constructions of non-hiding VCs are given based on the CDH and RSA assumptions. In [6], a construction of mercurial VCs based on the Diffie-Hellman Exponent (DHE) assumption is proposed. This construction leads to constructions of non-hiding and hiding VCs based on DHE, which were used in, e.g., [32]-[34].

Our committed database uses as building block any hiding VC scheme with updates, along with ZK proofs of knowledge that a vector component is being read or written. To instantiate our committed database, we use a construction of hiding VCs with updates based on the DHE assumption along with the corresponding $\mathrm{ZK}$ proofs for reading and writing. In this construction, the size of the public parameters is linear in the maximum vector length. In comparison, in a hiding VC construction from $\mathrm{CDH}$, the size of the public parameters would be quadratic (the advantage would be to use a more standard assumption).

Recently, in [35], subvector commitments (SVC) are proposed. In SVC, a commitment can be opened to a set of positions such that the size of the witness does not depend on the size of the set. A construction for SVC secure under the cube Diffie-Hellman assumption is given, in which the public parameter size grows quadratically with the vector length. $\mathcal{F}_{\mathrm{CD}}$ and our applications for it in Section VIII only require to open one vector component at a time. SVC can be used to construct a variant of $\mathcal{F}_{\mathrm{CD}}$ where several positions are read or written simultaneously. Nevertheless, we note that, despite that SVC provides witnesses of size independent of the number of positions open, the entire witness of read or write proofs would still grow with the number of positions, and thus the efficiency of those proofs would not be independent of the set size. In [35], [36], constructions for SVC based on groups of hidden order are proposed that are better suited for bit vectors. Polynomial and functional commitments. Polynomial commitments allow a committer to commit to a polynomial and 
open the commitment to an evaluation of the polynomial. They can be used as VCs by committing to a polynomial that interpolates the vector to be committed. In [12], a construction of polynomial commitments from the SDH assumption is proposed, which has the disadvantage that efficient updates cannot be computed without knowledge of the trapdoor. A further generalization of VCs and polynomial commitments are functional commitments [13], [35].

Zero-Knowledge Data Structures. Zero-Knowledge Sets (ZKS) [15] allow a prover $\mathcal{P}$ to commit to a set $X$ and to subsequently prove to a verifier $\mathcal{V}$ (non-)membership of an element $x$ in $X$. Zero-Knowledge Databases (ZKDB) are similar to ZKS but each element $x \in X$ is associated with a value $v$, in such a way that a proof that $x \in X$ reveals $v$ to $\mathcal{V}$. Both ZKS and ZKDB are two-party protocols between $\mathcal{P}$ and $\mathcal{V}$. Security for $\mathcal{V}$ requires that an adversarial $\mathcal{P}$ is not able to prove $x \in X$ if $x \notin X$ (and vice versa), while zero-knowledge requires that proofs of (non-)membership reveal nothing else beyond (non-)membership, not even the size of the set. In [19], a construction for zero-knowledge lists (ZKL) is proposed, where a list is defined as an ordered set. Updatable ZKDB were first proposed in [18]. In [7], a construction for updatable ZKDB based on updatable VCs and trapdoor mercurial commitments is proposed.

There are several differences between our committed database and previous work. First, our committed database is updatable, which was only considered in [7], [18]. Second, our committed database is oblivious. $\mathcal{P}$ proves in $\mathrm{ZK}$ that a pair of commitments commit to a position and value that are stored in the database. In contrast, in previous constructions, $\mathcal{P}$ reveals a position and a value along with a proof that the position and the value are stored in the database. The obliviousness property allows our committed database to be used as building block in applications that protect the privacy of the $\mathcal{P}$, because $\mathcal{P}$ could choose to open the commitments, but could also prove statements in $\mathrm{ZK}$ about the committed position and value without revealing them.

From a definitional point of view, security definitions given in previous works are not in the UC model and a mechanism to integrate modularly ZKS or ZKDB as building blocks of other protocols is not given. Our functionality for a committed database allows the security analysis of $\mathrm{ZK}$ data structures in a composable framework, which will facilitate the modular design and analysis of protocols that use them as a building block.

Another key difference is that we do not require the size of the database to be hidden. Thanks to that, our construction for a committed database is more efficient than existing constructions for ZKS or ZKDB. This relaxation of the ZK property is not relevant for our applications for a committed database. In this respect, our construction for a committed database is similar to the constructions for "nearly" ZKS and ZKDB given in [12]. However, this "nearly" ZKS and ZKDB constructions based on the SDH assumption are not updatable and, moreover, extending them with efficient updates is not possible without knowledge of the trapdoor. In fact, as pointed out in [37], when hiding the size of the database is required, for any construction that uses a non-interactive commitment phase (as is the case in the ZKS and ZKDB constructions cited above), black-box extraction of the database by the simulator in the security proof is not possible. In [37], a secure committed database where the database size is hidden is defined in the UC model, and a construction that uses an interactive commitment phase is proposed. As a consequence of needing to hide the database size, the construction in [37] is also less efficient than ours. Also, their ideal functionality does not facilitate modular design, and it outputs position-value pairs instead of commitments to a position and to a value, which hinders its use as building block in protocols that need to protect the privacy of the prover.

In [38], a functionality for a database such that both prover and verifier know the database contents is proposed. The verifier can write information into the database, while the prover performs a read operation similar to the one of $\mathcal{F}_{\mathrm{CD}}$. Non-hiding VCs are used to construct the functionality. In [39], a variant of the functionality in [38] that interacts with multiple provers and provides unlinkable read operations is defined and constructed by using SVCs.

ZK proofs for large datasets. In most $\mathrm{ZK}$ proofs, the computation and communication cost grow linearly with the size of the witness, which is inadequate for proofs about datasets $M$ of large size $|M|$. However, there are techniques that attain costs sublinear in $|M|$. Probabilistically checkable proofs [40] achieve verification cost sublinear in $|M|$, but the cost for the prover is linear in $|M|$. In succinct non-interactive arguments of knowledge [41], verification cost is independent of $|M|$, but the cost for the prover is still linear in $|M|$.

$\mathrm{ZK}$ proofs for relations described as ORAM programs [8], [9] involve an initialization phase in which the prover commits to $M$. In [8], the cost for the prover is linear in $|M|$, whereas the cost for the verifier is independent of $|M|$. After the initialization phase, many proofs can be computed about $M$ whose cost is sublinear (proportional to the runtime of the ORAM program) both for the prover and for the verifier. The protocol in [8] uses a non-programmable random oracle, which is key for achieving constant cost for the verifier in the initialization phase. Any protocol in the standard model would involve communication cost linear in $M$ to allow knowledge extraction.

To compare our protocol with [8], we consider the setting of an OR proof when the database must be hidden from $\mathcal{V}$, i.e., $\mathcal{P}$ writes $M$ into $\mathrm{Tbl}_{c d}$, and after that reads values in $M$ from $\mathrm{Tbl}_{c d}$. Assuming the worst case of all non-zero values in $M$, the cost of writing $M$ into $\mathrm{Tbl}_{c d}$ is quadratic in $|M|$ for $\mathrm{Tbl}_{c d}$ and linear in $|M|$ for $\mathcal{V}$. However, after that the cost of each read operation is independent of $|M|$ for both $\mathcal{P}$ and $\mathcal{V}$. Our protocol provides thus better asymptotic amortized cost than the state of the art protocol in [8] when the number of read operations is big compared to $|M|$. (In the initialization phase, the cost for the verifier is linear in $|M|$ which is unavoidable when aiming for security in the standard CRS-hybrid model.) We note that [8] does not provide a concrete instantiation or efficiency analysis of their protocol, so we do not compare it 
with the instantiation of our protocol in Section VI.

\section{COnClusion And Future Work}

We have made a couple of design choices in $\mathcal{F}_{\mathrm{CD}}$. For instance, all parties are authenticated and the functionality implies interaction between $\mathcal{P}$ and $\mathcal{V}$. Our functionality could be extended, depending on the requirements of potential applications. For instance, a non-interactive functionality would allow for applications with multiple verifiers. Another extension of $\mathcal{F}_{\mathrm{CD}}$ could provide pseudonymity or anonymity, which would be suitable for applications such as attribute-based credentials [10]. $\mathcal{F}_{\mathrm{CD}}$ is suitable for protocols in which a onedimensional array is adequate to implement the database. $\mathcal{F}_{\mathrm{CD}}$ could be extended to more complex updatable data structures, such as multi-dimensional arrays, lists, trees, and graphs. Some of these would require operations beyond read and write.

Acknowledgements. Alfredo Rial is supported by the Luxembourg National Research Fund (FNR) CORE project "Stateful Zero-Knowledge" (Project code: C17/11650748).

\section{REFERENCES}

[1] R. Canetti, Y. Lindell, R. Ostrovsky, and A. Sahai, "Universally composable two-party and multi-party secure computation," in STOC 2002, pp. 494-503.

[2] W. Aiello, Y. Ishai, and O. Reingold, "Priced oblivious transfer: How to sell digital goods," in EUROCRYPT 2001, pp. 119-135.

[3] A. Rial and G. Danezis, "Privacy-preserving smart metering," in WPES 2011, pp. 49-60.

[4] M. Herrmann, A. Rial, C. Díaz, and B. Preneel, "Practical privacypreserving location-sharing based services with aggregate statistics," in WiSec'14, pp. 87-98.

[5] J. Camenisch, M. Dubovitskaya, and A. Rial, "UC commitments for modular protocol design and applications to revocation and attribute tokens," in CRYPTO 2016, pp. 208-239.

[6] B. Libert and M. Yung, "Concise mercurial vector commitments and independent zero-knowledge sets with short proofs," in TCC 2010, pp 499-517.

[7] D. Catalano and D. Fiore, "Vector commitments and their applications," in PKC 2013, pp. 55-72.

[8] P. Mohassel, M. Rosulek, and A. Scafuro, "Sublinear zero-knowledge arguments for RAM programs," in EUROCRYPT 2017, pp. 501-531.

[9] Z. Hu, P. Mohassel, and M. Rosulek, "Efficient zero-knowledge proofs of non-algebraic statements with sublinear amortized cost," in CRYPTO 2015, pp. 150-169.

[10] J. Camenisch and A. Lysyanskaya, "An efficient system for nontransferable anonymous credentials with optional anonymity revocation," in EUROCRYPT 2001, pp. 93-118.

[11] A. Rial, M. Kohlweiss, and B. Preneel, "Universally composable adaptive priced oblivious transfer," in Pairing 2009, pp. 231-247.

[12] A. Kate, G. M. Zaverucha, and I. Goldberg, "Constant-size commitment to polynomials and their applications," in ASIACRYPT 2010, pp. 177-194

[13] B. Libert, S. C. Ramanna, and M. Yung, "Functional commitment schemes: From polynomial commitments to pairing-based accumulators from simple assumptions," in ICALP 2016, pp. 30:1-30:14.

[14] M. Chase, M. Kohlweiss, A. Lysyanskaya, and S. Meiklejohn, "Malleable proof systems and applications," in EUROCRYPT 2012, pp. 281-300.

[15] S. Micali, M. O. Rabin, and J. Kilian, "Zero-knowledge sets," in FOCS 2003, pp. 80-91.

[16] M. Chase, A. Healy, A. Lysyanskaya, T. Malkin, and L. Reyzin, "Mercurial commitments with applications to zero-knowledge sets," $J$. Cryptology, vol. 26, no. 2, pp. 251-279, 2013.

[17] D. Catalano, D. Fiore, and M. Messina, "Zero-knowledge sets with short proofs," in EUROCRYPT 2008, pp. 433-450.

[18] M. Liskov, "Updatable zero-knowledge databases," in ASIACRYPT 2005, 2005, pp. 174-198.

[19] E. Ghosh, O. Ohrimenko, and R. Tamassia, "Zero-knowledge authenticated order queries and order statistics on a list," in ACNS 2015, pp. 149-171.
[20] E. Ghosh, M. T. Goodrich, O. Ohrimenko, and R. Tamassia, "Verifiable zero-knowledge order queries and updates for fully dynamic lists and trees," in SCN 2016, pp. 216-236.

[21] R. Ostrovsky, C. Rackoff, and A. D. Smith, "Efficient consistency proofs for generalized queries on a committed database," in ICALP 2004, pp. 1041-1053.

[22] S. Goldberg, M. Naor, D. Papadopoulos, L. Reyzin, S. Vasant, and A. Ziv "NSEC5: provably preventing DNSSEC zone enumeration," in NDSS 2015, 2015.

[23] R. Canetti, "Universally composable security: A new paradigm for cryptographic protocols," Cryptology ePrint Archive, Report 2000/067, 2000, https://eprint.iacr.org/2000/067.

[24] _ - "Universally composable security: A new paradigm for cryptographic protocols," in FOCS 2001, pp. 136-145.

[25] J. Camenisch, S. Krenn, and V. Shoup, "A framework for practical universally composable zero-knowledge protocols," in ASIACRYPT 2011, pp. 449-467.

[26] S. Goldwasser, S. Micali, and R. L. Rivest, "A digital signature scheme secure against adaptive chosen-message attacks," SIAM J. Comput. vol. 17 , no. 2, pp. 281-308, 1988 .

[27] M. Abe, J. Groth, K. Haralambiev, and M. Ohkubo, "Optimal structurepreserving signatures in asymmetric bilinear groups," in CRYPTO 2011, pp. 649-666.

[28] T. P. Pedersen, "Non-interactive and information-theoretic secure verifiable secret sharing," in CRYPTO '91, pp. 129-140.

[29] J. Camenisch, M. Dubovitskaya, and G. Neven, "Unlinkable priced oblivious transfer with rechargeable wallets," in FC 2010, pp. 66-81.

[30] A. Damodaran, M. Dubovitskaya, and A. Rial, "UC priced oblivious transfer with purchase statistics and dynamic pricing," in INDOCRYPT 2019, pp. 273-296.

[31] J. C. Benaloh and M. de Mare, "One-way accumulators: A decentralized alternative to digital sinatures (extended abstract),' in EUROCRYPT '93, pp. 274-285.

[32] B. Libert, T. Peters, and M. Yung, "Group signatures with almost-for-free revocation," in CRYPTO 2012, pp. 571-589.

[33] M. Izabachène, B. Libert, and D. Vergnaud, "Block-wise p-signatures and non-interactive anonymous credentials with efficient attributes," in Cryptography and Coding - 13th IMA International Conference, IMACC 2011, pp. 431-450.

[34] M. Kohlweiss and A. Rial, "Optimally private access control," in WPES 2013, pp. 37-48.

[35] R. W. F. Lai and G. Malavolta, "Subvector commitments with application to succinct arguments," in CRYPTO 2019, pp. 530-560.

[36] D. Boneh, B. Bünz, and B. Fisch, "Batching techniques for accumulators with applications to iops and stateless blockchains," in CRYPTO 2019, pp. 561-586.

[37] M. Chase and I. Visconti, "Secure database commitments and universal arguments of quasi knowledge," in CRYPTO 2012, pp. 236-254.

[38] A. Damodaran and A. Rial, "UC updatable databases and applications," in AFRICACRYPT 2020, pp. 66-87.

[39] _ , "Unlinkable updatable databases and oblivious transfer with access control," in ACISP 2020, pp. 584-604.

[40] J. Kilian, "A note on efficient zero-knowledge proofs and arguments (extended abstract)," in STOC 1992, pp. 723-732.

[41] R. Gennaro, C. Gentry, B. Parno, and M. Raykova, "Quadratic span programs and succinct nizks without pcps," in EUROCRYPT 2013, pp. 626-645.

[42] D. Derler, C. Hanser, and D. Slamanig, "Revisiting cryptographic accumulators, additional properties and relations to other primitives,' in Topics in Cryptology - CT-RSA 2015, The Cryptographer's Track at the RSA Conference 2015, San Francisco, CA, USA, April 20-24, 2015. Proceedings, 2015, pp. 127-144.

[43] K. Nyberg, "Fast accumulated hashing," in Fast Software Encryption Third International Workshop, Cambridge, UK, February 21-23, 1996, Proceedings, 1996, pp. 83-87.

[44] T. Sander, "Efficient accumulators without trapdoor extended abstracts," in ICICS'99, pp. 252-262.

[45] P. Camacho, A. Hevia, M. A. Kiwi, and R. Opazo, "Strong accumulators from collision-resistant hashing," in ISC 2008, pp. 471-486.

[46] H. Lipmaa, "Secure accumulators from euclidean rings without trusted setup," in ACNS 2012, pp. 224-240.

[47] J. Camenisch and A. Lysyanskaya, "Dynamic accumulators and application to efficient revocation of anonymous credentials," in CRYPTO 2002, pp. 61-76. 
[48] M. H. Au, P. P. Tsang, W. Susilo, and Y. Mu, "Dynamic universal accumulators for DDH groups and their application to attribute-based anonymous credential systems," in CT-RSA 2009, pp. 295-308.

[49] L. Nguyen, "Accumulators from bilinear pairings and applications," in CT-RSA 2005, pp. 275-292.

[50] J. Camenisch, M. Kohlweiss, and C. Soriente, "An accumulator based on bilinear maps and efficient revocation for anonymous credentials," in PKC 2009, pp. 481-500.

[51] E. Ghosh, O. Ohrimenko, D. Papadopoulos, R. Tamassia, and N. Triandopoulos, "Zero-knowledge accumulators and set algebra," in ASIACRYPT 2016, pp. 67-100.

[52] J. Li, N. Li, and R. Xue, "Universal accumulators with efficient nonmembership proofs," in ACNS 2007, pp. 253-269.

[53] D. Catalano, Y. Dodis, and I. Visconti, "Mercurial commitments: Minimal assumptions and efficient constructions," in TCC 2006, pp. 120-144.

[54] R. Tamassia, "Authenticated data structures," in Algorithms - ESA 2003, 11th Annual European Symposium, Budapest, Hungary, September 16-19, 2003, Proceedings, 2003, pp. 2-5.

[55] H. C. Pöhls and K. Samelin, "On updatable redactable signatures," in ACNS 2014, pp. 457-475.

\section{APPENDIX A}

\section{UNIVERSALLy COMPOSABLE SECURITY}

The universal composability framework [24] is a framework for defining and analyzing the security of cryptographic protocols so that security is retained under arbitrary composition with other protocols. The security of a protocol is defined by means of an ideal protocol that carries out the desired task. In the ideal protocol, all parties send their inputs to an ideal functionality $\mathcal{F}$ for the task. The ideal functionality locally computes the outputs of the parties and provides each party with its prescribed output.

The security of a protocol $\varphi$ is analyzed by comparing the view of an environment $\mathcal{Z}$ in a real execution of $\varphi$ against that of $\mathcal{Z}$ in the ideal protocol defined in $\mathcal{F}_{\varphi}$. The environment $\mathcal{Z}$ chooses the inputs of the parties and collects their outputs. In the real world, $\mathcal{Z}$ can communicate freely with an adversary $\mathcal{A}$ who controls both the network and any corrupt parties. In the ideal world, $\mathcal{Z}$ interacts with dummy parties, who simply relay inputs and outputs between $\mathcal{Z}$ and $\mathcal{F}_{\varphi}$, and a simulator $\mathcal{S}$. We say that a protocol $\varphi$ securely realizes $\mathcal{F}_{\varphi}$ if $\mathcal{Z}$ cannot distinguish the real world from the ideal world, i.e., $\mathcal{Z}$ cannot distinguish whether it is interacting with $\mathcal{A}$ and parties running protocol $\varphi$ or with $\mathcal{S}$ and dummy parties relaying to $\mathcal{F}_{\varphi}$.

A protocol $\varphi^{\mathcal{G}}$ securely realizes $\mathcal{F}$ in the $\mathcal{G}$-hybrid model when $\varphi$ is allowed to invoke the ideal functionality $\mathcal{G}$. Therefore, for any protocol $\psi$ that securely realizes the functionality $\mathcal{G}$, the composed protocol $\varphi^{\psi}$, which is obtained by replacing each invocation of an instance of $\mathcal{G}$ with an invocation of an instance of $\psi$, securely realizes $\mathcal{F}$.

In the ideal functionalities described in this paper, we consider static corruptions. When describing ideal functionalities, we use the following conventions as in [5].

Interface Naming Convention. An ideal functionality can be invoked by using one or more interfaces. The name of a message in an interface consists of three fields separated by dots, e.g., cd.read.ini in the committed database functionality described in Section III. The first field indicates the name of the functionality and is the same in all interfaces of the functionality. This field is useful for distinguishing between invocations of different functionalities in a hybrid protocol that uses two or more different functionalities. The second field indicates the kind of action performed by the functionality and is the same in all messages that the functionality exchanges within the same interface. The third field distinguishes between the messages that belong to the same interface, and can take six different values. A message $* . *$.ini is the incoming message received by the functionality, i.e., the message through which the interface is invoked. A message $* . *$.end is the outgoing message sent by the functionality, i.e., the message that ends the execution of the interface. The message $* . * . s i m$ is used by the functionality to send a message to the simulator, and the message $*$.* .rep is used to receive a message from the simulator. The message $* *$.req is used by the functionality to send a message to the simulator to request the description of algorithms from the simulator, and the message *.* alg is used by the simulator to send the description of those algorithms to the functionality.

Network vs local communication. The identity of an interactive Turing machine (ITM) instance (ITI) consists of a party identifier $p i d$ and a session identifier sid. A set of parties in an execution of a system of ITMs is a protocol instance if they have the same session identifier sid. ITIs can pass direct inputs to and outputs from "local" ITIs that have the same pid. An ideal functionality $\mathcal{F}$ has pid $=\perp$ and is considered local to all parties. An instance of $\mathcal{F}$ with the session identifier sid only accepts inputs from and passes outputs to machines with the same session identifier sid. Some functionalities require the session identifier to have some structure. Those functionalities check whether the session identifier possesses the required structure in the first message that invokes the functionality. For the subsequent messages, the functionality implicitly checks that the session identifier equals the session identifier used in the first message. Communication between ITIs with different party identifiers must take place over the network. The network is controlled by the adversary, meaning that he can arbitrarily delay, modify, drop, or insert messages.

Query identifiers. Some interfaces in a functionality can be invoked more than once. When the functionality sends a message *.* sim to the simulator in such an interface, a query identifier qid is included in the message. The query identifier must also be included in the response *.* .rep sent by the simulator. The query identifier is used to identify the message $* . *$.sim to which the simulator replies with a message $* . *$.rep. We note that, typically, the simulator in the security proof may not be able to provide an immediate answer to the functionality after receiving a message $* . *$.sim. The reason is that the simulator typically needs to interact with the copy of the real adversary it runs in order to produce the message *.*.rep, but the real adversary may not provide the desired answer or may provide a delayed answer. In such cases, when the functionality sends more than one 
message *.*.sim to the simulator, the simulator may provide delayed replies, and the order of those replies may not follow the order of the messages received.

Aborts. When an ideal functionality $\mathcal{F}$ aborts after being activated with a message sent by a party, we mean that $\mathcal{F}$ halts the execution of its program and sends a special abortion message to the party that invoked the functionality. When an ideal functionality $\mathcal{F}$ aborts after being activated with a message sent by the simulator, we mean that $\mathcal{F}$ halts the execution of its program and sends a special abortion message to the party that receives the outgoing message from $\mathcal{F}$ after $\mathcal{F}$ is activated by the simulator.

\section{APPENDIX B \\ IDEAL FUNCTIONALITY $\mathcal{F}_{\text {CRS }}^{\text {CRS.Setup }}$}

Our protocol uses the functionality $\mathcal{F}_{\text {CRS }}^{\text {CRS.Setup }}$ for common

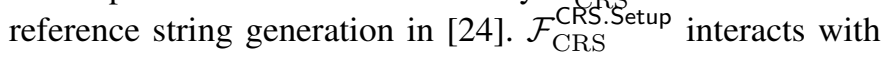
any parties $\mathcal{P}$ that obtain the common reference string, and consists of one interface crs.get. A party $\mathcal{P}$ uses the crs.get interface to request and receive the common reference string $\mathrm{crs}$ from $\mathcal{F}_{\mathrm{CRS}}^{\text {CRS.Setup }}$. In the first invocation, $\mathcal{F}_{\mathrm{CRS}}^{\text {CRS.Setup generates }}$ crs by running algorithm CRS.Setup. The simulator $\mathcal{S}$ also

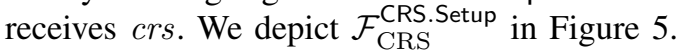

\section{APPENDIX C \\ IDEAL FUNCTIONALITY $\mathcal{F}_{\text {AUT }}$}

Our protocol uses the functionality $\mathcal{F}_{\text {AUT }}$ for an authenticated channel in [24]. $\mathcal{F}_{\text {AUT }}$ interacts with a sender $\mathcal{T}$ and a receiver $\mathcal{R}$, and consists of one interface aut.send. $\mathcal{T}$ uses the aut.send interface to send a message $m$ to $\mathcal{F}_{\text {AUT }}$. $\mathcal{F}_{\text {AUT }}$ leaks $m$ to the simulator $\mathcal{S}$ and, after receiving a response from $\mathcal{S}, \mathcal{F}_{\text {AUT }}$ sends $m$ to $\mathcal{R}$. $\mathcal{S}$ cannot modify $m$. The session identifier sid contains the identities of $\mathcal{T}$ and $\mathcal{R}$. We depict $\mathcal{F}_{\text {AUT }}$ in Figure 6.

\section{APPENDIX D \\ IDEAL Functionality $\mathcal{F}_{\mathrm{ZK}}^{R}$ FOR ZERO-KNOWLEDGE}

Let $R$ be a polynomial time computable binary relation. For tuples (wit, ins $) \in R$ we call wit the witness and ins the instance. Our protocol uses the ideal functionality $\mathcal{F}_{\mathrm{ZK}}^{R}$ for zero-knowledge in [24]. $\mathcal{F}_{\mathrm{ZK}}^{R}$ is parameterized by a description of a relation $R$, runs with a prover $\mathcal{P}$ and a verifier $\mathcal{V}$, and consists of one interface zk. prove. $\mathcal{P}$ uses zk. prove to send a witness wit and an instance ins to $\mathcal{F}_{\mathrm{ZK}}^{R} . \mathcal{F}_{\mathrm{ZK}}^{R}$ checks whether (wit, ins) $\in R$, and, in that case, sends the instance ins to $\mathcal{V}$. The simulator $\mathcal{S}$ learns ins but not wit. We depict $\mathcal{F}_{\mathrm{ZK}}^{R}$ in Figure 7.

\section{APPENDIX E}

\section{IDEAL FUNCTIONALITY $\mathcal{F}_{\text {NIC FOR NON-INTERACTIVE }}$} COMMITMENTS

Our protocol uses the functionality $\mathcal{F}_{\text {NIC }}$ for non-interactive commitments in [5]. $\mathcal{F}_{\mathrm{NIC}}$ interacts with parties $\mathcal{P}_{i}$ and consists of the following interfaces:

1) Any party $\mathcal{P}_{i}$ uses the com.setup interface to set up the functionality.
2) Any party $\mathcal{P}_{i}$ uses the com.commit interface to send a message $m$ and obtain a commitment com and an opening open. A commitment $\mathrm{com}=\left(\mathrm{com}^{\prime}\right.$, parcom, COM.Verify), where $\mathrm{com}^{\prime}$ is the commitment, parcom are the public parameters, and COM.Verify is the verification algorithm.

3) Any party $\mathcal{P}_{i}$ uses the com.validate interface to send a commitment com in order to check that com contains the correct public parameters and verification algorithm.

4) Any party $\mathcal{P}_{i}$ uses the com.verify interface to send (com, $m$,open) in order to verify that com is a commitment to the message $m$ with the opening open.

$\mathcal{F}_{\text {NIC }}$ can be realized by a perfectly hiding commitment scheme, such as Pedersen commitments [5]. In [5], a method is described to use $\mathcal{F}_{\text {NIC }}$ in order to ensure that a party sends the same input $m$ to several ideal functionalities. For this purpose, the party first uses com.commit to get a commitment com to $m$ with opening open. Then the party sends (com, m,open) as input to each of the functionalities, and each functionality runs COM.Verify to verify the commitment. Finally, other parties in the protocol receive the commitment com from each of the functionalities and use the com.validate interface to validate $\mathrm{com}$. Then, if com received from all the functionalities is the same, the binding property provided by $\mathcal{F}_{\mathrm{NIC}}$ ensures that all the functionalities received the same input $m$. When using $\mathcal{F}_{\text {NIC }}$, it is needed to work in the $\mathcal{F}_{\text {NIC }} \| \mathcal{S}_{\text {NIC }}$-hybrid model, where $\mathcal{S}_{\mathrm{NIC}}$ is any simulator for a construction that realizes $\mathcal{F}_{\mathrm{NIC}}$. We depict $\mathcal{F}_{\mathrm{NIC}}$ in Figure 8 .

\section{APPENDIX F IDEAL FUNCTIONALITY $\mathcal{F}_{\text {SMT }}$}

Our protocol uses the functionality $\mathcal{F}_{\mathrm{SMT}}$ for a secure channel in [24]. $\mathcal{F}_{\mathrm{SMT}}$ interacts with a sender $\mathcal{T}$ and a receiver $\mathcal{R}$, and consists of one interface smt.send. $\mathcal{T}$ uses the smt.send interface to send a message $m$ to $\mathcal{F}_{\mathrm{SMT}}$. $\mathcal{F}_{\mathrm{SMT}}$ leaks $l(m)$, where $l$ leaks the message length, to the simulator $\mathcal{S}$. After receiving a response from $\mathcal{S}, \mathcal{F}_{\mathrm{SMT}}$ sends $m$ to $\mathcal{R}$. $\mathcal{S}$ cannot modify $m$. The session identifier sid contains the identities of $\mathcal{T}$ and $\mathcal{R}$. We depict $\mathcal{F}_{\text {SMT }}$ in Figure 9 .

\section{APPENDIX G}

\section{SeCurity Definitions for Vector Commitments}

Definition G.1: A vector commitment scheme must be correct, hiding, and binding.

We define these properties in Figure 10.

\section{APPENDIX H}

\section{Construction $\Pi_{\mathrm{CD}}$ FOR A Committed Database}

Our construction $\Pi_{\mathrm{CD}}$ uses a vector commitment (VC) scheme. A vector commitment $v c$ is used to store the table $\mathrm{Tbl}_{c d}$. A position in the vector commitment acts as a position in $\mathrm{Tbl}_{c d}$, and the value committed to in that position acts as the value stored in $\mathrm{Tbl}_{c d}$ in that position. We depict $\Pi_{\mathrm{CD}}$ in Figure 11 and Figure 12.

In the setup interface, $\mathcal{P}$ and $\mathcal{V}$ obtain the VC parameters par from the functionality $\mathcal{F}_{\mathrm{CRS}}^{\mathrm{CRS} \text { Setup }}$ for common reference string, 


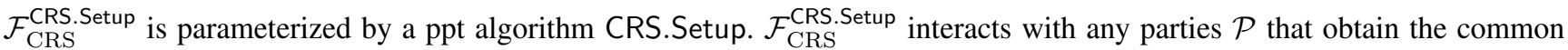
reference string:

1) On input (crs.get.ini, sid) from any party $\mathcal{P}$ :

- If (sid, crs) is not stored, run crs $\leftarrow$ CRS.Setup and store (sid, crs).

- Create a fresh qid and store $(q i d, \mathcal{P})$.

- Send (crs.get.sim, sid, qid, crs) to $\mathcal{S}$.

S. On input (crs.get.rep, sid, qid) from the simulator $\mathcal{S}$ :

- Abort if $(q i d, \mathcal{P})$ is not stored.

- Delete the record (qid, $\mathcal{P})$.

- Send (crs.get.end, sid, crs) to $\mathcal{P}$.

Fig. 5. Functionality $\mathcal{F}_{\text {CRS }}^{\text {CRS.Setup }}$

$\mathcal{F}_{\text {AUT }}$ is parameterized by a message space $\mathcal{M}$.

1) On input (aut.send.ini, sid, $m$ ) from a party $\mathcal{T}$ :

- Abort if $\operatorname{sid} \neq\left(\mathcal{T}, \mathcal{R}\right.$, sid $\left.^{\prime}\right)$ or if $m \notin \mathcal{M}$.

- Create a fresh qid and store $(q i d, \mathcal{R}, m)$.

- Send (aut.send.sim, sid, qid, $m$ ) to $\mathcal{S}$.

S. On input (aut.send.rep, sid, qid) from $\mathcal{S}$ :

- Abort if $(q i d, \mathcal{R}, m)$ is not stored.

- Delete the record $(q i d, \mathcal{R}, m)$.

- Send (aut.send.end, sid, $m$ ) to $\mathcal{R}$.

Fig. 6. Functionality $\mathcal{F}_{\text {AUT }}$

$\mathcal{F}_{\mathrm{ZK}}^{R}$ is parameterized by a description of a relation $R . \mathcal{F}_{\mathrm{ZK}}^{R}$ interacts with a prover $\mathcal{P}$ and a verifier $\mathcal{V}$.

1) On input (zk.prove.ini, sid, wit, ins) from $\mathcal{P}$ :

- Abort if $\operatorname{sid} \neq\left(\mathcal{P}, \mathcal{V}\right.$, sid $\left.^{\prime}\right)$ or if (wit, ins $) \notin R$.

- Create a fresh qid and store (qid,ins).

- Send (zk.prove.sim, sid, qid, ins) to $\mathcal{S}$.

S. On input (zk.prove.rep, sid, qid) from $\mathcal{S}$ :

- Abort if (qid,ins) is not stored.

- Parse sid as $\left(\mathcal{P}, \mathcal{V}\right.$, sid $\left.^{\prime}\right)$.

- Delete the record (qid, ins).

- Send (zk.prove.end, sid, ins) to $\mathcal{V}$.

Fig. 7. Functionality $\mathcal{F}_{\mathrm{ZK}}^{R}$ 
COM.TrapCom, COM.TrapOpen and COM.Verify are ppt algorithms.

1) On input (com.setup.ini, sid) from a party $\mathcal{P}_{i}$ :

- If (sid, parcom, COM.TrapCom, COM.TrapOpen, COM.Verify, tdcom) is already stored, include $\mathcal{P}_{i}$ in the set $\mathbb{P}$, and send (com.setup.end, sid, $O K$ ) as a public delayed output to $\mathcal{P}_{i}$.

- Otherwise proceed to generate a random qid, store $\left(q i d, \mathcal{P}_{i}\right)$ and send the message (com.setup.req, sid, qid) to $\mathcal{S}$.

S. On input (com.setup.alg, sid, qid, $m$ ) from $\mathcal{S}$ :

- Abort if no pair $\left(q i d, \mathcal{P}_{i}\right)$ for some $\mathcal{P}_{i}$ is stored.

- Delete record $\left(q i d, \mathcal{P}_{i}\right)$.

- If (sid, parcom, COM.TrapCom, COM.TrapOpen, COM.Verify, tdcom) is already stored, include $\mathcal{P}_{i}$ in the set $\mathbb{P}$ and send (com.setup.end, sid, $O K$ ) to $\mathcal{P}_{i}$.

- Otherwise proceed as follows.

- $m$ is (parcom, COM.TrapCom, COM.TrapOpen, COM.Verify, tdcom).

- Initialize both an empty table $\mathrm{Tbl}_{\mathrm{com}}$ and an empty set $\mathbb{P}$, and store (sid,parcom,COM.TrapCom, COM.TrapOpen, COM.Verify, $t d c o m)$.

- Include $\mathcal{P}_{i}$ in the set $\mathbb{P}$ and send (com.setup.end, sid, OK) to $\mathcal{P}_{i}$.

2) On input (com.validate.ini, sid, com) from any party $\mathcal{P}_{i}$ :

- Abort if $\mathcal{P}_{i} \notin \mathbb{P}$.

- Parse com as $\left(\mathrm{com}^{\prime}\right.$, parcom $^{\prime}$, COM.Verify').

- Set $v \leftarrow 1$ if parcom $^{\prime}=$ parcom and COM.Verify ${ }^{\prime}=$ COM.Verify. Otherwise, set $v \leftarrow 0$.

- Send (com.validate.end, sid, $v$ ) to $\mathcal{P}_{i}$.

3) On input (com.commit.ini, sid, $m$ ) from any party $\mathcal{P}_{i}$ :

- Abort if $\mathcal{P}_{i} \notin \mathbb{P}$ or if $m \notin \mathcal{M}$, where $\mathcal{M}$ is defined in parcom.

- Compute $($ com, info $) \leftarrow$ COM.TrapCom (sid, parcom, tdcom).

- Abort if there is an entry $\left[\right.$ com, $m^{\prime}$, open $\left.^{\prime}, 1\right]$ in $\mathrm{Tbl}_{\text {com }}$ such that $m \neq m^{\prime}$.

- Run open $\leftarrow$ COM.TrapOpen $($ sid, $m$, info $)$.

- Abort if $1 \neq$ COM.Verify(sid, parcom, com, m, open).

- Append [com, m, open, 1] to Tbl com $_{\text {. }}$.

- Set com $\leftarrow$ (com, parcom, COM.Verify).

- Send (com.commit.end, sid, com, open) to $\mathcal{P}_{i}$.

4) On input (com.verify.ini, sid, com, $m$, open) from any party $\mathcal{P}_{i}$ :

- Abort if $\mathcal{P}_{i} \notin \mathbb{P}$ or if $m \notin \mathcal{M}$ or if open $\notin \mathcal{R}$, where $\mathcal{M}$ and $\mathcal{R}$ are defined in parcom.

- Parse com as the tuple $\left(\right.$ com $^{\prime}$, parcom ${ }^{\prime}$, COM.Verify $)$. Abort if the parameters parcom ${ }^{\prime} \neq$ parcom or COM.Verify $\neq$ COM.Verify.

- If there is an entry $\left[\mathrm{com}^{\prime}, m\right.$, open, $\left.u\right]$ in $\mathrm{Tbl}_{\text {com }}$, set $v \leftarrow u$.

- Else, proceed as follows:

- If there is an entry $\left[\mathrm{com}^{\prime}, m^{\prime}\right.$, open $\left.^{\prime}, 1\right]$ in $\mathrm{Tbl}_{\text {com }}$ such that $m \neq m^{\prime}$, set $v \leftarrow 0$.

- Else, proceed as follows:

* Set $v \leftarrow \operatorname{COM}$.Verify (sid, parcom, com ${ }^{\prime}, m$, open $)$.

* Append $\left[\mathrm{com}^{\prime}, m\right.$, open, $\left.v\right]$ to $\mathrm{Tbl}_{\text {com }}$.

- Send (com.verify.end, sid, $v$ ) to $\mathcal{P}_{i}$. 
$\mathcal{F}_{\mathrm{SMT}}$ is parameterized by a message space $\mathcal{M}$ and by a leakage function $l: \mathcal{M} \rightarrow \mathbb{N}$, which leaks the message length.

1) On input (smt.send.ini, sid, $m$ ) from a party $\mathcal{T}$ :

- Abort if $\operatorname{sid} \neq\left(\mathcal{T}, \mathcal{R}\right.$, sid $\left.^{\prime}\right)$ or if $m \notin \mathcal{M}$.

- Create a fresh qid and store $(q i d, \mathcal{R}, m)$.

- Send (smt.send.sim, sid, qid, $l(m))$ to $\mathcal{S}$.

S. On input (smt.send.rep, sid, qid) from $\mathcal{S}$ :

- Abort if $(q i d, \mathcal{R}, m)$ is not stored.

- Delete the record $(q i d, \mathcal{R}, m)$.

- Send (smt.send.end, sid, $m$ ) to $\mathcal{R}$.

Fig. 9. Functionality $\mathcal{F}_{\mathrm{SMT}}$

Correctness. Correctness requires that for par $\leftarrow$ VC.Setup $\left(1^{k}, \ell\right), \mathbf{x}=(\mathbf{x}[1], \ldots, \mathbf{x}[n]) \leftarrow \mathcal{M}^{n}, r \leftarrow \mathcal{R}$, $v c \leftarrow \operatorname{VC} . \operatorname{Commit}(p a r, \mathbf{x}, r), i \leftarrow[1, n]$ and $w \leftarrow \operatorname{VC} . \operatorname{Wit}(p a r, i, \mathbf{x}, r)$, VC.Verify $(p a r, v c, \mathbf{x}[i], i, w)$ outputs 1 with probability 1 .

Hiding. The hiding property requires that any ppt adversary $\mathcal{A}$ has negligible advantage in the following game. $\mathcal{A}$ chooses a vector and sends it to the challenger. The challenger picks a random vector, commits to one of the vectors and sends the commitment to $\mathcal{A}$. $\mathcal{A}$ guesses which vector was used to compute the commitment. More formally, for $\ell$ polynomial in $k$ we require

$$
\operatorname{Pr}\left[\begin{array}{l}
\text { par } \leftarrow \text { VC.Setup }\left(1^{k}, \ell\right) ;\left(\mathbf{x}_{0}, s t\right) \leftarrow \mathcal{A}(\text { par }) ; r \leftarrow \mathcal{R} ; \\
\mathbf{x}_{1} \leftarrow \mathcal{M}^{\ell} ; b \leftarrow\{0,1\} ; v c \leftarrow \operatorname{VC} . \text { Commit }\left(\text { par }, \mathbf{x}_{b}, r\right) ; \\
b^{\prime} \leftarrow \mathcal{A}(s t, v c): b=b^{\prime} \wedge \mathbf{x}_{0} \in \mathcal{M}^{\ell}
\end{array}\right]=\frac{1}{2}+\epsilon(k)
$$

When updating a commitment, we require that $\mathcal{A}$ cannot distinguish whether a commitment $v c$ is an update of a commitment to a vector specified by the adversary or a commitment to a random vector, i.e., that

$$
\begin{aligned}
& \operatorname{Pr}\left[\begin{array}{l}
\text { par } \leftarrow \mathrm{VC} . \operatorname{Setup}\left(1^{k}, \ell\right) ;\left(\mathbf{x}, j, x^{\prime}, r, s t\right) \leftarrow \mathcal{A}(\text { par }) ; \\
r^{\prime} \leftarrow \mathcal{R} ; \mathbf{x}^{\prime} \leftarrow \mathcal{M}^{\ell} ; \\
v c \leftarrow \mathrm{VC} . \text { Commit }\left(\text { par }, \mathbf{x}^{\prime}, r^{\prime}\right): \\
1=\mathcal{A}(s t, v c) \wedge \mathbf{x} \in \mathcal{M}^{\ell} \wedge j \in[1, \ell] \wedge x^{\prime} \in \mathcal{M} \wedge r \in \mathcal{R}
\end{array}\right]= \\
& \operatorname{Pr}\left[\begin{array}{l}
p a r \leftarrow \operatorname{VC} . \operatorname{Setup}\left(1^{k}, \ell\right) ;\left(\mathbf{x}, j, x^{\prime}, r, s t\right) \leftarrow \mathcal{A}(\text { par }) ; \\
r^{\prime} \leftarrow \mathcal{R} ; \\
v c \leftarrow \operatorname{VC} . \operatorname{ComUpd}\left(\text { par }, \operatorname{VC} C \operatorname{Commit}(\text { par }, \mathbf{x}, r), j, \mathbf{x}[j], r, x^{\prime}, r^{\prime}\right): \\
1=\mathcal{A}(s t, v c) \wedge \mathbf{x} \in \mathcal{M}^{\ell} \wedge j \in[1, \ell] \wedge x^{\prime} \in \mathcal{M} \wedge r \in \mathcal{R}
\end{array}\right]
\end{aligned}
$$

Binding. The binding property requires that no adversary can output a vector commitment $v c$, a position $i \in[1, \ell]$, two values $x$ and $x^{\prime}$ and two respective witnesses $w$ and $w^{\prime}$ such that VC.Verify accepts both, i.e., for $\ell$ polynomial in $k$ :

$$
\operatorname{Pr}\left[\begin{array}{l}
\operatorname{par} \leftarrow \operatorname{VC} . \operatorname{Setup}\left(1^{k}, \ell\right) ;\left(v c, i, x, x^{\prime}, w, w^{\prime}\right) \leftarrow \mathcal{A}(\text { par }): \\
\operatorname{VC} . \operatorname{Verify}(\text { par, } v c, x, i, w)=1 \wedge x \neq x^{\prime} \wedge \\
\operatorname{VC} . \operatorname{Verify}\left(\text { par, } v c, x^{\prime}, i, w^{\prime}\right)=1 \wedge i \in[1, \ell] \wedge x, x^{\prime} \in \mathcal{M}
\end{array}\right] \leq \epsilon(k)
$$


which is parameterized by the setup algorithm VC.Setup = CRS.Setup of the VC scheme. $\mathcal{V}$ receives as input a table $\mathrm{Tbl}_{c d}$ an computes a commitment $v c$ to $\mathrm{Tbl}_{c d}$ with 0 randomness. $\mathcal{V}$ sends $\mathrm{Tbl}_{c d}$ to $\mathcal{P}$ by using the ideal functionality $\mathcal{F}_{\text {AUT }}$ for an authenticated channel, and $\mathcal{P}$ also computes a commitment $v c$ to $\mathrm{Tbl}_{c d}$ with 0 randomness.

In the read interface, $\mathcal{P}$ receives as input a position $i$ and a value $v_{r}$, along with commitments and openings $\left(\right.$ com $_{i}$, open $\left._{i}\right)$ and $\left(\right.$ com $_{r}$, open $\left._{r}\right) . \mathcal{P}$ uses the ideal functionality $\mathcal{F}_{\mathrm{ZK}}^{R_{r}}$ for zero-knowledge proofs to prove to $\mathcal{V}$ that $\mathrm{com}_{i}$ and $\mathrm{com}_{r}$ commit to $i$ and $v_{r}$ such that $v_{r}$ is the message committed at position $i$ in the commitment $v c$. This proves that $\left[i, v_{r}\right] \in$ $\mathrm{Tbl}_{c d}$.

In the write interface, $\mathcal{P}$ receives as input a position $i$ and a value $v_{w}$, along with commitments and openings $\left(\mathrm{com}_{i}\right.$, open $\left._{i}\right)$ and $\left(\right.$ com $_{w}$, open $\left._{w}\right) . \mathcal{P}$ updates the commitment $v c$ to a commitment $v c^{\prime}$ that commits to $v_{w}$ at position $i$, while other positions remain unchanged. $\mathcal{P}$ uses the functionality $\mathcal{F}_{\mathrm{ZK}}^{R_{w}}$ to prove to $\mathcal{V}$ that $\operatorname{com}_{i}$ and $\operatorname{com}_{w}$ commit to $i$ and $v_{w}$, and that $v c^{\prime}$ is an update of $v c$ where $v_{w}$ is committed in the position $i$. We note that $v c^{\prime}$ contains randomness chosen by $\mathcal{P}$ and thus, after the first execution of the write interface, the committed table will be hidden from $\mathcal{V}$.

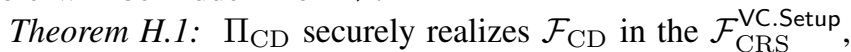
$\mathcal{F}_{\mathrm{AUT}}, \mathcal{F}_{\mathrm{ZK}}^{R_{r}}$ and $\mathcal{F}_{\mathrm{ZK}}^{R_{w}}$-hybrid model if the $\mathrm{VC}$ scheme (VC.Setup, VC.Commit, VC.Wit, VC.Verify, VC.ComUpd, VC.VerComUpd, VC.WitUpd) is hiding and binding as defined in Appendix G.

When $\mathcal{P}$ is corrupt, the binding property of the vector commitment scheme guarantees that the adversary is not able to open the vector commitment to a position and a value if that value was not previously committed at that position. When $\mathcal{V}$ is corrupt, the hiding property of the $\mathrm{VC}$ scheme guarantees that the committed vector remains hidden from $\mathcal{V}$. We analyze in detail the security of $\Pi_{\mathrm{CD}}$ in Appendix $\mathrm{I}$.

\section{APPENDIX I}

\section{SeCURity AnAlysis of CONSTRUCTION $\Pi_{\mathrm{CD}}$}

Theorem I.1: $\Pi_{\mathrm{CD}}$ securely realizes $\mathcal{F}_{\mathrm{CD}}$ in the $\mathcal{F}_{\mathrm{CRS}}^{\mathrm{VC} \text {.Setup, }}$ $\mathcal{F}_{\mathrm{AUT}}, \mathcal{F}_{\mathrm{ZK}}^{R_{r}}$ and $\mathcal{F}_{\mathrm{ZK}}^{R_{w}}$-hybrid model if the $\mathrm{VC}$ scheme (VC.Setup, VC.Commit, VC.Wit, VC.Verify, VC.ComUpd, VC.VerComUpd, VC.WitUpd) is hiding and binding as defined in Appendix G.

To prove that our construction $\Pi_{\mathrm{CD}}$ securely realizes the ideal functionality $\mathcal{F}_{\mathrm{CD}}$, we have to show that for any environment $\mathcal{Z}$ and any adversary $\mathcal{A}$ there exists a simulator $\mathcal{S}$ such that $\mathcal{Z}$ cannot distinguish whether it is interacting with $\mathcal{A}$ and the protocol in the real world or with $\mathcal{S}$ and $\mathcal{F}_{\mathrm{CD}}$. The simulator thereby plays the role of all honest parties in the real world and interacts with $\mathcal{F}_{\mathrm{CD}}$ for all corrupt parties in the ideal world.

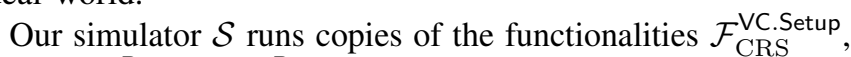
$\mathcal{F}_{\mathrm{AUT}}, \mathcal{F}_{\mathrm{ZK}}^{R_{r}}$ and $\mathcal{F}_{\mathrm{ZK}}^{R_{w}}$. When any of the copies of those functionalities aborts, $\mathcal{S}$ implicitly forwards the abortion message to the adversary if the functionality sends the abortion message to a corrupt party.
In Appendix I-A, we analyze the security of construction $\Pi_{\mathrm{CD}}$ when the prover $\mathcal{P}$ is corrupt. In Appendix I-B, we analyze the security of construction $\Pi_{\mathrm{CD}}$ when the verifier $\mathcal{V}$ is corrupt.

\section{A. Security Analysis of Construction $\Pi_{\mathrm{CD}}$ when $\mathcal{P}$ is Corrupt}

First, we describe the simulator $\mathcal{S}$ for the case in which the prover is corrupt.

Honest $\mathcal{V}$ sends $\mathbf{T b l}_{c d}$. On input (cd.setup.sim, sid, $\mathrm{Tbl}_{c d}$ ) from $\mathcal{F}_{\mathrm{CD}}, \mathcal{S}$ sets $\operatorname{sid}_{\mathrm{AUT}} \leftarrow\left(\mathcal{V}, \mathcal{P}\right.$, sid $\left.^{\prime}\right)$ and runs a copy of $\mathcal{F}_{\mathrm{AUT}}$ on input (aut.send.ini, $\operatorname{sid}_{\mathrm{AUT}}, \mathrm{Tbl}_{c d}$ ). When $\mathcal{F}_{\mathrm{AUT}}$ sends (aut.send.sim, $\operatorname{sid}_{\mathrm{AUT}}, q i d, \mathrm{~Tb}_{c d}$ ), $\mathcal{S}$ does the following:

- $\mathcal{S}$ sets $c v \leftarrow 0$.

- $\mathcal{S}$ runs a copy of $\mathcal{F}_{\mathrm{CRS}}^{\mathrm{VC} \text { Setup }}$ on input (crs.get.ini, sid). When $\mathcal{F}_{\text {CRS }}^{\mathrm{VC} \text {.Setup }}$ sends (crs.get.sim, sid, qid, par), $\mathcal{S}$

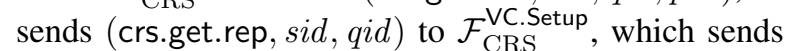
a message (crs.get.end, sid, par).

- For $i=1$ to $N, \mathcal{S}$ sets $\mathbf{x}[i] \leftarrow v$, where $[i, v] \in \mathrm{Tbl}_{c d}$. - $\mathcal{S}$ sets $r \leftarrow 0$ and computes a commitment $v c \leftarrow$ VC.Commit $(p a r, \mathbf{x}, r)$.

- $\mathcal{S}$ stores a tuple (sid, cv, par, $\mathbf{x}, v c, r)$.

$\mathcal{S}$ sends (aut.send.sim, $\operatorname{sid}_{\mathrm{AUT}}, q i d, \mathrm{Tbl}_{c d}$ ) to $\mathcal{A}$.

$\mathcal{A}$ receives $\mathbf{T b l}_{c d}$. On input (aut.send.rep, $s i d_{\mathrm{AUT}}$, qid) from the adversary $\mathcal{A}$, the simulator $\mathcal{S}$ runs the functionality $\mathcal{F}_{\text {AUT }}$ on input that message. When the functionality $\mathcal{F}_{\mathrm{AUT}}$ sends the message (aut.send.end, sid ${ }_{\mathrm{AUT}}$, $\mathrm{Tbl}_{c d}$ ), $\mathcal{S}$ sends the message (cd.setup.rep, sid) to $\mathcal{F}_{\mathrm{CD}}$ When $\mathcal{F}_{\mathrm{CD}}$ sends (cd.setup.end, sid, $\mathrm{Tbl}_{c d}$ ), $\mathcal{S}$ sends (aut.send.end, $\operatorname{sid}_{\mathrm{AUT}}, \mathrm{Tbl}_{c d}$ ) to $\mathcal{A}$.

$\mathcal{A}$ requests par. On input (crs.get.ini, sid) from $\mathcal{A}, \mathcal{S}$ runs a copy of $\mathcal{F}_{\mathrm{CRS}}^{\mathrm{VC} \text {. Setup }}$ on that input. When the copy of $\mathcal{F}_{\text {CRS }}^{\mathrm{VC} . \text { Setup }}$ sends (crs.get.sim, sid, qid, par), $\mathcal{S}$ forwards that message to $\mathcal{A}$.

$\mathcal{A}$ receives par. On input (crs.get.rep, sid, qid) from $\mathcal{A}, \mathcal{S}$ runs a copy of $\mathcal{F}_{\mathrm{CRS}}^{\mathrm{VC} \text {.Setup }}$ on that input. When the copy of $\mathcal{F}_{\mathrm{CRS}}^{\mathrm{VC} \text {.Setup }}$ sends (crs.get.end, sid, par), $\mathcal{S}$ sends (crs.get.end, sid, par) to $\mathcal{A}$.

$\mathcal{A}$ starts a read proof. On input the message (zk.prove.ini, sid, wit $t_{r}$, ins $\left._{r}\right)$ from $\mathcal{A}, \mathcal{S}$ runs a copy of $\mathcal{F}_{\mathrm{ZK}}^{R_{r}}$ on that input. When the copy of $\mathcal{F}_{\mathrm{ZK}}^{R_{r}}$ sends (zk.prove.sim, sid, qid, ins $\left._{r}\right), \mathcal{S}$ stores $\left(q i d\right.$, wit $_{r}$, ins $\left._{r}\right)$ and sends (zk.prove.sim, sid, qid, ins $s_{r}$ ) to $\mathcal{A}$.

$\mathcal{A}$ ends a read proof. On input the message (zk.prove.rep, sid, qid) from $\mathcal{A}, \mathcal{S}$ runs a copy of $\mathcal{F}_{\mathrm{ZK}}^{R_{r}}$ on that input. When the copy of $\mathcal{F}_{\mathrm{ZK}}^{R_{r}}$ outputs (zk.prove.end, sid, ins $\left._{r}\right), \mathcal{S}$ retrieves the stored tuple $\left(q i d\right.$, wit $_{r}$, ins $\left._{r}\right)$ and parses the instance ins $_{r}$ as $\left(\right.$ par $^{\prime}, v c^{\prime}$, parcom $_{i}, \operatorname{com}_{i}^{\prime}$, parcom $\left._{r}, \operatorname{com}_{r}^{\prime}, c p\right)$. The simulator $\mathcal{S}$ sets the commitments $\operatorname{com}_{i} \leftarrow\left(\operatorname{com}_{i}^{\prime}\right.$, parcom $_{i}$, COM.Verify) and $\operatorname{com}_{r}$ $\leftarrow\left(\right.$ com $_{r}^{\prime}$, parcom $_{r}$, COM.Verify). $\mathcal{S}$ sends (cd.read.ini, sid, com $_{i}, i$, open $_{i}$, com $_{r}, v_{r}$, open $\left._{r}\right)$ to the functionality $\mathcal{F}_{\mathrm{CD}}$. When $\mathcal{F}_{\mathrm{CD}}$ sends (cd.read.sim, sid, qid, $\operatorname{com}_{i}$, $\left.\mathrm{com}_{r}\right), \mathcal{S}$ does the following: 
$\Pi_{\mathrm{CD}}$ uses a vector commitment scheme (VC.Setup, VC.Commit, VC.Wit, VC.Verify, VC.ComUpd, VC.VerComUpd, VC.WitUpd) and the ideal functionalities $\mathcal{F}_{\mathrm{CRS}}^{\mathrm{VC} \text {.Setup }}, \mathcal{F}_{\mathrm{AUT}}, \mathcal{F}_{\mathrm{ZK}}^{R_{r}}$ and $\mathcal{F}_{\mathrm{ZK}}^{R_{w}}$. The table size $N_{\text {max }}$ is the maximum length of a committed vector, and the universe of values $U_{v}$ is given by the message space of the VC scheme.

1) On input (cd.setup.ini, sid, $\mathrm{Tbl}_{c d}$ ), $\mathcal{V}$ and $\mathcal{P}$ do the following:

- $\mathcal{V}$ aborts if $(s i d, c v, p a r, v c)$ is already stored.

- $\mathcal{V}$ aborts if $\operatorname{sid} \neq\left(\mathcal{P}, \mathcal{V}\right.$, sid $\left.^{\prime}\right)$, or if $\operatorname{Tbl}_{c d}$ does not consist of entries of the form $[i, v]$, or if the number of entries in $\mathrm{Tbl}_{c d}$ is not $N_{\max }$, or if, for $i=1$ to $N_{\max }, v \notin U_{v}$ for any entry $[i, v]$ in $\mathrm{Tbl}_{c d}$.

- $\mathcal{V}$ sends (crs.get.ini, sid) to $\mathcal{F}_{\mathrm{CRS}}^{\mathrm{VC} \text {.Setup }}$ and receives (crs.get.end, sid, par) from $\mathcal{F}_{\mathrm{CRS}}^{\mathrm{VC} \text {. Setup }}$. To compute par, $\mathcal{F}_{\mathrm{CRS}}^{\mathrm{VC} \text {. Setup }}$ runs VC.Setup $\left(1^{k}, N_{\max }\right)$.

- $\mathcal{V}$ sets a counter $c v \leftarrow 0$ and a vector $\mathbf{x}$ such that, for $i=1$ to $N_{\text {max }}, \mathbf{x}[i]=v$, where $[i, v] \in \mathrm{Tbl}_{c d}$. $\mathcal{V}$ sets $r \leftarrow 0$ and runs $v c \leftarrow \mathrm{VC}$.Commit $($ par, $\mathbf{x}, r)$. $\mathcal{V}$ stores $($ sid, $c v, p a r, v c)$.

- $\mathcal{V}$ sets $\operatorname{sid}_{\mathrm{AUT}} \leftarrow\left(\mathcal{V}, \mathcal{P}\right.$, sid $\left.{ }^{\prime}\right)$ and sends (aut.send.ini, $\left.\operatorname{sid}_{\mathrm{AUT}}, \mathrm{Tb}_{c d}\right)$ to the functionality $\mathcal{F}_{\mathrm{AUT}}$.

- $\mathcal{P}$ receives (aut.send.end, $\operatorname{sid}_{\mathrm{AUT}}, \mathrm{Tbl}_{c d}$ ) from $\mathcal{F}_{\mathrm{AUT}}$.

- $\mathcal{P}$ sets $\operatorname{sid} \leftarrow\left(\mathcal{P}, \mathcal{V}, s i d^{\prime}\right)$ and aborts if $(s i d, c p, p a r, v c, \mathbf{x}, r)$ is already stored.

- $\mathcal{P}$ aborts if $\mathrm{Tbl}_{c d}$ does not consist of entries of the form $[i, v]$, or if the number of entries in $\mathrm{Tbl}_{c d}$ is not $N_{\max }$, or if, for $i=1$ to $N_{\max }, v \notin U_{v}$ for any entry $[i, v]$ in $\mathrm{Tbl}_{c d}$.

- $\mathcal{P}$ sends (crs.get.ini, sid) to $\mathcal{F}_{\mathrm{CRS}}^{\mathrm{VC} \text { Setup }}$ and receives (crs.get.end, sid, par) from $\mathcal{F}_{\mathrm{CRS}}^{\mathrm{VC} \text {.Setup }}$. To compute par, $\mathcal{F}_{\mathrm{CRS}}^{\mathrm{VC} \text {. Setup }}$ runs VC.Setup $\left(1^{k}, N_{\max }\right)$.

- $\mathcal{P}$ sets a counter $c p \leftarrow 0$ and a vector $\mathbf{x}$ such that, for $i=1$ to $N_{\text {max }}, \mathbf{x}[i]=v$, where $[i, v] \in \operatorname{Tb}_{c d}$. $\mathcal{P}$ sets $r \leftarrow 0$ and runs $v c \leftarrow$ VC.Commit $($ par $, \mathbf{x}, r)$. $\mathcal{P}$ stores $(s i d, c p, p a r, v c, \mathbf{x}, r)$.

- $\mathcal{P}$ outputs (cd.setup.end, sid, $\mathrm{Tbl}_{c d}$ ).

2) On input (cd.read.ini, $\operatorname{sid}, \mathrm{com}_{i}, i$, open $_{i}, \mathrm{com}_{r}, v_{r}$, open $_{r}$ ), $\mathcal{P}$ and $\mathcal{V}$ do the following:

- $\mathcal{P}$ aborts if ( $s i d, c p, p a r, v c, \mathbf{x}, r)$ is not stored.

- $\mathcal{P}$ aborts if $i \notin\left[1, N_{\text {max }}\right]$, or if $v_{r} \notin U_{v}$, or if $\mathbf{x}[i] \neq v_{r}$.

- $\mathcal{P}$ parses com $_{i}$ as $\left(\right.$ comm $_{i}^{\prime}$, parcom $_{i}$, COM.Verify $\left._{i}\right)$.

- $\mathcal{P}$ parses com $_{r}$ as $\left(\right.$ com $_{r}^{\prime}$, parcom $_{r}$, COM.Verify $\left.{ }_{r}\right)$.

- $\mathcal{P}$ aborts if COM.Verify ${ }_{i}$ or COM.Verify ${ }_{r}$ are not ppt algorithms.

- $\mathcal{P}$ aborts if $1 \neq$ COM.Verify $_{i}\left(\right.$ parcom $_{i}$, com $_{i}, i$, open $\left._{i}\right)$.

- $\mathcal{P}$ aborts if $1 \neq$ COM.Verify $_{r}\left(\right.$ parcom $_{r}$, com $_{r}, v_{r}$, open $\left._{r}\right)$.

- If $(\operatorname{sid}, i, w)$ is not stored, $\mathcal{P}$ runs $w \leftarrow \operatorname{VC}$.Wit $($ par, $i, \mathbf{x}, r)$ and stores $(\operatorname{sid}, i, w)$.

- $\mathcal{P}$ sets wit $_{r} \leftarrow\left(w, i\right.$, open $_{i}, v_{r}$, open $\left._{r}\right)$.

- $\mathcal{P}$ sets ins $_{r} \leftarrow\left(\right.$ par, vc, parcom $_{i}$, com $_{i}^{\prime}$, parcom $_{r}$, com $\left._{r}^{\prime}, c p\right)$.

- $\mathcal{P}$ sends (zk.prove.ini, sid, wit ${ }_{r}$, ins $_{r}$ ) to $\mathcal{F}_{\mathrm{ZK}}^{R_{r}}$, where the relation $R_{r}$ is

$$
\begin{aligned}
R_{r}= & \left\{\left(\text { wit }_{r}, \text { ins }_{r}\right):\right. \\
& 1=\text { COM.Verify }_{i}\left(\text { parcom }_{i}, \text { com }_{i}^{\prime}, i, \text { open }_{i}\right) \wedge \\
& 1=\text { COM.Verify }_{r}\left(\text { parcom }_{r}, \text { com }_{r}^{\prime}, v_{r}, \text { open }_{r}\right) \wedge \\
& \left.1=\operatorname{VC} . \operatorname{Verify}\left(\text { par }, v c, v_{r}, i, w\right)\right\}
\end{aligned}
$$

In equation 20, $\mathcal{P}$ proves that $\operatorname{com}_{i}$ is a commitment to $i$ with opening $\operatorname{open}_{i}$. Similarly, in equation $21, \mathcal{P}$ proves that $\mathrm{com}_{r}$ is a commitment to $v_{r}$ with opening open $_{r}$. In equation 22, $\mathcal{P}$ proves that $v_{r}$ is stored in the position $i$ of the vector commitment $v c$.

- $\mathcal{V}$ receives (zk.prove.end, sid, ins $s_{r}$ ) from $\mathcal{F}_{\mathrm{ZK}}^{R_{r}}$.

- $\mathcal{V}$ aborts if $(s i d, c v, p a r, v c)$ is not stored.

- $\mathcal{V}$ parses ins $_{r}$ as $\left(\right.$ par $^{\prime}, v^{\prime}$, parcom $_{i}$, com $_{i}^{\prime}$, parcom $_{r}$, com $\left._{r}^{\prime}, c p\right)$.

- $\mathcal{V}$ aborts if $c p \neq c v$, or if $p a r^{\prime} \neq$ par, or if $v c^{\prime} \neq v c$.

- $\mathcal{V}$ sets $\operatorname{com}_{i} \leftarrow\left(\operatorname{com}_{i}^{\prime}\right.$, parcom $_{i}$, COM.Verify $\left._{i}\right)$ and $\operatorname{com}_{r} \leftarrow\left(\operatorname{com}_{r}^{\prime}\right.$, parcom $_{r}$, COM.Verify $\left._{r}\right)$.

- $\mathcal{V}$ outputs (cd.read.end, sid, $\operatorname{com}_{i}, \operatorname{com}_{r}$ ).

Fig. 11. Construction $\Pi_{\mathrm{CD}}$ : interfaces cd.setup and cd.read 
3. On input (cd.write.ini, sid, $\operatorname{com}_{i}, i$, open $_{i}, \operatorname{com}_{w}, v_{w}$, open $_{w}$ ), $\mathcal{P}$ and $\mathcal{V}$ do the following:

- $\mathcal{P}$ aborts if ( $s i d, c p, p a r, v c, \mathbf{x}, r)$ is not stored.

- $\mathcal{P}$ aborts if $i \notin\left[1, N_{\max }\right]$, or if $v_{w} \notin U_{v}$.

- $\mathcal{P}$ parses com $_{i}$ as $\left(\right.$ com $_{i}^{\prime}$, parcom $_{i}$, COM.Verify $\left._{i}\right)$.

- $\mathcal{P}$ parses $\operatorname{com}_{w}$ as $\left(\right.$ com $_{w}^{\prime}$, parcom $_{w}$, COM.Verify $\left._{w}\right)$.

- $\mathcal{P}$ aborts if COM.Verify ${ }_{i}$ or COM.Verify ${ }_{w}$ are not ppt algorithms.

- $\mathcal{P}$ aborts if $1 \neq$ COM.Verify $_{i}\left(\right.$ parcom $_{i}$, com $_{i}, i$, open $\left._{i}\right)$.

- $\mathcal{P}$ aborts if $1 \neq$ COM.Verify $_{w}\left(\right.$ parcom $_{w}$, com $_{w}, v_{w}$, open $\left._{w}\right)$.

- If $($ sid, $i, w)$ is not stored, $\mathcal{P}$ runs $w \leftarrow \operatorname{VC}$.Wit $($ par, $i, \mathbf{x}, r)$ and stores $($ sid, $i, w)$.

- $\mathcal{P}$ picks random $r^{\prime} \leftarrow \mathcal{R}$ and runs $v c^{\prime} \leftarrow \operatorname{VC}$. ComUpd $\left(\right.$ par, $\left.v c, i, v_{r}, r, v_{w}, r^{\prime}\right)$, where $v_{r} \leftarrow \mathbf{x}[i]$.

- $\mathcal{P}$ sets $c p^{\prime} \leftarrow c p+1$.

- $\mathcal{P}$ sets wit $_{w} \leftarrow\left(w, i\right.$, open $_{i}, v_{r}, v_{w}$, open $\left._{w}, r, r^{\prime}\right)$.

- $\mathcal{P}$ sets ins $_{w} \leftarrow\left(\right.$ par, $v c, v c^{\prime}$, parcom $_{i}$, com $_{i}^{\prime}$, parcom $_{w}$, com $\left._{w}^{\prime}, c p^{\prime}\right)$.

- $\mathcal{P}$ sets $\mathbf{x}^{\prime} \leftarrow \mathbf{x}$ and $\mathbf{x}^{\prime}[i] \leftarrow v_{w}$.

- $\mathcal{P}$ replaces the stored tuple (sid, $c p$, par, $v c, \mathbf{x}, r)$ by $\left(s i d, c p^{\prime}, p a r, v c^{\prime}, \mathbf{x}^{\prime}, r^{\prime}\right)$.

- For $j=1$ to $N_{\max }$, if $(\operatorname{sid}, j, w)$ is stored, $\mathcal{P}$ runs $w^{\prime} \leftarrow \operatorname{VC}$.WitUpd $\left(p a r, w, j, i, x, r, x^{\prime}, r^{\prime}\right)$ and replaces $(\operatorname{sid}, j$, $w)$ by $\left(s i d, j, w^{\prime}\right)$.

- $\mathcal{P}$ sends (zk.prove.ini, sid, wit ${ }_{w}$, ins $s_{w}$ ) to $\mathcal{F}_{\mathrm{ZK}}^{R_{w}}$, where $R_{w}$ is

$$
\begin{aligned}
R_{w}= & \left\{\left(\text { wit }_{w}, \text { ins }_{w}\right):\right. \\
& 1=\text { COM.Verify }_{i}\left(\text { parcom }_{i}, \text { com }_{i}, i, \text { open }_{i}\right) \wedge \\
& 1=\text { COM.Verify }_{w}\left(\text { parcom }_{w}, \text { com }_{w}, v_{w}, \text { open }_{w}\right) \wedge \\
& \left.1=\text { VC.VerComUpd }\left(\text { par }, v c, v c^{\prime}, w, i, v_{r}, r, v_{w}, r^{\prime}\right)\right\}
\end{aligned}
$$

In equation 23 and equation 24, the prover proves that $\operatorname{com}_{i}$ and $\operatorname{com}_{w}$ are commitments to $i$ and $v_{w}$ respectively. In equation 25, the prover proves that $v_{r}$ is stored in the position $i$ in the vector commitment $v c$, and that $v c^{\prime}$ is a vector commitment that stores the same values as $v c$, except that it stores $v_{w}$ in the position $i$ and that its random value is $r^{\prime}$ instead of $r$.

- $\mathcal{V}$ receives (zk.prove.end, sid, ins $s_{w}$ ) from $\mathcal{F}_{\mathrm{ZK}}^{R_{w}}$.

- $\mathcal{V}$ aborts if $(s i d, c v, p a r, v c)$ is not stored.

- $\mathcal{V}$ parses ins $_{w}$ as $\left(p \hat{a} r, \hat{v} c, v c^{\prime}\right.$, parcom $\left._{i}, \operatorname{com}_{i}^{\prime}, \operatorname{parcom}_{w}, \operatorname{com}_{w}^{\prime}, c p\right)$.

- $\mathcal{V}$ aborts if $c p \neq c v+1$, or if $p \hat{a} r \neq p a r$, or if $\hat{v} c \neq v c$.

- $\mathcal{V}$ sets $c v^{\prime} \leftarrow c v+1$ and replaces $(s i d, c v, p a r, v c)$ by $\left(s i d, c v^{\prime}, p a r, v c^{\prime}\right)$.

- $\mathcal{V}$ sets $\operatorname{com}_{i} \leftarrow\left(\operatorname{com}_{i}^{\prime}\right.$, parcom $_{i}$, COM.Verify $\left._{i}\right)$ and $\operatorname{com}_{w} \leftarrow\left(\operatorname{com}_{w}^{\prime}\right.$, parcom $_{w}$, COM.Verify $\left._{w}\right)$.

- $\mathcal{V}$ outputs (cd.write.end, sid, $\operatorname{com}_{i}, \operatorname{com}_{w}$ ).

Fig. 12. Construction $\Pi_{\mathrm{CD}}$ : interface cd.write

- $\mathcal{S}$ retrieves the stored tuple $(s i d, c v, p a r, \mathbf{x}, v c, r)$. If $c p \neq c v$, or if $p a r^{\prime} \neq p a r$, or if $v c^{\prime} \neq v c, \mathcal{S}$ sends $\mathcal{F}_{\mathrm{CD}}$ a message that makes $\mathcal{F}_{\mathrm{CD}}$ abort.

- Else, $\mathcal{S}$ retrieves the stored tuple $\left(q i d, w_{i} t_{r}, i n s_{r}\right)$ and parses wit $_{r}$ as $\left(w, i\right.$, open $_{i}, v_{r}$, open $\left._{r}\right)$. $\mathcal{S}$ retrieves the stored tuple (sid, $c v, p a r, \mathbf{x}, v c, r)$. If $\mathbf{x}[i] \neq v_{r}, \mathcal{S}$ outputs failure.

- Else, $\mathcal{S}$ sends (cd.read.rep, sid, qid) to $\mathcal{F}_{\mathrm{CD}}$.

$\mathcal{A}$ starts a write proof. On input the message (zk.prove.ini, sid, wit ${ }_{w}$, ins $\left._{w}\right)$ to $\mathcal{F}_{\mathrm{ZK}}^{R_{w}}$ from $\mathcal{A}, \mathcal{S}$ runs a copy of $\mathcal{F}_{\mathrm{ZK}}^{R_{w}}$ on that input. When the copy of $\mathcal{F}_{\mathrm{ZK}}^{R_{w}}$ sends (zk.prove.sim, sid, qid, ins $s_{w}$ ), $\mathcal{S}$ stores ( $q i d$, wit $_{w}$, ins $_{w}$ ) and sends (zk.prove.sim, sid, qid, ins $s_{w}$ ) to $\mathcal{A}$.

$\mathcal{A}$ ends a write proof. On input the message (zk.prove.rep, sid, qid) from $\mathcal{A}, \mathcal{S}$ runs a copy of $\mathcal{F}_{\mathrm{ZK}}^{R_{w}}$ on that input. When the copy of $\mathcal{F}_{\mathrm{ZK}}^{R_{w}}$ outputs (zk.prove.end, sid, ins $\left._{w}\right), \mathcal{S}$ retrieves the stored tuple $\left(q i d\right.$, wit $_{w}$, ins $\left._{w}\right)$ and parses the instance $i_{n} s_{w}$ as $\left(p \hat{a} r, \hat{v} c, v c^{\prime}\right.$, parcom $_{i}$, $\left.\operatorname{com}_{i}^{\prime}, \operatorname{parcom}_{w}, \operatorname{com}_{w}^{\prime}, c p\right)$. $\mathcal{S}$ sets $\operatorname{com}_{i} \leftarrow\left(\operatorname{com}_{i}^{\prime}\right.$, parcom $_{i}$, COM.Verify $)$ and $\operatorname{com}_{w} \leftarrow\left(\right.$ com $_{w}^{\prime}$, parcom $_{w}$, COM.Verify). The simulator $\mathcal{S}$ sends (cd.write.ini, sid, com $_{i}, i$, open $_{i}, \operatorname{com}_{w}, v_{w}$, open $\left._{w}\right)$ to $\mathcal{F}_{\mathrm{CD}}$. When $\mathcal{F}_{\mathrm{CD}}$ sends (cd.write.sim, sid, qid, $\operatorname{com}_{i}, \operatorname{com}_{w}$ ), $\mathcal{S}$ does the following:

- $\mathcal{S}$ retrieves the stored tuple $(s i d, c v, p a r, \mathbf{x}, v c, r)$. If $c p \neq c v+1$, or if $p \hat{a} r \neq p a r$, or if $\hat{v c} \neq v c, \mathcal{S}$ sends $\mathcal{F}_{\mathrm{CD}}$ a message that makes $\mathcal{F}_{\mathrm{CD}}$ abort.

- Else, $\mathcal{S}$ retrieves the stored tuple (qid, wit w $_{w}$, ins $_{w}$ ) and parses wit $_{w}$ as $\left(w, i\right.$, open $_{i}, v_{r}, v_{w}$, open $\left._{w}, r, r^{\prime}\right)$. $\mathcal{S}$ retrieves the stored tuple (sid, $c v, p a r, \mathbf{x}, v c, r$ ). If 
$\mathbf{x}[i] \neq v_{r}, \mathcal{S}$ outputs failure.

- Else, $\mathcal{S}$ sets $c v^{\prime} \leftarrow c v+1, \mathbf{x}^{\prime} \leftarrow \mathbf{x}$ and $\mathbf{x}^{\prime}[i] \leftarrow v_{w}$. $\mathcal{S}$ replaces (sid, cv, par, $\mathbf{x}, v c, r)$ by (sid, $c v^{\prime}, p a r, \mathbf{x}^{\prime}$, $\left.v c^{\prime}, r^{\prime}\right) . \mathcal{S}$ sends (cd.write.rep, sid, qid) to $\mathcal{F}_{\mathrm{CD}}$.

Theorem I.2: When the prover $\mathcal{P}$ is corrupt, the construction

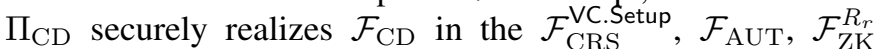
and $\mathcal{F}_{\mathrm{ZK}}^{R_{w}}$-hybrid model if the vector commitment scheme (VC.Setup, VC.Commit, VC.Wit, VC.Verify, VC.ComUpd, VC.VerComUpd, VC.WitUpd) is binding as defined in Appendix G.

Proof of Theorem I.2. We show by means of a series of hybrid games that the environment $\mathcal{Z}$ cannot distinguish between the ensemble $\mathrm{REAL}_{\mathrm{CD}, \mathcal{A}, \mathcal{Z}}$ and the ensemble $\operatorname{IDEAL}_{\mathcal{F}_{\mathrm{CD}}, \mathcal{S}, \mathcal{Z}}$ with non-negligible probability. We denote by $\operatorname{Pr}[$ Game $i]$ the probability that the environment distinguishes Game $i$ from the real-world protocol.

Game 0: This game corresponds to the execution of the realworld protocol. Therefore, Pr $[$ Game 0] $=0$.

Game 1: Game 1 follows Game 0, except that Game 1 stores a tuple (sid, $c v, p a r, \mathbf{x}, v c, r)$. Game 1 updates the values $(c v, \mathbf{x}, v c, r)$ of that tuple each time the adversary sends a valid write proof. These changes do not alter the view of the environment. Therefore, $\mid \operatorname{Pr}[$ Game 1] $-\operatorname{Pr}[$ Game 0]| $=0$.

Game 2: Game 2 follows Game 1, except that, when the adversary sends a valid read proof with witness wit $_{r}$ and instance $i n s_{r}$, Game 2 outputs failure if the values $i$ and $v_{r}$ in the witness are such that $\mathbf{x}[i] \neq v_{r}$, where $\mathbf{x}[i]$ is in the stored tuple (sid, cv, par, $\mathbf{x}, v c, r)$. Similarly, when the adversary sends a valid write proof with witness wit $_{w}$ and instance $i n s_{w}$, Game 2 outputs failure if the values $i$ and $v_{r}$ in the witness are such that $\mathbf{x}[i] \neq v_{r}$, where $\mathbf{x}[i]$ is in the stored tuple ( $s i d, c v, p a r, \mathbf{x}, v c, r)$. The probability that Game 2 outputs failure is bound by the following claim.

Theorem I.3: Under the binding property of the vector commitment scheme, we have that $\mid \operatorname{Pr}$ [Game 2] $\operatorname{Pr}[$ Game 1$] \mid \leq \operatorname{Adv}_{\mathcal{A}}^{\text {bin }-\mathrm{vc}}$.

Proof of Theorem I.3. We construct an algorithm $B$ that, given an adversary that makes Game 2 fail with non-negligible probability, breaks the binding property of the vector commitment scheme with non-negligible probability. $B$ behaves as Game 2 with the following three modifications:

- When the challenger sends the parameters par, $B$ stores par as common reference string in the copy of $\mathcal{F}_{\text {CRS }}^{\mathrm{VC} \text {.Setup }}$.

- When the adversary sends a valid read proof with witness wit $_{r}=\left(w, i\right.$, open $_{i}, v_{r}$, open $\left._{r}\right)$ and instance ins $_{r}=\left(\right.$ par $^{\prime}, v^{\prime}$, parcom $_{i}$, com $_{i}^{\prime}$, parcom $_{r}$, com $\left._{r}^{\prime}, c p\right)$ such that the values $i$ and $v_{r}$ in the witness fulfill $\mathbf{x}[i]$ $\neq v_{r}$, where $\mathbf{x}[i]$ is in the stored tuple (sid, cv,par, $\mathbf{x}, v c, r), B$ runs $w^{\prime} \leftarrow \operatorname{VC}$.Wit $(p a r, i, \mathbf{x}, r)$ and sends (vc, $\left.i, v_{r}, \mathbf{x}[i], w, w^{\prime}\right)$ to the challenger.

- When the adversary sends a valid write proof with the witness wit $_{w}=\left(w, i\right.$, open $_{i}, v_{r}, v_{w}$, open $\left._{w}, r, r^{\prime}\right)$ and the instance $i n s_{w}=\left(p \hat{a r}, \hat{v c}, v c^{\prime}, \operatorname{parcom}_{i}, \operatorname{com}_{i}^{\prime}\right.$, $\left.\operatorname{parcom}_{w}, \operatorname{com}_{w}^{\prime}, c p\right)$ such that the values $i$ and $v_{r}$ in the witness fulfill $\mathbf{x}[i] \neq v_{r}$, where $\mathbf{x}[i]$ is in the stored tuple $(c v, \mathbf{x}, v c, r, p a r), B$ runs $w^{\prime} \leftarrow$ VC.Wit $(p a r, i, \mathbf{x}, r)$ and sends $\left(v c, i, v_{r}, \mathbf{x}[i], w, w^{\prime}\right)$ to the challenger.

This concludes the proof of Theorem I.3.

The distribution of Game 2 is identical to our simulation. This concludes the proof of Theorem I.2.

\section{B. Security Analysis of Construction $\Pi_{\mathrm{CD}}$ when $\mathcal{V}$ is Corrupt}

We describe the simulator $\mathcal{S}$ for the case in which the verifier is corrupt.

$\mathcal{A}$ requests par. On input (crs.get.ini, sid) from $\mathcal{A}, \mathcal{S}$ runs the copy of $\mathcal{F}_{\mathrm{CRS}}^{\mathrm{VC} \text {.Setup }}$ on that input. When the copy of $\mathcal{F}_{\text {CRS }}^{\mathrm{VC} \text {.Setup }}$ sends (crs.get.sim, sid, qid,par), $\mathcal{S}$ forwards that message to $\mathcal{A}$.

$\mathcal{A}$ receives par. On input (crs.get.rep, sid, qid) from $\mathcal{A}, \mathcal{S}$

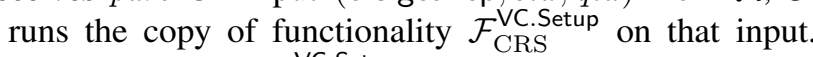
When the copy of $\mathcal{F}_{\mathrm{CRS}}^{\mathrm{VC} \text {.Setup }}$ sends (crs.get.end, sid, par), $\mathcal{S}$ forwards that message to $\mathcal{A}$.

$\mathcal{A}$ sends $\mathbf{T b l}_{c d}$. On input the message (aut.send.ini, sid ${ }_{\mathrm{AUT}}$, $\mathrm{Tbl}_{c d}$ ) from $\mathcal{A}$, the simulator $\mathcal{S}$ checks that $\operatorname{sid}_{\mathrm{AUT}}=$ $\left(\mathcal{V}, \mathcal{P}\right.$, sid $\left.^{\prime}\right)$ and runs a copy of $\mathcal{F}_{\mathrm{AUT}}$ on that input. When $\mathcal{F}_{\mathrm{AUT}}$ sends (aut.send.sim, sid $\mathrm{AUT}_{\mathrm{AT}}$, qid, $\mathrm{Tbl}_{c d}$ ), $\mathcal{S}$ sends (cd.setup.ini, sid, $\mathrm{Tbl}_{c d}$ ) to the functionality $\mathcal{F}_{\mathrm{CD}}$. When $\mathcal{F}_{\mathrm{CD}}$ sends (cd.setup.sim, sid, $\mathrm{Tbl}_{c d}$ ), $\mathcal{S}$ sends the message (aut.send.sim, sid ${ }_{\mathrm{AUT}}, q i d, \mathrm{Tbl}_{c d}$ ) to $\mathcal{A}$.

Honest $\mathcal{P}$ receives $\mathbf{T b l}_{c d}$. On input (aut.send.rep, sid $_{\mathrm{AUT}}$, qid) from $\mathcal{A}, \mathcal{S}$ runs the copy of $\mathcal{F}_{\mathrm{AUT}}$ on that input. When $\mathcal{F}_{\mathrm{AUT}}$ sends (aut.send.end, sid $\mathrm{AUT}_{\mathrm{AU}}, \mathrm{Tbl}_{c d}$ ), $\mathcal{S}$ does the following:

- $\mathcal{S}$ sets $c p \leftarrow 0$.

- For $i=1$ to $N, \mathcal{S}$ sets $\mathbf{x}[i] \leftarrow v$, where $[i, v] \in \mathrm{Tbl}_{c d}$.

- $\mathcal{S}$ sets $r \leftarrow 0$ and computes a commitment $v c \leftarrow$ VC.Commit $($ par, $\mathbf{x}, r)$.

- $\mathcal{S}$ runs a copy of $\mathcal{F}_{\mathrm{CRS}}^{\mathrm{VC} \text {.Setup }}$ on input (crs.get.ini, sid). When $\mathcal{F}_{\text {CRS }}^{\mathrm{VC} \text {.Setup }}$ sends (crs.get.sim, sid, qid, par), $\mathcal{S}$

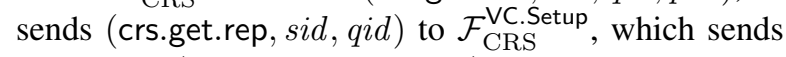
a message (crs.get.end, sid, par).

- $\mathcal{S}$ stores a tuple (sid, cp, par, vc).

$\mathcal{S}$ sends (cd.setup.rep, sid) to $\mathcal{F}_{\mathrm{CD}}$.

Honest $\mathcal{P}$ starts a read proof. On input (cd.read.sim, sid, qid, com $_{i}$, com $_{r}$ ) from $\mathcal{F}_{\mathrm{CD}}, \mathcal{S}$ does the following:

- $\mathcal{S}$ retrieves the stored tuple (sid, cp, par, vc).

- The simulator $\mathcal{S}$ parses $\operatorname{com}_{i}$ as the tuple $\left(\mathrm{com}_{i}^{\prime}\right.$, parcom $_{i}$, COM.Verify) and com $_{r}$ as the tuple $\left(\mathrm{com}_{r}^{\prime}\right.$, parcom $_{r}$, COM.Verify ${ }_{r}$ ).

- The simulator $\mathcal{S}$ sets ins $s_{r}$ as (par, vc, parcom $_{i}$, com $_{i}^{\prime}$, parcom $_{r}$, com $\left._{r}^{\prime}, c p\right)$ and stores $\left(q i d, i n s_{r}\right)$.

- $\mathcal{S}$ sends (zk.prove.sim, sid, qid, ins $s_{r}$ ) to $\mathcal{A}$.

$\mathcal{A}$ receives a read proof. On input from the adversary $\mathcal{A}$ the message (zk.prove.rep, sid, qid), the simulator $\mathcal{S}$ does the following: 
- $\mathcal{S}$ sends an abortion message to $\mathcal{A}$ if $\left(q i d\right.$, ins $\left._{r}\right)$ is not stored.

- $\mathcal{S}$ sends (cd.write.rep, sid, qid) to $\mathcal{F}_{\mathrm{CD}}$ and receives (cd.read.end, sid, $\operatorname{com}_{i}, \operatorname{com}_{r}$ ) from $\mathcal{F}_{\mathrm{CD}}$.

- $\mathcal{S}$ deletes the record $\left(q i d, i n s_{r}\right)$.

- $\mathcal{S}$ sends (zk.prove.end, sid, ins $s_{r}$ ) to $\mathcal{A}$.

Honest $\mathcal{P}$ starts a write proof. On input (cd.write.sim, sid, qid, $\operatorname{com}_{i}, \operatorname{com}_{w}$ ) from $\mathcal{F}_{\mathrm{CD}}, \mathcal{S}$ does the following:

- $\mathcal{S}$ retrieves the stored tuple (sid, $c p, p a r, v c)$.

- $\mathcal{S}$ sets $c p^{\prime} \leftarrow c p+1$.

- $\mathcal{S}$ picks a random vector $\mathrm{x} \leftarrow \mathcal{M}^{N_{\max }}$ and random $r \leftarrow \mathcal{R}$.

- The simulator $\mathcal{S}$ computes a vector commitment $v c^{\prime} \leftarrow$ VC.Commit $($ par, $\mathbf{x}, r)$.

- The simulator $\mathcal{S}$ parses $\mathrm{com}_{i}$ as the tuple $\left(\mathrm{com}_{i}^{\prime}\right.$, parcom $_{i}$, COM.Verify) and $\operatorname{com}_{w}$ as the tuple $\left(\mathrm{com}_{w}^{\prime}\right.$, parcom $_{w}$, COM.Verify ${ }_{w}$ ).

- $\mathcal{S}$ sets the instance ins $_{w}$ as (par, $v c, v c^{\prime}$, parcom $_{i}$, $\left.\operatorname{com}_{i}^{\prime}, \operatorname{parcom}_{w}, \operatorname{com}_{w}^{\prime}, c p^{\prime}\right)$ and stores $\left(q i d\right.$, ins $\left._{w}\right)$.

- $\mathcal{S}$ sends (zk.prove.sim, sid, qid, ins $s_{w}$ ) to $\mathcal{A}$.

$\mathcal{A}$ receives write proof. On input the message (zk.prove.rep, sid, qid) from $\mathcal{A}, \mathcal{S}$ does the following:

- $\mathcal{S}$ sends an abortion message to $\mathcal{A}$ if $\left(q i d, i n s_{w}\right)$ is not stored.

- $\mathcal{S}$ sends (cd.write.rep, sid, qid) to $\mathcal{F}_{\mathrm{CD}}$ and receives (cd.write.end, sid, $\operatorname{com}_{i}, \operatorname{com}_{w}$ ) from $\mathcal{F}_{\mathrm{CD}}$.

- The simulator $\mathcal{S}$ parses $i n s_{w}$ as $\left(\right.$ par, $v c, v c^{\prime}$, parcom $_{i}$, $\left.\operatorname{com}_{i}^{\prime}, \operatorname{parcom}_{w}, \operatorname{com}_{w}^{\prime}, c p^{\prime}\right) . \mathcal{S}$ replaces the stored tuple $(s i d, c p, p a r, v c)$ by $\left(s i d, c p^{\prime}, p a r, v c^{\prime}\right)$.

- $\mathcal{S}$ deletes the record (qid, ins $s_{w}$ ).

- $\mathcal{S}$ sends (zk.prove.end, sid, ins $_{w}$ ) to $\mathcal{A}$.

Theorem I.4: When the verifier $\mathcal{V}$ is corrupt, the construction $\Pi_{\mathrm{CD}}$ securely realizes $\mathcal{F}_{\mathrm{CD}}$ in the $\mathcal{F}_{\mathrm{CRS}}^{\mathrm{VC} \text {.Setup }}, \mathcal{F}_{\mathrm{AUT}}, \mathcal{F}_{\mathrm{ZK}}^{R_{r}}$ and $\mathcal{F}_{\mathrm{ZK}}^{R_{w}}$-hybrid model if the vector commitment scheme (VC.Setup, VC.Commit, VC.Wit, VC.Verify, VC.ComUpd, VC.VerComUpd, VC.WitUpd) is hiding as defined in Appendix G.

Proof of Theorem I.4. We show by means of a series of hybrid games that the environment $\mathcal{Z}$ cannot distinguish between the ensemble $\mathrm{REAL}_{\mathrm{CD}, \mathcal{A}, \mathcal{Z}}$ and the ensemble $\operatorname{IDEAL}_{\mathcal{F}_{\mathrm{CD}}, \mathcal{S}, \mathcal{Z}}$ with non-negligible probability. We denote by $\operatorname{Pr}[$ Game $i]$ the probability that the environment distinguishes Game $i$ from the real-world protocol.

Game 0: This game corresponds to the execution of the realworld protocol. Therefore, $\operatorname{Pr}[$ Game 0] $=0$.

Game 1: Game 1 follows Game 0, except that Game 1 runs an initialization phase and stores a tuple (sid, $c p, p a r, v c)$. Game 1 updates the values $(c p, v c)$ of that tuple each time the honest prover sends a write proof. These changes do not alter the view of the environment. Therefore, $\mid \operatorname{Pr}[$ Game 1] $-\operatorname{Pr}$ [Game 0] $\mid=0$.

Game 2: Game 2 follows Game 1, except that, when the honest prover sends a read proof, Game 2 does not run $\mathcal{F}_{\mathrm{ZK}}^{R_{r}}$ to compute the messages (zk.prove.sim, sid, qid, ins $s_{r}$ ) and (zk.prove.end, sid, ins $s_{r}$ ). Instead, Game 2 creates the messages directly by using the instance $i n s_{r}$, i.e., without knowledge of the witness wit $t_{r}$. Similarly, when the honest prover sends a write proof, Game 2 does not run $\mathcal{F}_{\mathrm{ZK}}^{R_{w}}$ to compute the messages (zk.prove.sim, sid, qid, ins $s_{w}$ ) and (zk.prove.end, sid, ins $_{w}$ ). Instead, Game 2 creates the messages directly by using the instance $i n s_{w}$, i.e., without knowledge of the witness wit $_{w}$. These changes do not alter the view of the environment. Therefore, $\mid \operatorname{Pr}[$ Game 2] $-\operatorname{Pr}[$ Game 1] $\mid=0$.

Game 3: Game 3 follows Game 2, except that, when the honest prover sends a read proof with instance $i n s_{r}$, Game 3 replaces the vector commitment $v c$ in $i n s_{r}$ by the vector commitment to a random vector that is in the stored tuple $(s i d, c p, p a r, v c)$. Similarly, when the adversary sends a valid write proof with instance $i n s_{w}$, Game 3 replaces the vector commitment $v c$ by the vector commitment to a random vector that is in the stored tuple (sid, $c p, p a r, v c)$, and $v c^{\prime}$ by a new vector commitment to a random vector. The probability that Game 2 and Game 3 are distinguished by the environment is bound by the following claim.

Theorem I.5: Let $M$ be the number of write proofs sent by the honest prover. Under the hiding property of the vector commitment scheme, $\mid \operatorname{Pr}[$ Game 3] $-\operatorname{Pr}[$ Game 2] $\mid \leq$ $M \cdot \mathrm{Adv}_{\mathcal{A}}^{\mathrm{hid}-\mathrm{vc}}$.

Proof of Theorem I.5. We define a sequence of games. In Game 2.i, for the last $i$ write proofs sent by the honest prover, the vector commitment $v c^{\prime}$ is replaced by a vector commitment to a random vector. Therefore, Game 2.0 corresponds to Game 2, while Game 2.M corresponds to Game 3.

We construct an algorithm $B$ that, given an adversary that distinguishes Game 2.i from Game 2. $(i+1)$ with non-negligible probability, breaks the hiding property of the vector commitment scheme with non-negligible probability. $B$ works as follows. When the challenger sends the parameters par, $B$ stores par as common reference string in the copy of $\mathcal{F}_{\mathrm{CRS}}^{\mathrm{VC} \text {.Setup }} B$ replaces the vector commitment $v c^{\prime}$ by a vector commitment to a random vector in the last $i+1$ proofs sent by the honest prover. (We note that $v c^{\prime}$ in a write proof becomes $v c$ in the next write proof). For the proof $i, B$ sends to the challenger a message $(\mathbf{x}, j, x, r)$, where $\mathbf{x}$ and $r$ are the vector and random values used to compute $v c$ and $j$ and $x^{\prime}$ are the position and the value that need to be updated to compute $v c^{\prime}$. The challenger sends back a commitment $v c^{\prime}$. As can be seen, if $v c^{\prime}$ is a commitment to a random vector, the situation corresponds to Game 2. $(i+1)$, while otherwise the situation corresponds to Game 2.i. Therefore, if the adversary distinguishes Game $2 . i$ from Game 2. $(i+1)$ with non-negligible probability, $B$ can use the adversary's guess to break the hiding property of the commitment scheme. This concludes the proof of Theorem I.5.

The distribution of Game 3 is identical to our simulation. This 
concludes the proof of Theorem I.4.

\section{APPENDIX $\mathbf{J}$ \\ SECURITy of The VC SCHEME From the DHE ASSUMPTION}

Theorem J.1: This vector commitment scheme is correct, hiding, and binding under as defined in Appendix G under the $\ell$-DHE assumption.

Correctness can be checked as follows.

$$
\begin{aligned}
e\left(v c, \tilde{g}_{i}\right) / e(w, \tilde{g}) & =\frac{e\left(g^{r+\sum_{j=1}^{n} \mathbf{x}[j]\left(\alpha^{\ell+1-j}\right)}, \tilde{g}^{\left(\alpha^{i}\right)}\right)}{e\left(g^{\left(r\left(\alpha^{i}\right)+\sum_{j=1, j \neq i}^{n} \mathbf{x}[j]\left(\alpha^{\ell+1-j+i}\right)\right)}, \tilde{g}\right)} \\
& =\frac{e\left(g^{r\left(\alpha^{i}\right)+\sum_{j=1}^{n} \mathbf{x}[j]\left(\alpha^{\ell+1-j+i}\right)}, \tilde{g}\right)}{e\left(g^{\left(r\left(\alpha^{i}\right)+\sum_{j=1, j \neq i}^{n} \mathbf{x}[j]\left(\alpha^{\ell+1-j+i}\right)\right)}, \tilde{g}\right)} \\
& =e(g, \tilde{g})^{\mathbf{x}[i]\left(\alpha^{\ell+1}\right)} \\
& =e\left(g_{1}, \tilde{g}_{\ell}\right)^{\mathbf{x}[i]} .
\end{aligned}
$$

This vector commitment scheme fulfills the hiding property in an information-theoretic way.

We show that this vector commitment scheme fulfills the binding property under the $\ell$-DHE assumption. Given an adversary $\mathcal{A}$ that breaks the binding property with nonnegligible probability $\nu$, we construct an algorithm $\mathcal{T}$ that breaks the $\ell$-DHE assumption with non-negligible probability $\nu$. First, $\mathcal{T}$ receives an instance $\left(e, \mathbb{G}, \tilde{\mathbb{G}}, \mathbb{G}_{t}, p, g, \tilde{g}, g_{1}, \tilde{g}_{1}\right.$, $\left.\ldots, g_{\ell}, \tilde{g}_{\ell}, g_{\ell+2}, \ldots, g_{2 \ell}\right)$ of the $\ell$-DHE assumption. $\mathcal{T}$ sets $p a r \leftarrow\left(p, \mathbb{G}, \tilde{\mathbb{G}}, \mathbb{G}_{t}, e, g, \tilde{g}, g_{1}, \tilde{g}_{1}, \ldots, g_{\ell}, \tilde{g}_{\ell}, g_{\ell+2}, \ldots, g_{2 \ell}\right)$ and sends par to $\mathcal{A}$. $\mathcal{A}$ returns $\left(v c, i, x, x^{\prime}, w, w^{\prime}\right)$ such that VC.Verify $(p a r, v c, x, i, w)=1$, VC.Verify $\left(\right.$ par $\left., v c, x^{\prime}, i, w^{\prime}\right)$ $=1, i \in[1, \ell], x, x^{\prime} \in \mathcal{M}$, and $x \neq x^{\prime} . \mathcal{T}$ computes $g_{\ell+1}$ as follows:

$$
\begin{aligned}
e(w, \tilde{g}) e\left(g_{1}, \tilde{g}_{\ell}\right)^{x} & =e\left(w^{\prime}, \tilde{g}\right) e\left(g_{1}, \tilde{g}_{\ell}\right)^{x^{\prime}} \Rightarrow \\
e\left(w / w^{\prime}, \tilde{g}\right) & =e\left(g_{1}, \tilde{g}_{\ell}\right)^{x^{\prime}-x} \Rightarrow \\
e\left(\left(w / w^{\prime}\right)^{1 /\left(x^{\prime}-x\right)}, \tilde{g}\right) & =e\left(g_{1}, \tilde{g}_{\ell}\right) \Rightarrow \\
e\left(\left(w / w^{\prime}\right)^{1 /\left(x^{\prime}-x\right)}, \tilde{g}\right) & =e\left(g_{\ell+1}, \tilde{g}\right) .
\end{aligned}
$$

The last equation implies that $g_{\ell+1}=\left(w / w^{\prime}\right)^{1 /\left(x^{\prime}-x\right)} \cdot \mathcal{T}$ returns $\left(w / w^{\prime}\right)^{1 /\left(x^{\prime}-x\right)}$ as a solution for the $\ell$-DHE problem.

\section{APPENDIX K \\ ApPLiCATION OF FUnCTIONALITY $\mathcal{F}_{\text {CD }}$ TO EFFICIENT OR PROOFS}

Intuition. Despite a large number of use cases for OR proofs, they are usually avoided due to their inefficiency: typically the size of a zero-knowledge proof for an OR-relation grows with the number $N$ of values involved. We illustrate how $\mathcal{F}_{\mathrm{CD}}$ and $\mathcal{F}_{\text {NIC }}$ can be used to compute efficient zero-knowledge proofs for OR-relations. In a nutshell, the protocol works as follows. Let $\mathrm{x}$ be a list of $N$ values. The prover wishes to prove that at least one of the values fulfils a statement. First, the prover writes $\mathbf{x}$ into the committed database by using the cd.write interface of $\mathcal{F}_{\mathrm{CD}}$. Let $\mathbf{x}[i](i \in[1, N])$ be a value that fulfils the statement. Then, the prover obtains commitments com $_{i}$ and com $_{r}$ from $\mathcal{F}_{\text {NIC }}$ to the position $i$ and the value $v=\mathbf{x}[i]$, respectively. The prover uses the cd.read interface of functionality $\mathcal{F}_{\mathrm{CD}}$ to prove that $\operatorname{com}_{i}$ and $\operatorname{com}_{r}$ together commit to an entry $[i, v]$ that is stored in the database. Finally, the prover uses functionality $\mathcal{F}_{\mathrm{ZK}}^{R}$ for the relation $R$ defined by the required statement to prove that the value $v$ in $\mathrm{com}_{r}$ fulfils the statement. In our protocol, the cost of writing the list of $N$ values into the database grows with $N$. However, after that, the prover can compute multiple OR proofs of size independent of $N$, and thus the cost of writing the values into the database is amortized. We remark that our protocol is also applicable when the OR proof involves a subset of the values. For example, if only the values $\mathbf{x}[i]$ for $i \in\left[1, N^{\prime}\right]$, with $N^{\prime}<N$, are involved, the prover uses a range proof to prove that $i \in\left[1, N^{\prime}\right]$, where $i$ is the position in the commitment $\mathrm{com}_{i}$ read from the database. OR proof example. Let KeyGen, Sign and VfSig be a signature scheme and let CSetup, Com and VfCom be a commitment scheme as defined in Section VI-A. Consider a protocol where the prover must prove to the verifier, at separate steps in the protocol, three statements: (1) that he possesses a signature $s$ verifiable with public key $p k$ on $N$ messages $\left(m_{1}, \ldots, m_{N}\right)$, (2) that a signed message $m_{i}(i \in[1, N])$ fulfills $m_{i} \geq 18$ and (3) that, without revealing $i$, at least one of the signed messages $m_{i}$ fulfils $m_{i} \geq 65$.

For the sake of comparison, in Appendix K-A, for this example we describe a protocol that does not use any mechanism to store information between prover and verifier, and a protocol that uses a commitment scheme. In Appendix K-B, we describe a protocol that uses $\mathcal{F}_{\mathrm{CD}}$. The basic idea is that the protocol that does not store information between prover and verifier needs to repeat the proof of signature possession computed for statement (1) again for statements (2) and (3). The protocol that uses commitments does not need to repeat the proof of signature possession because it stores the messages signed into commitments, but requires an inefficient OR proof for statement (3). Finally the protocol that uses $\mathcal{F}_{\mathrm{CD}}$ does not repeat the proof of signature possession and provides an efficient OR proof for statement (3).

\section{A. Protocols For OR Proofs without $\mathcal{F}_{\mathrm{CD}}$}

We compare our protocol to protocols that do not use $\mathcal{F}_{\mathrm{CD}}$. First, we describe a protocol that does not use commitments. Second, we show a protocol that uses a commitment scheme CSetup, Com and VfCom.

Protocol that does not use commitments. The prover and the verifier use the functionalities $\mathcal{F}_{\mathrm{ZK}}^{R_{1}}, \mathcal{F}_{\mathrm{ZK}}^{R_{2 i}}(i \in[1, N])$ and $\mathcal{F}_{\mathrm{ZK}}^{R_{3}}$, where the relations $R_{1}, R_{2 i}$ and $R_{3}$ are defined as follows.

$$
R_{1}=\left\{\left(\text { wit }_{1}, \text { ins }_{1}\right): 1=\operatorname{VfSig}\left(p k, s,\left\langle m_{1}, \ldots, m_{N}\right\rangle\right)\right\}
$$

with wit $_{1}=\left(s, m_{1}, \ldots, m_{N}\right)$ and $i n s_{1}=p k$,

$$
\begin{aligned}
R_{2 i}= & \left\{\left(w_{i t} t_{2 i}, \text { ins }_{2 i}\right): 1=\operatorname{VfSig}\left(p k, s,\left\langle m_{1}, \ldots, m_{N}\right\rangle\right) \wedge\right. \\
& \left.m_{i} \geq 18\right\}
\end{aligned}
$$


with wit $_{2 i}=\left(s, m_{1}, \ldots, m_{N}\right)$ and $i n s_{2 i}=p k$ (here we abstract away how $m_{i} \geq 18$ is proven),

$$
\begin{aligned}
& R_{3}=\left\{\left(\text { wit }_{3}, \text { ins }_{3}\right): 1=\operatorname{VfSig}\left(p k, s,\left\langle m_{1}, \ldots, m_{N}\right\rangle\right) \wedge\right. \\
& \left.\left(m_{1} \geq 65 \vee \ldots \vee m_{N} \geq 65\right)\right\}
\end{aligned}
$$

with wit $_{3}=\left(s, m_{1}, \ldots, m_{N}\right)$ and $i n s_{3}=p k$ (here we abstract away how $m_{i} \geq 65$ is proven).

In this case, because commitments are not used, relation $R_{2 i}$ and relation $R_{3}$ need to repeat the proof of signature possession.

Protocol that uses commitments. By using the commitment scheme (CSetup, Com, VfCom), the relations $R_{1}, R_{2 i}$ and $R_{3}$ are defined as follows.

$$
\begin{aligned}
R_{1}= & \left\{\left(\text { wit }_{1}, \text { ins }_{1}\right): 1=\operatorname{VfSig}\left(p k, s,\left\langle m_{1}, \ldots, m_{N}\right\rangle\right) \wedge\right. \\
& 1=\operatorname{VfCom}\left(\text { par }_{c}, \text { com }_{1}, m_{1}, \text { open }_{1}\right) \wedge \ldots \wedge \\
& \left.1=\operatorname{VfCom}\left(\text { par }_{c}, \text { com }_{N}, m_{N}, \text { open }_{N}\right)\right\}
\end{aligned}
$$

with witness wit $_{1}=\left(s, m_{1}\right.$, open $_{1}, \ldots, m_{N}$, open $\left._{N}\right)$ and instance $i n s_{1}=\left(p k, \operatorname{par}_{c}, \operatorname{com}_{1}, \ldots, \operatorname{com}_{N}\right)$,

$$
\begin{aligned}
R_{2 i}= & \left\{\left(\text { wit }_{2 i}, \text { ins }_{2 i}\right): 1=\mathrm{VfCom}\left(\text { par }_{c}, \text { com }_{i}, m_{i}, \text { open }_{i}\right) \wedge\right. \\
& \left.m_{i} \geq 18\right\}
\end{aligned}
$$

with wit $_{2 i}=\left(m_{i}\right.$, open $\left._{i}\right)$ and ins $_{2 i}=\left(\right.$ par $_{c}$, com $\left._{i}\right)$ (here we abstract away how $m_{i} \geq 18$ is proven),

$$
\begin{aligned}
R_{3}= & \left\{\left(\text { wit }_{3}, \text { ins }_{3}\right):\right. \\
& \left(1=\operatorname{VfCom}\left(\text { par }_{c}, \text { com }_{1}, m_{1}, \text { open }_{1}\right) \wedge m_{1} \geq 65\right) \\
& \vee \ldots \vee \\
& \left.\left(1=\operatorname{VfCom}\left(\text { par }_{c}, \text { com }_{N}, m_{N}, \text { open }_{N}\right) \wedge m_{N} \geq 65\right)\right\}
\end{aligned}
$$

with wit $_{3}=\left(m_{1}\right.$, open $_{1}, \ldots, m_{N}$, open $\left._{N}\right)$ and ins $_{3}=$ $\left(\operatorname{par}_{c}, \operatorname{com}_{1}, \ldots, \operatorname{com}_{N}\right)$ (here we abstract away how $m_{i} \geq 65$ is proven).

The prover computes the commitments $\left(\operatorname{com}_{1}, \ldots, \mathrm{com}_{N}\right)$. As can be seen, now the size of wit $_{2 i}$ does not grow with $N$, and thus constructions for $\mathcal{F}_{\mathrm{ZK}}^{R_{2 i}}(i \in[1, N])$ can be more efficient. However, the cost of the proof for statement (3) grows with $N$.

\section{B. Protocol For OR Proofs with $\mathcal{F}_{\mathrm{CD}}$}

The prover and the verifier use the functionalities $\mathcal{F}_{\mathrm{ZK}}^{R_{1}}, \mathcal{F}_{\mathrm{ZK}}^{R_{2 i}}$ ( $i \in[1, N]$ ) and $\mathcal{F}_{\mathrm{ZK}}^{R_{3}}$, where the relations $R_{1}, R_{2 i}$ and $R_{3}$ are defined as follows.

$$
\begin{aligned}
& R_{1}=\left\{\left(\text { wit }_{1}, \text { ins }_{1}\right):\right. \\
& 1=\operatorname{VfSig}\left(p k, s,\left\langle m_{1}, \ldots, m_{N}\right\rangle\right) \wedge \\
& 1=\text { COM.Verify }\left(\text { parcom }_{\text {com }}, m_{1} \text {, open } \text { op }_{1}\right) \wedge \\
& \text {... } \wedge \\
& \left.1=\text { COM.Verify }\left(\text { parcom }_{\text {com }}, m_{N}, \text { open }_{N}\right)\right\} \text {, }
\end{aligned}
$$

with wit $_{1}=\left(s, m_{1}\right.$, open $_{1}, \ldots, m_{N}$, open $\left._{N}\right)$ and ins $_{1}=(p k$, $\left.\operatorname{parcom} \operatorname{com}_{1}, \ldots, \operatorname{com}_{N}\right)$,

$$
\begin{aligned}
R_{2 i}= & \left\{\left(\text { wit }_{2 i}, \text { ins }_{2 i}\right):\right. \\
& 1=\text { COM.Verify }\left(\text { parcom }, \text { com }_{i}, m_{i}, \text { open }_{i}\right) \wedge \\
& \left.m_{i} \geq 18\right\},
\end{aligned}
$$

with wit $_{2 i}=\left(m_{i}\right.$, open $\left._{i}\right)$ and ins $_{2 i}=\left(\right.$ parcom, com $\left._{i}\right)$ (here we abstract away how $m_{i} \geq 18$ is proven),

$$
\begin{aligned}
R_{3}= & \left\{\left(\text { wit }_{3}, \text { ins }_{3}\right):\right. \\
& 1=\text { COM.Verify }(\text { parcom }, \text { com }, m, \text { open }) \wedge \\
& m \geq 65\},
\end{aligned}
$$

with wit $_{3}=(m$,open $)$ and ins $_{3}=($ parcom, com $)$ (here we abstract away how $m_{i} \geq 65$ is proven). In relation $R_{3}$, com is a commitment to a message $m \in\left(m_{1}, \ldots, m_{N}\right)$ that fulfills the statement $m \geq 65$. The prover uses $\mathcal{F}_{\mathrm{CD}}$ to prove that the message $m$ committed in com belongs to $\left(m_{1}, \ldots, m_{N}\right)$.

Note that the size of neither wit $_{2 i}$ nor wit $_{3}$ grows with $N$. In the case of $R_{3}$, this is due to the use of $\mathcal{F}_{\mathrm{CD}}$ to read one message such that $m \geq 65$. We recall that, in our construction for $\mathcal{F}_{\mathrm{CD}}$ in Appendix $\mathrm{H}$, the communication cost of a reading operation is independent of the size of the table. We also note that, if the OR proof must be done over a subset of the data in the table, e.g. $\left[1, N^{\prime}\right]$ for $N^{\prime}<N$, the size of wit $_{3}$ does not grow with $N^{\prime}$ either. In such a case, we would modify $R_{3}$ as follows:

$$
\begin{aligned}
R_{3}= & \left\{\left(\text { wit }_{3}, \text { ins }_{3}\right):\right. \\
& 1=\text { COM.Verify }(\text { parcom }, \text { com }, \text { m, open }) \wedge \\
& m \geq 65 \wedge \\
& 1=\text { COM.Verify }\left(\text { parcom }, \text { com }^{\prime}, i, \text { open }^{\prime}\right) \wedge \\
& \left.i \in\left[1, N^{\prime}\right]\right\} .
\end{aligned}
$$

Here $\mathrm{com}^{\prime}$ is a commitment to the position $i$ where the message $m$ is stored in the array kept by $\mathcal{F}_{\mathrm{CD}}$.

We describe how the protocols works. First, the prover $\mathcal{P}$ sets up $\mathcal{F}_{\text {NIC }}$ and obtains commitments $\left(\operatorname{com}_{1}, \ldots, \operatorname{com}_{N}\right)$ to the messages $\left(m_{1}, \ldots, m_{N}\right)$ from $\mathcal{F}_{\text {NIC }}$ (see Figure 13).

To prove the first statement, the prover uses the functionality $\mathcal{F}_{\mathrm{ZK}}^{R_{1}}$. The verifier $\mathcal{V}$ uses $\mathcal{F}_{\text {NIC }}$ to validate the commitments in the instance (see Figure 14).

After that, the prover writes the messages $\left(m_{1}, \ldots, m_{N}\right)$ into the table $\mathrm{Tbl}_{c d}$ of $\mathcal{F}_{\mathrm{CD}}$. For this purpose, the prover obtains from $\mathcal{F}_{\text {NIC }}$ commitments to the positions $[1, N] . \mathcal{F}_{\mathrm{CD}}$ is parameterized by $N_{\max }=N$ and $U_{v}$, which is set to the message space of the signature scheme (see Figure 15).

The prover must then disclose the opening of the commitments to the positions $\left(\operatorname{com}_{1}^{\prime}, \ldots, \operatorname{com}_{N}^{\prime}\right)$ to the verifier, e.g. by using functionality $\mathcal{F}_{\mathrm{SMT}}$ in Appendix F. After that, the verifier verifies the openings by using $\mathcal{F}_{\mathrm{NIC}}$ (see Figure 16).

To prove the second and the third statements, the prover uses the functionalities $\mathcal{F}_{\mathrm{ZK}}^{R_{2 i}}(i \in[1, N])$ and $\mathcal{F}_{\mathrm{ZK}}^{R_{3}}$. For the case of $R_{3}$, the prover chooses an index $i \in[1, N]$ such that $m_{i} \geq 65$. The prover then obtains a commitment $\mathrm{com}^{\prime}$ to $i$ and

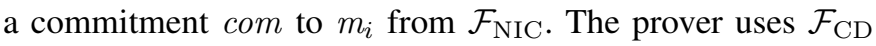
to prove that there is an entry $\left[i, m_{i}\right]$ stored in state. Finally, the prover sets wit $_{3}=\left(m_{i}\right.$,open $)$ and ins $_{3}=($ parcom, com $)$ and uses $\mathcal{F}_{\mathrm{ZK}}^{R_{3}}$ to prove that $m_{i} \geq 65$ (see Figure 17). 


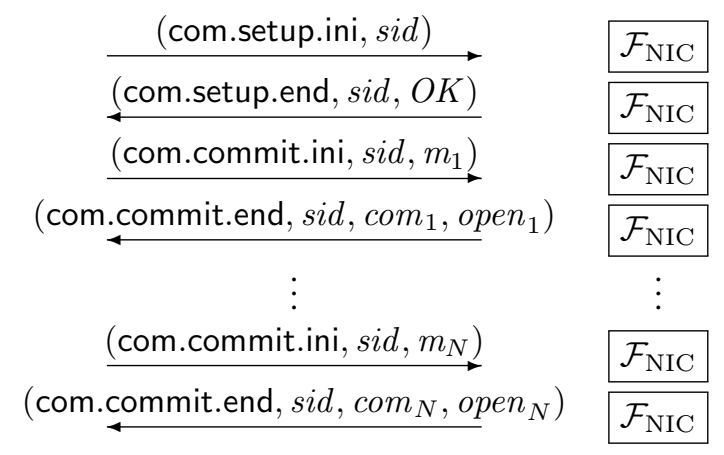

Fig. 13. Protocol: phase 1

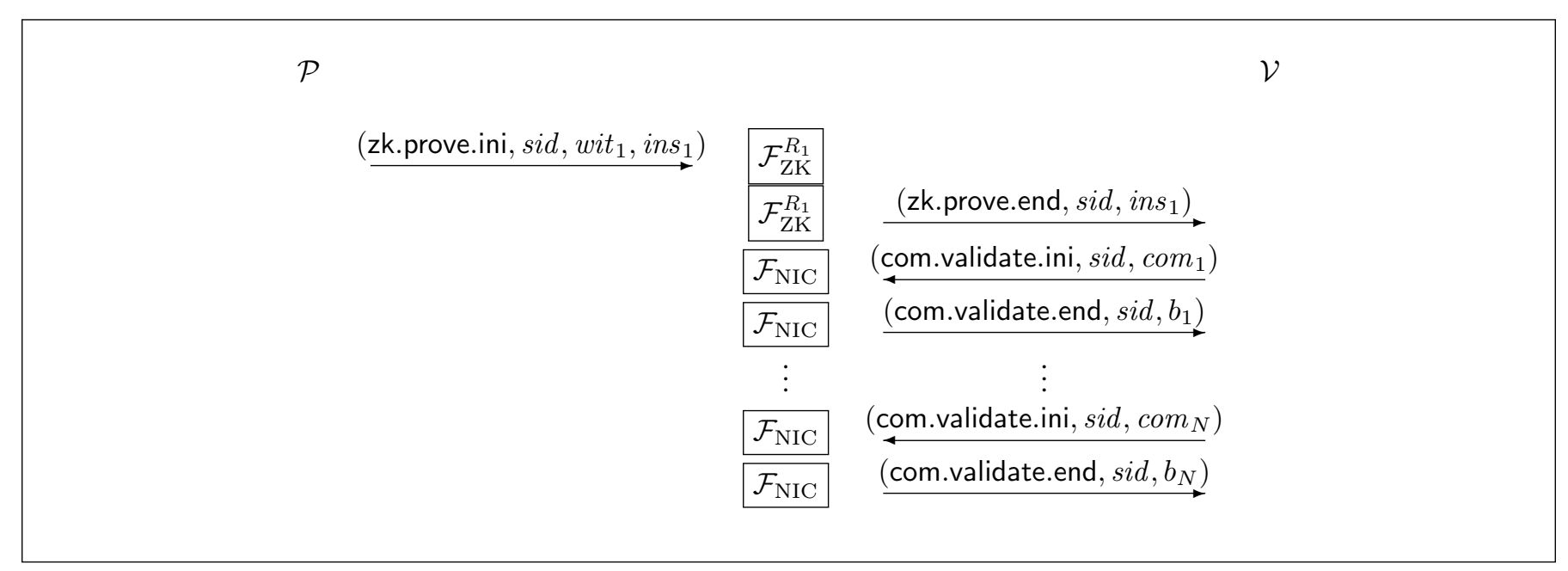

Fig. 14. Protocol: phase 2

\section{APPENDIX L \\ IdEAL Functionality $\mathcal{F}_{\text {ZKC }}^{R}$ FOR ZERo-KNOWLEdGE COUNTING}

Let $R$ be a polynomial time computable binary relation. For tuples (wit, ins $) \in R$ we call wit the witness and ins the instance. Let $L$ be the NP-language consisting of the instances ins for which there exist witnesses wit such that (wit, ins $) \in$ $R$.

We consider relations $R$ such that, for all instances ins $\in L$, the valid witnesses $\left(\right.$ wit $_{1}, \ldots$, wit $\left._{N}\right)$ belong to a finite list $S$ of size $N$. We define zero-knowledge counting as the task of both proving knowledge of a witness wit such that (wit, ins) $\in R$ (as in the functionality for zero-knowledge described in Appendix D), and of counting the number of times each of the witnesses $\left(\right.$ wit $_{1}, \ldots$, wit $\left._{N}\right)$ was used. For simplicity, our functionality $\mathcal{F}_{\mathrm{ZKC}}^{R}$ considers relations where each witness is treated as a single element. If a witness consists of a tuple of elements, and the values of those elements belong to a finite list, it is possible to define and realize a more involved functionality that stores one counter for each of the elements in the witness.

Our functionality $\mathcal{F}_{\mathrm{ZKC}}^{R}$ in Figure 18 interacts with a prover $\mathcal{P}$ and a verifier $\mathcal{V} . \mathcal{F}_{\mathrm{ZKC}}^{R}$ is parameterized by a relation $R$, which describes the list of possible witnesses $\left(\right.$ wit $_{1}, \ldots$, wit $\left._{N}\right)$. $\mathcal{F}_{\mathrm{ZKC}}^{R}$ maintains a table Tbl. Tbl contains $N$ entries of the form $\left[\right.$ wit $\left._{i}, c t_{i}\right](i \in[1, N])$, where wit $_{i} \in S$ is a possible witness value and $c t_{i}$ is a counter that stores the number of times that the prover used the witness value wit $_{i}$.

The prover $\mathcal{P}$ proves to the verifier $\mathcal{V}$ knowledge of a witness value wit $_{i}$ such that, for an instance ins, $\left(\right.$ wit $_{i}$, ins $) \in R$. The prover $\mathcal{P}$ can also reveal to the verifier $\mathcal{V}$ the number $c t_{i}$ of times that a witness value wit $_{i}$ was used. We note that it is possible to define more involved functionalities where the prover $\mathcal{P}$, instead of revealing $c t_{i}$, proves e.g. that $c t_{i}$ belongs to an interval, or, in general, proves in zero-knowledge any statistic about the counters stored in Tbl. 


$$
\begin{aligned}
& \text { (com.commit.ini, sid, 1) } \\
& \text { (com.commit.end, sid, } \text { com }_{1}^{\prime}, \text { open }_{1}^{\prime} \text { ) } \\
& \vdots \\
& \text { (com.commit.ini, sid, } N \text { ) } \\
& \text { (com.commit.end, sid, } \operatorname{com}_{N}^{\prime}, \text { open }_{N}^{\prime} \text { ) } \\
& \text { (cd.write.ini, sid, } \text { com }_{1}^{\prime}, 1, \text { open }_{1}^{\prime}, \text { com }_{1}, m_{1}, \text { open }_{1} \text { ) } \\
& \vdots \\
& \text { (cd.write.ini, } \left.\operatorname{sid,\operatorname {com}_{N}^{\prime },N\text {,open}}{ }_{N}, \operatorname{com}_{N}, m_{N}, \text { open }_{N}\right)
\end{aligned}
$$

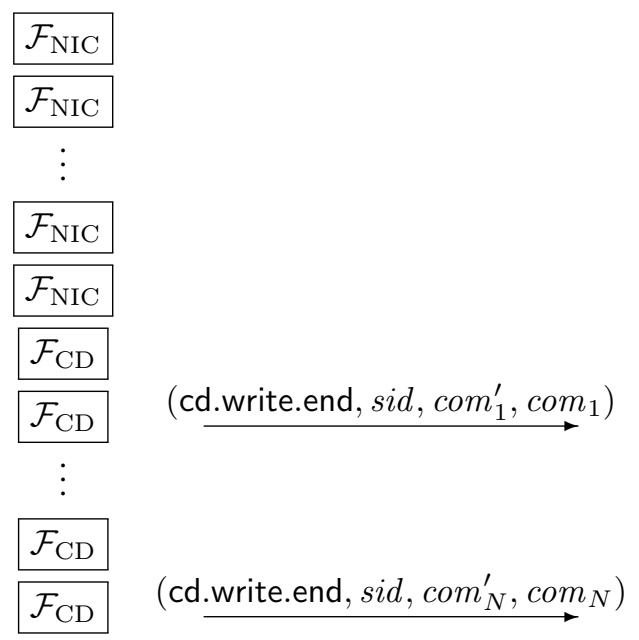

Fig. 15. Protocol: phase 3

$\mathcal{P}$

$$
\begin{aligned}
& \text { (smt.send.ini, sid, } \left.\left\langle\text { open }_{1}^{\prime}, \ldots, \text { open }_{N}^{\prime}\right\rangle\right) \quad \mathcal{F}_{\mathrm{SMT}} \\
& \left.\mathcal{F}_{\mathrm{SMT}} \quad \text { (smt.send.end, sid, }\left\langle\mathrm{open}_{1}^{\prime}, \ldots, \text { open }_{N}^{\prime}\right\rangle\right) \\
& \mathcal{F}_{\mathrm{NIC}} \quad \text { (com.verify.ini, sid, } \text { com }_{1}^{\prime}, 1, \text { open }_{1}^{\prime} \text { ) } \\
& \begin{array}{c}
\mathcal{F}_{\text {NIC }} \\
\vdots
\end{array}
\end{aligned}
$$

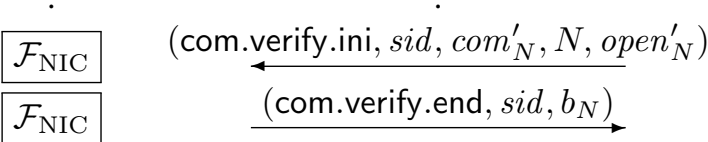

Fig. 16. Protocol: phase 4

The interaction between the functionality $\mathcal{F}_{\mathrm{ZKC}}^{R}$, the prover $\mathcal{P}$ and the verifier $\mathcal{V}$ takes place through the following interfaces.

1) The verifier $\mathcal{V}$ uses the zkc.setup interface to initialize to 0 the counters in the table Tbl.

2) The prover $\mathcal{P}$ uses the zkc.prove interface to prove to the verifier $\mathcal{V}$ knowledge of a witness value wit $_{i}$ such that, for an instance ins, $\left(\right.$ wit $_{i}$, ins $) \in R$. This interface is similar to the zk.prove interface of the functionality $\mathcal{F}_{\mathrm{ZK}}^{R}$ described in Appendix D. The main difference is that now the $\mathcal{F}_{\mathrm{ZKC}}^{R}$ updates a counter of the number of times that the witness wit $_{i}$ is used.

3) The prover $\mathcal{P}$ uses the zkc.count interface to reveal to the verifier $\mathcal{V}$ that the witness value wit $_{i}$ was used $c t_{i}$ times.

\section{APPENDIX M \\ Construction $\Pi_{\text {ZKC }}^{R}$ FOR ZERo-Knowledge Counting}

We propose a construction $\Pi_{\mathrm{ZKC}}^{R}$ that realizes the ideal functionality $\mathcal{F}_{\mathrm{ZKC}}^{R}$ described in Appendix L. We describe our construction $\Pi_{\mathrm{ZKC}}^{R}$ as a hybrid protocol that uses several ideal functionalities as building blocks. Thanks to that, the security analysis is simpler and the construction allows for multiple instantiations obtained via replacing the ideal functionalities by protocols that realized them.

Our construction uses the functionality $\mathcal{F}_{\mathrm{CD}}$ for a committed database described in Section III, which is used to store the number of times each of the witnesses $\left(\right.$ wit $_{1}, \ldots$, wit $\left._{N}\right)$ is used. It also uses the functionality $\mathcal{F}_{\mathrm{NIC}}$ for non-interactive commit- 


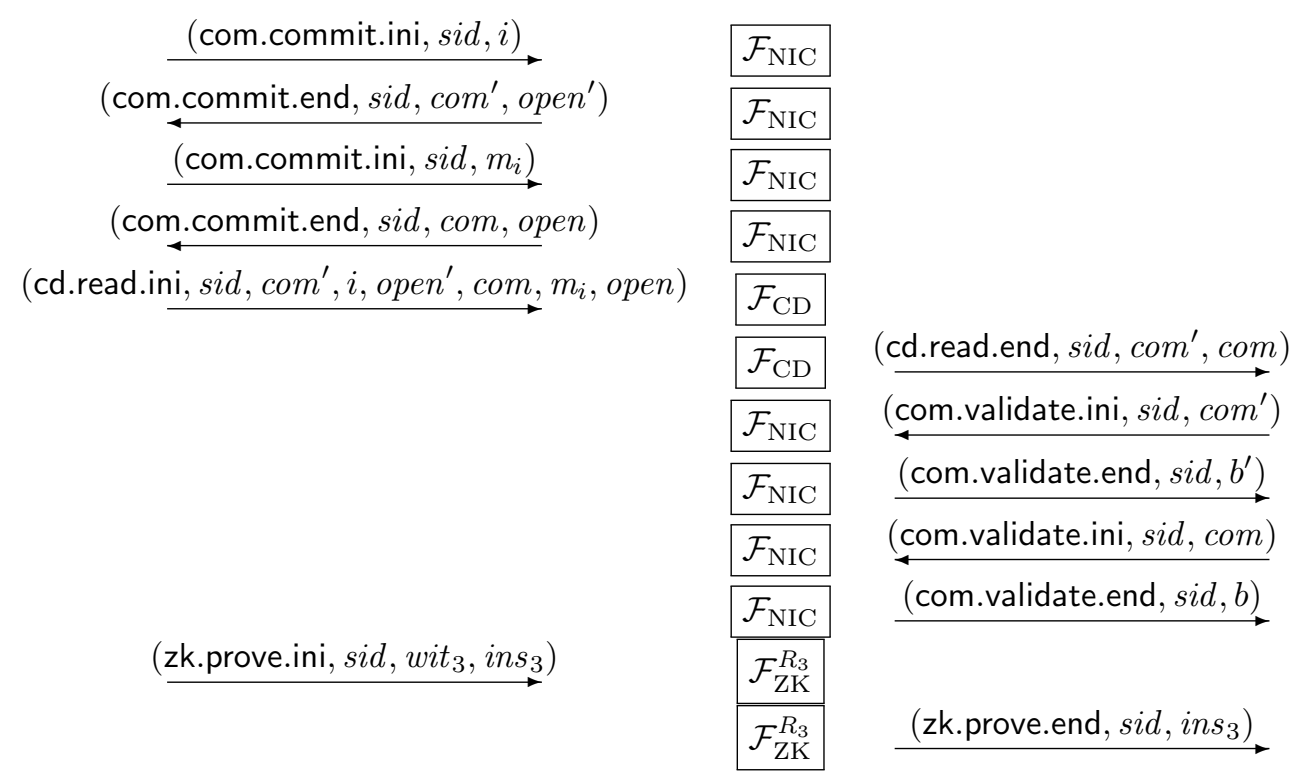

Fig. 17. Protocol: phase 5

ments described in Appendix $\mathrm{E}$ and the functionality $\mathcal{F}_{\mathrm{SMT}}$ for secure message transmission described in Appendix F. Additionally, it uses the functionality $\mathcal{F}_{\mathrm{ZK}}^{R_{c}}$ for zero-knowledge described in Appendix D.

Let $f: S \rightarrow[1, N]$ be a bijective function that maps each possible witness $\left(\right.$ wit $_{1}, \ldots$, wit $\left._{N}\right)$ to an array position. The relation $R_{c}$, which extends the relation $R$, is defined as follows:

$$
\begin{aligned}
& R_{c}=\left\{\left(\text { wit }_{c}, \text { ins }_{c}\right):\right. \\
& \left(\text { wit }_{i}, \text { ins }\right) \in R \wedge \\
& i=f\left(\text { wit }_{i}\right) \wedge \\
& 1=\text { COM.Verify }(\text { parcom }, \text { com }, i, \text { open }) \wedge
\end{aligned}
$$

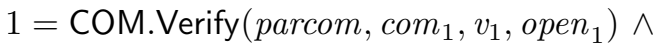

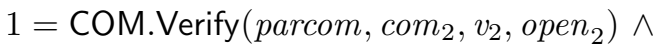

$$
\begin{aligned}
& \left.v_{2}=v_{1}+1 \wedge v_{2} \in\left[0, c t_{\text {max }}\right]\right\}
\end{aligned}
$$

where the witness wit $_{c}$ is $\left(\right.$ wit $_{i}, i$, open, $v_{1}$, open $_{1}, v_{2}$, open $\left._{2}\right)$ and the instance ins $_{c}$ is (ins, parcom, com, com 1, com $_{2}, c p$ ). Equation 26 corresponds to the relation $R$. Equation 27 maps the witness value wit $_{i}$ to a value $i \in[1, N]$. In equation 28 , equation 29 and equation 30, the prover proves that com, com 1 and $\mathrm{com}_{2}$ are commitments to $i, v_{1}$ and $v_{2}$ respectively. In equation 31, the prover proves that $v_{2}$ increments $v_{1}$.

By using $\mathcal{F}_{\mathrm{ZK}}^{R_{\mathrm{C}}}$, the prover proves to the verifier that he knows a witness wit $_{i}$ such that $\left(\right.$ wit $_{i}$, ins $) \in R$. Additionally, the prover computes commitments to the position $i$ and to two values $v_{1}$ and $v_{2}$ such that $v_{2}=v_{1}+1$ and proves that the function $f$ associates the position $i$ with the witness $w_{i t}$.
These commitments will be sent as input to the functionality $\mathcal{F}_{\mathrm{CD}}$. The position $i$ indicates the position in the array of $\mathcal{F}_{\mathrm{CD}}$, the value $v_{1}$ is the old value of the counter of the number of times wit $_{i}$ was used by the prover, and the value $v_{2}$ is the new value of that counter.

We describe the construction $\Pi_{\mathrm{ZKC}}^{R}$ in Figure 19, Figure 20 and Figure 21. In Figure 22 and Figure 23, we show the message flow for the zkc. prove and zkc.count interfaces respectively. We recall that the functionality $\mathcal{F}_{\mathrm{ZKC}}^{R}$ is parameterized by a description of a relation $R$, which describes the list of possible witnesses $\left(\right.$ wit $_{1}, \ldots$, wit $\left._{N}\right)$, and by an upper bound $c t_{\max }$ on the number of times a witness value wit $_{i}(i \in[1, N])$ can be used.

\section{APPENDIX N \\ SECURity ANALYSis OF CONSTRUCTION $\Pi_{\mathrm{ZKC}}^{R}$}

Theorem N.1: The construction $\Pi_{\mathrm{ZKC}}^{R}$ securely realizes $\mathcal{F}_{\mathrm{ZKC}}^{R}$ in the $\mathcal{F}_{\mathrm{CD}}, \mathcal{F}_{\mathrm{ZK}}^{R_{c}}, \mathcal{F}_{\mathrm{SMT}}$, and $\mathcal{F}_{\mathrm{NIC}}$-hybrid model.

To prove that our construction $\Pi_{\mathrm{ZKC}}^{R}$ securely realizes the ideal functionality $\mathcal{F}_{\mathrm{ZKC}}^{R}$, we have to show that for any environment $\mathcal{Z}$ and any adversary $\mathcal{A}$ there exists a simulator $\mathcal{S}$ such that $\mathcal{Z}$ cannot distinguish whether it is interacting with $\mathcal{A}$ and the protocol in the real world or with $\mathcal{S}$ and $\mathcal{F}_{\mathrm{ZKC}}^{R}$. The simulator thereby plays the role of all honest parties in the real world and interacts with $\mathcal{F}_{\mathrm{ZKC}}^{R}$ for all corrupt parties in the ideal world.

Our simulator $\mathcal{S}$ runs copies of the functionalities $\mathcal{F}_{\mathrm{CD}}$, $\mathcal{F}_{\mathrm{ZK}}^{R_{c}}, \mathcal{F}_{\mathrm{SMT}}$ and $\mathcal{F}_{\mathrm{NIC}}$. When any of the copies of those functionalities aborts, $\mathcal{S}$ implicitly forwards the abortion 
$\mathcal{F}_{\mathrm{ZKC}}^{R}$ is parameterized by a description of a relation $R$, which describes the list of possible witnesses $\left(\right.$ wit $_{1}, \ldots$, wit $\left._{N}\right)$, and by an upper bound $c t_{\max }$ on the number of times a witness value wit $i_{i}(i \in[1, N])$ can be used. $\mathcal{F}_{\mathrm{ZKC}}^{R}$ interacts with a prover $\mathcal{P}$ and a verifier $\mathcal{V}$.

1) On input (zkc.setup.ini, sid) from $\mathcal{V}$ :

- Abort if $\operatorname{sid} \notin\left(\mathcal{P}, \mathcal{V}, \operatorname{sid}^{\prime}\right)$ or if $(s i d, c v)$ is already stored.

- Initialize a counter $c v \leftarrow 0$ for the verifier and store $(s i d, c v)$.

- Send (zkc.setup.sim, sid) to $\mathcal{S}$.

S. On input (zkc.setup.rep, sid) from $\mathcal{S}$ :

- Abort if $(s i d, c v)$ is not stored, or if $(s i d$, Tbl, $c p)$ is already stored.

- Initialize an empty table Tbl with entries $\left[w_{i t}, 0\right]$ for $i=1$ to $N$.

- Initialize a counter $c p \leftarrow 0$ for the prover and store $(s i d, \mathrm{Tbl}, c p)$.

- Send (zkc.setup.end, sid) to $\mathcal{P}$.

2) On input (zkc.prove.ini, sid, wit ${ }_{i}$, ins) from $\mathcal{P}$ :

- Abort if $($ sid, Tbl, $c p)$ is not stored or if $\left(w_{i} t_{i}\right.$, ins $) \notin R$.

- Retrieve the entry $\left[w i t_{i}, c t_{i}\right]$ in Tbl. Set $c t_{i}^{\prime} \leftarrow c t_{i}+1$. If $c t_{i}^{\prime}>c t_{\max }$, abort, else store $\left[w i t_{i}, c t_{i}^{\prime}\right]$ in Tbl.

- Increment the counter $c p$ in $(s i d, \mathrm{Tbl}, c p)$.

- Create a fresh qid and store (qid, ins, $c p$ ).

- Send (zkc.prove.sim, sid, qid, ins) to $\mathcal{S}$.

S. On input (zkc.prove.rep, sid, qid) from $\mathcal{S}$ :

- Abort if (qid, ins, $\left.c p^{\prime}\right)$ is not stored, or if $c p^{\prime} \neq c v+1$, where $c v$ is stored in (sid, $\left.c v\right)$.

- Increment the counter $c v$ in $(s i d, c v)$.

- Delete the record (qid, ins, $\left.c p^{\prime}\right)$.

- Send (zkc.prove.end, sid, ins) to $\mathcal{V}$.

3) On input (zkc.count.ini, sid, wit ${ }_{i}$ ) from $\mathcal{P}$ :

- Abort if (sid, Tbl, $c p)$ is not stored or if wit $_{i} \notin\left(\right.$ wit $_{1}, \ldots$, wit $\left._{N}\right)$.

- Retrieve the entry $\left[w_{i} t_{i}, c t_{i}\right]$ in Tbl, create a fresh qid and store $\left(q i d, w_{i} t_{i}, c t_{i}, c p\right)$.

- Send (zkc.count.sim, sid, qid) to $\mathcal{S}$.

S. On input (zkc.count.rep, sid, qid) from $\mathcal{S}$ :

- Abort if $\left(q i d, w_{i t}, c t_{i}^{\prime}, c p^{\prime}\right)$ is not stored or if $c p^{\prime} \neq c v$, where $c v$ is stored in $(s i d, c v)$.

- Delete the record $\left(q i d, w_{i} t_{i}, c t_{i}^{\prime}, c p^{\prime}\right)$.

- Send (zkc.count.end, sid, wit $\left.t_{i}, c t_{i}^{\prime}\right)$ to $\mathcal{V}$.

Fig. 18. Functionality $\mathcal{F}_{\mathrm{ZKC}}^{R}$

$\Pi_{\mathrm{ZKC}}^{R}$ uses $\mathcal{F}_{\mathrm{CD}}$, which is parameterized by a universe of state values $U_{v}=\left[0, c t_{\text {max }}\right]$ and by a maximum state size

$N_{\max }=N$. It also uses the functionality $\mathcal{F}_{\text {NIC }}$ for non-interactive commitments, the functionality $\mathcal{F}_{\mathrm{ZK}}^{R_{c}}$ for zero-knowledge and the functionality $\mathcal{F}_{\mathrm{SMT}}$ for secure message transmission.

1) On input (zkc.setup.ini, sid), $\mathcal{V}$ and $\mathcal{P}$ do the following:

- $\mathcal{V}$ aborts if $\operatorname{sid} \notin(\mathcal{P}, \mathcal{V}$, sid $)$ or if $(s i d, c v)$ is already stored.

- $\mathcal{V}$ initializes a counter $c v \leftarrow 0$ and stores $(s i d, c v)$.

- $\mathcal{V}$ sends (com.setup.ini, sid) to $\mathcal{F}_{\mathrm{NIC}}$ and receives (com.setup.end, sid, $O K$ ) from $\mathcal{F}_{\mathrm{NIC}}$.

- $\mathcal{V}$ initializes a table Tbl with entries $\left[w_{i} t_{i}, 0\right]$ for $i=1$ to $N$, and sends (cd.setup.ini, sid, Tbl) to $\mathcal{F}_{\mathrm{CD}}$.

- $\mathcal{P}$ receives (cd.setup.end, sid, $\mathrm{Tbl}$ ) from $\mathcal{F}_{\mathrm{CD}}$.

- $\mathcal{P}$ aborts if Tbl does not consist of entries $\left[\right.$ wit $\left._{i}, 0\right]$ for $i=1$ to $N$.

- $\mathcal{P}$ sends (com.setup.ini, sid) to $\mathcal{F}_{\mathrm{NIC}}$ and receives (com.setup.end, sid, $O K$ ) from $\mathcal{F}_{\mathrm{NIC}}$.

- $\mathcal{P}$ initializes a counter $c p \leftarrow 0$ and stores $($ sid, Tbl, $c p)$.

- $\mathcal{P}$ outputs (zkc.setup.end, sid).

Fig. 19. Construction $\Pi_{\mathrm{ZKC}}^{R}$ : interface zkc.setup 
2. On input (zkc.prove.ini, sid, wit ${ }_{i}$, ins), $\mathcal{P}$ and $\mathcal{V}$ do the following:

- $\mathcal{P}$ aborts if $($ sid, $\mathrm{Tbl}, c p)$ is not stored or if $\left(\right.$ wit $_{i}$, ins $) \notin R$.

- $\mathcal{P}$ increments the counter $c p$ in $(s i d$, Tbl, $c p)$.

- $\mathcal{P}$ retrieves the bijective function $f$ in $R_{c}$ and computes $i \leftarrow f\left(\right.$ wit $\left._{i}\right)$. $\mathcal{P}$ sends (com.commit.ini, sid, $i$ ) to $\mathcal{F}_{\mathrm{NIC}}$ and receives (com.commit.end, sid, com, open) from $\mathcal{F}_{\mathrm{NIC}}$.

- $\mathcal{P}$ retrieves the entry $\left[w_{i} t_{i}, c t_{i}\right]$ in Tbl and sets $v_{1} \leftarrow c t_{i}$ and $v_{2} \leftarrow c t_{i}+1$. $\mathcal{P}$ aborts if $v_{2}>c t_{\max }$. Else, $\mathcal{P}$ sends (com.commit.ini, sid, $v_{1}$ ) to $\mathcal{F}_{\mathrm{NIC}}$ and receives (com.commit.end, sid, com $_{1}$, open op $_{1}$ ) from $\mathcal{F}_{\mathrm{NIC}} \mathcal{P}$ sends the message (com.commit.ini, sid, $v_{2}$ ) to $\mathcal{F}_{\text {NIC }}$ and receives (com.commit.end, sid, com $_{2}$, open ${ }_{2}$ ) from $\mathcal{F}_{\text {NIC. }}$

- $\mathcal{P}$ sets wit $_{c} \leftarrow\left(\right.$ wit $_{i}, i$, open, $v_{1}$, open $_{1}, v_{2}$, open $\left._{2}\right)$.

- $\mathcal{P}$ parses com as $\left(\mathrm{com}^{\prime}\right.$, parcom, COM.Verify).

- $\mathcal{P}$ parses $\mathrm{com}_{1}$ as $\left(\mathrm{com}_{1}^{\prime}\right.$, parcom $\left._{1}, \mathrm{COM} . V e r i f y_{1}\right)$.

- $\mathcal{P}$ parses $\mathrm{com}_{2}$ as $\left(\right.$ com $_{2}^{\prime}$, parcom $_{2}$, COM.Verify $\left._{2}\right)$.

- $\mathcal{P}$ sets ins $_{c} \leftarrow\left(\right.$ ins, parcom, com $^{\prime}$, com $_{1}^{\prime}$, com $\left._{2}^{\prime}, c p\right)$.

- $\mathcal{P}$ stores $\left(c p\right.$, wit $_{c}$, ins $_{c}$, read $)$.

- $\mathcal{P}$ sends (zk.prove.ini, sid, wit , $_{\text {, ins }}$ ) to $\mathcal{F}_{\mathrm{ZK}}^{R_{c}}$.

- $\mathcal{V}$ receives (zk.prove.end, sid, ins $s_{c}$ ) from $\mathcal{F}_{\mathrm{ZK}}^{R_{c}}$.

- $\mathcal{V}$ parses ins $_{c}$ as (ins, parcom, com', $\left.c o m_{1}^{\prime}, c o m_{2}^{\prime}, c p\right)$.

- $\mathcal{V}$ aborts if $c p \neq c v+1$ or if there exists $i n s_{c}^{\prime}$ already stored.

- $\mathcal{V}$ sets com $\leftarrow\left(\mathrm{com}^{\prime}\right.$, parcom, COM.Verify $), \operatorname{com}_{1} \leftarrow\left(\mathrm{com}_{1}^{\prime}\right.$, parcom, COM.Verify) and $\mathrm{com}_{2} \leftarrow\left(\operatorname{com}_{2}^{\prime}\right.$, parcom, COM.Verify). (COM.Verify is part of the description of the relation $R_{c}$.)

- $\mathcal{V}$ sends the message (com.validate.ini, sid, com) to $\mathcal{F}_{\text {NIC }}$ and receives (com.validate.end, sid, $b$ ) from $\mathcal{F}_{\text {NIC }}$. $\mathcal{V}$ sends (com.validate.ini, sid, com $\left._{1}\right)$ to $\mathcal{F}_{\mathrm{NIC}}$ and receives (com.validate.end, sid, $\left.b_{1}\right)$ from $\mathcal{F}_{\mathrm{NIC}}$. $\mathcal{V}$ sends (com.validate.ini, sid, $\operatorname{com}_{2}$ ) to $\mathcal{F}_{\mathrm{NIC}}$ and receives (com.validate.end, sid, $\left.b_{2}\right)$ from $\mathcal{F}_{\text {NIC }}$. If $b=b_{1}=b_{2}=1$ is not fulfilled, $\mathcal{V}$ aborts.

- $\mathcal{V}$ stores $i n s_{c}$.

- $\mathcal{V}$ sets $\operatorname{sid}_{\mathrm{SMT}} \leftarrow\left(\mathcal{V}, \mathcal{P}\right.$, sid $\left.^{\prime}\right)$ and sends (smt.send.ini, $\left.\operatorname{sid}_{\mathrm{SMT}},\langle c p, \mathrm{read}\rangle\right)$ to $\mathcal{F}_{\mathrm{SMT}}$.

- $\mathcal{P}$ receives (smt.send.end, $\operatorname{sid}_{\mathrm{SMT}},\langle c p$, read $\rangle$ ) from $\mathcal{F}_{\mathrm{SMT}}$.

- $\mathcal{P}$ aborts if $\left(c p\right.$, wit $_{c}$, ins $_{c}$, read $)$ is not stored.

- $\mathcal{P}$ parses $i n s_{c}$ as (ins, parcom, com' com $\left._{1}^{\prime}, \mathrm{com}_{2}^{\prime}, c p\right)$.

- $\mathcal{P}$ parses wit $_{c}$ as $\left(\right.$ wit $_{i}, i$, open, $v_{1}$, open $_{1}, v_{2}$, open $\left._{2}\right)$.

- $\mathcal{P}$ sets com $\leftarrow\left(\mathrm{com}^{\prime}\right.$, parcom, COM.Verify $), \operatorname{com}_{1} \leftarrow\left(\mathrm{com}_{1}^{\prime}\right.$, parcom, COM.Verify $)$ and com $_{2} \leftarrow\left(\right.$ com $_{2}^{\prime}$, parcom, COM.Verify). (COM.Verify is part of the description of the relation $R_{c}$.)

- $\mathcal{P}$ replaces $\left(c p, w_{i} t_{c}\right.$, ins $_{c}$, read) by $\left(c p\right.$, wit $_{c}$, ins $_{c}$, write).

- $\mathcal{P}$ sends (cd.read.ini, sid, com, $i$, open, com $_{1}, v_{1}$, open 1 ) to $\mathcal{F}_{\mathrm{CD}}$.

- $\mathcal{V}$ receives (cd.read.end, sid, com, com $_{1}$ ) from $\mathcal{F}_{\mathrm{CD}}$.

- $\mathcal{V}$ aborts if $i n s_{c}$ is not stored.

- $\mathcal{V}$ parses ins $_{c}$ as (ins, parcom, com ${ }^{\prime}$, com $_{1}^{\prime}$, com $\left._{2}^{\prime}, c p\right)$.

- $\mathcal{V}$ compares the commitments received from $\mathcal{F}_{\mathrm{CD}}$ with those in $i n s_{c}$. If they are not equal, $\mathcal{V}$ aborts.

- $\mathcal{V}$ sets $\operatorname{sid}_{\mathrm{SMT}} \leftarrow\left(\mathcal{V}, \mathcal{P}\right.$, sid $\left.^{\prime}\right)$ and sends (smt.send.ini, $\operatorname{sid}_{\mathrm{SMT}},\langle c p$, write $\left.\rangle\right)$ to $\mathcal{F}_{\mathrm{SMT}}$.

- $\mathcal{P}$ receives (smt.send.end, $s i d_{\mathrm{SMT}},\langle c p$, write $\rangle$ ) from $\mathcal{F}_{\mathrm{SMT}}$.

- $\mathcal{P}$ aborts if $\left(c p\right.$, wit $_{c}$, ins $_{c}$, write $)$ is not stored.

- $\mathcal{P}$ parses ins $_{c}$ as (ins, parcom, com ${ }^{\prime}$ com $_{1}^{\prime}$, com $\left._{2}^{\prime}, c p\right)$.

- $\mathcal{P}$ parses wit $_{c}$ as $\left(\right.$ wit $_{i}, i$, open, $v_{1}$, open $_{1}, v_{2}$, open $\left._{2}\right)$.

- $\mathcal{P}$ deletes the record $\left(c p\right.$, wit $_{c}$, ins $_{c}$, write).

- $\mathcal{P}$ sends (cd.write.ini, sid, com, $i$, open, com $_{2}, v_{2}$, open ${ }_{2}$ ) to $\mathcal{F}_{\mathrm{CD}}$.

- $\mathcal{V}$ receives (cd.write.end, sid, com, $\operatorname{com}_{2}$ ) from $\mathcal{F}_{\mathrm{CD}}$.

- $\mathcal{V}$ aborts if $i n s_{c}$ is not stored.

- $\mathcal{V}$ parses ins $_{c}$ as (ins, parcom, com $^{\prime}$, com $_{1}^{\prime}$, com $\left._{2}^{\prime}, c p\right)$.

- $\mathcal{V}$ compares the commitments received from $\mathcal{F}_{\mathrm{CD}}$ with those in $i n s_{c}$. If they are not equal, $\mathcal{V}$ aborts.

- $\mathcal{V}$ increments $c v$ in $(s i d, c v)$ and deletes the record $i n s_{c}$.

- $\mathcal{V}$ outputs (zkc.prove.end, sid, ins).

Fig. 20. Construction $\Pi_{\mathrm{ZKC}}^{R}$ : interface zkc.prove 
3. On input (zkc.count.ini, sid, wit $\left.{ }_{i}\right), \mathcal{P}$ and $\mathcal{V}$ do the following:

- $\mathcal{P}$ aborts if $($ sid $, \mathrm{Tbl}, c p)$ is not stored or if wit $_{i} \notin\left(\right.$ wit $_{1}, \ldots$, wit $\left._{N}\right)$.

- $\mathcal{P}$ retrieves the bijective function $f$ in $R_{c}$ and computes $i \leftarrow f\left(w_{i} t_{i}\right)$. $\mathcal{P}$ sends (com.commit.ini, sid, $i$ ) to $\mathcal{F}_{\text {NIC }}$ and receives (com.commit.end, sid, com, open) from $\mathcal{F}_{\text {NIC. }}$.

- $\mathcal{P}$ retrieves the entry $\left[\right.$ wit $\left._{i}, c t_{i}\right]$ in Tbl and sets $v_{1} \leftarrow c t_{i}$. $\mathcal{P}$ sends the message (com.commit.ini, sid, $v_{1}$ ) to $\mathcal{F}_{\mathrm{NIC}}$ and receives (com.commit.end, sid, com $_{1}$, open $_{1}$ ) from $\mathcal{F}_{\text {NIC. }}$.

- $\mathcal{P}$ stores (com, i, open, com $_{1}, v_{1}$, open $\left._{1}\right)$.

- $\mathcal{P}$ sends (cd.read.ini, sid, com, $i$, open, com $_{1}, v_{1}$, open 1 ) to $\mathcal{F}_{\mathrm{CD}}$.

- $\mathcal{V}$ receives (cd.read.end, sid, com, com 1 ) from $\mathcal{F}_{\mathrm{CD}}$.

- $\mathcal{V}$ stores $\left(\mathrm{com}, \mathrm{com}_{1}\right)$.

- $\mathcal{V}$ sets $\operatorname{sid}_{\mathrm{SMT}} \leftarrow\left(\mathcal{V}, \mathcal{P}\right.$, sid $\left.{ }^{\prime}\right)$ and sends the message (smt.send.ini, sid $\left.{ }_{\mathrm{SMT}},\left\langle c o m, \operatorname{com}_{1}\right\rangle\right)$ to $\mathcal{F}_{\mathrm{SMT}}$.

- $\mathcal{P}$ receives (smt.send.end, $\left.\operatorname{sid}_{\mathrm{SMT}},\left\langle c o m, \operatorname{com}_{1}\right\rangle\right)$ from $\mathcal{F}_{\mathrm{SMT}}$.

- $\mathcal{P}$ aborts if $\left(\mathrm{com}^{\prime}, i\right.$, open, $\mathrm{com}_{1}^{\prime}, v_{1}$, open $\left._{1}\right)$ such that $\mathrm{com}^{\prime}=\mathrm{com}$ and $\mathrm{com}_{1}^{\prime}=\mathrm{com}_{1}$ is not stored.

- $\mathcal{P}$ deletes the record (com, $i$, open, com $_{1}, v_{1}$, open $\left._{1}\right)$.

- $\mathcal{P}$ sends (smt.send.ini, sid, $\left\langle\right.$ com, $i$, open, com $_{1}, v_{1}$, open $\left.\left._{1}\right\rangle\right)$ to $\mathcal{F}_{\mathrm{SMT}}$.

- $\mathcal{V}$ receives (smt.send.end, sid, $\left\langle\right.$ com, $i$, open, com $_{1}, v_{1}$, open 1$\left.\rangle\right)$ from the functionality $\mathcal{F}_{\mathrm{SMT}}$.

- $\mathcal{V}$ aborts if $\left(\mathrm{com}^{\prime}, \mathrm{com}_{1}^{\prime}\right)$ such that $\mathrm{com}^{\prime}=\mathrm{com}$ and $\mathrm{com}_{1}^{\prime}=\mathrm{com}_{1}$ is not stored.

- $\mathcal{V}$ sends (com.verify.ini, sid, com, $i$, open) to $\mathcal{F}_{\text {NIC }}$ and receives the message (com.verify.end, sid, b) from $\mathcal{F}_{\text {NIC. }} \mathcal{V}$ sends the message (com.verify.ini, sid, com, $v_{1}$, open $_{1}$ ) to $\mathcal{F}_{\text {NIC }}$ and receives (com.verify.end, sid, $b_{1}$ ) from $\mathcal{F}_{\text {NIC. }}$. If $b=b_{1}=1$ is not fulfilled, $\mathcal{V}$ aborts.

- $\mathcal{V}$ uses the inverse function of $f$ to compute the witness value wit ${ }_{i}$ associated with $i$.

- $\mathcal{V}$ deletes the record $\left(c o m, c_{1} m_{1}\right)$.

- $\mathcal{V}$ outputs (zkc.count.end, sid, wit $\left.{ }_{i}, v_{1}\right)$.

Fig. 21. Construction $\Pi_{\mathrm{ZKC}}^{R}$ : interface zkc.count

message to the adversary if the functionality sends the abortion message to a corrupt party.

\section{A. Security Analysis of Construction $\Pi_{\mathrm{ZKC}}^{R}$ when $\mathcal{P}$ is Corrupt.}

We describe the simulator $\mathcal{S}$ for the case in which the prover is corrupt. Basically, $\mathcal{S}$ simulates the protocol by running copies of the ideal functionalities and of protocol $\Pi_{\mathrm{ZKC}}^{R}$ for the verifier.

- $\mathcal{S}$ initializes an empty table Tbl with entries $\left[\right.$ wit $\left._{i}, 0\right]$ for $i=1$ to $N$.

- When receiving a message from $\mathcal{A}, \mathcal{S}$ uses the first field of the message to associate the message to one of the ideal functionalities $\mathcal{F}_{\mathrm{CD}}, \mathcal{F}_{\mathrm{ZK}}^{R_{c}}, \mathcal{F}_{\mathrm{SMT}}$ or $\mathcal{F}_{\mathrm{NIC}}$ and runs a copy of the corresponding functionality on input that message.

- When the copy of any of the functionalities $\mathcal{F}_{\mathrm{CD}}, \mathcal{F}_{\mathrm{ZK}}^{R_{c}}$, $\mathcal{F}_{\mathrm{SMT}}$ and $\mathcal{F}_{\text {NIC }}$ sends a message to the prover or to the simulator, $\mathcal{S}$ forwards the output of the functionality to $\mathcal{A}$.

- When the copy of any of the functionalities $\mathcal{F}_{\mathrm{CD}}, \mathcal{F}_{\mathrm{ZK}}^{R_{c}}$, $\mathcal{F}_{\mathrm{SMT}}$ and $\mathcal{F}_{\mathrm{NIC}}$ sends a message to the verifier, the simulator runs protocol $\Pi_{\mathrm{ZKC}}^{R}$ for the verifier on input that message.

- When protocol $\Pi_{\mathrm{ZKC}}^{R}$ for the verifier sends a message to any of the functionalities $\mathcal{F}_{\mathrm{CD}}, \mathcal{F}_{\mathrm{ZK}}^{R_{c}}, \mathcal{F}_{\mathrm{SMT}}$ and $\mathcal{F}_{\mathrm{NIC}}, \mathcal{S}$ runs the copy of the respective functionality on input that message.
- When protocol $\Pi_{\mathrm{ZKC}}^{R}$ for the verifier outputs the message (zkc.prove.end, sid, ins), $\mathcal{S}$ retrieves the message (zk.prove.ini, sid, wit , $_{\text {ins }}$ ) sent by $\mathcal{A}$ such that ins $\in$ ins $_{c} . \mathcal{S}$ parses wit $_{c}$ as $\left(\right.$ wit $_{i}, i$, open, $_{1}$, open $_{1}, v_{2}$, open $\left._{2}\right) . \mathcal{S}$ increments the counter in the entry in Tbl that corresponds to wit $i$ and sends (zkc.prove.ini, sid, wit $_{i}$, ins) to $\mathcal{F}_{\mathrm{ZKC}}^{R}$. When $\mathcal{F}_{\mathrm{ZKC}}^{R}$ sends (zkc.prove.sim, sid, qid, ins) to $\mathcal{S}, \mathcal{S}$ sends (zkc.prove.rep, sid, qid) to $\mathcal{F}_{\mathrm{ZKC}}^{R}$.

- When protocol $\Pi_{\mathrm{ZKC}}^{R}$ for the verifier outputs the message (zkc.count.end, sid, wit $\left.{ }_{i}, v_{1}\right), \mathcal{S}$ checks that there is an entry $\left[\right.$ wit $\left._{i}, v_{1}\right]$ in Tbl. If the entry does not exist, $\mathcal{S}$ outputs failure. Otherwise, $\mathcal{S}$ sends (zkc.count.ini, sid, wit $_{i}$ ) to $\mathcal{F}_{\mathrm{ZKC}}^{R}$. When $\mathcal{F}_{\mathrm{ZKC}}^{R}$ sends (zkc.count.sim, sid, qid) to $\mathcal{S}, \mathcal{S}$ sends (zkc.count.rep, sid, qid) to $\mathcal{F}_{\mathrm{ZKC}}^{R}$.

- When protocol $\Pi_{\mathrm{ZKC}}^{R}$ for the verifier outputs an abortion message, $\mathcal{S}$ picks a random qid and sends (zkc.count.rep, sid, qid) to $\mathcal{F}_{\mathrm{ZKC}}^{R}$, so that $\mathcal{F}_{\mathrm{ZKC}}^{R}$ sends an abortion message to the dummy verifier.

Theorem N.2: When the prover $\mathcal{P}$ is corrupt, the construction $\Pi_{\mathrm{ZKC}}^{R}$ securely realizes $\mathcal{F}_{\mathrm{ZKC}}^{R}$ in the $\mathcal{F}_{\mathrm{CD}}, \mathcal{F}_{\mathrm{ZK}}^{R_{c}}, \mathcal{F}_{\mathrm{SMT}}$, and $\mathcal{F}_{\text {NIC }}$-hybrid model.

Proof Sketch. Our simulator $\mathcal{S}$ is indistinguishable from the real world protocol because it runs as the real-world protocol, except when it outputs failure. The probability that $\mathcal{S}$ outputs failure is negligible thanks to the security properties ensured by the functionalities $\mathcal{F}_{\mathrm{CD}}, \mathcal{F}_{\mathrm{ZK}}^{R_{c}}, \mathcal{F}_{\mathrm{SMT}}$, and $\mathcal{F}_{\mathrm{NIC}}$. Concretely, 


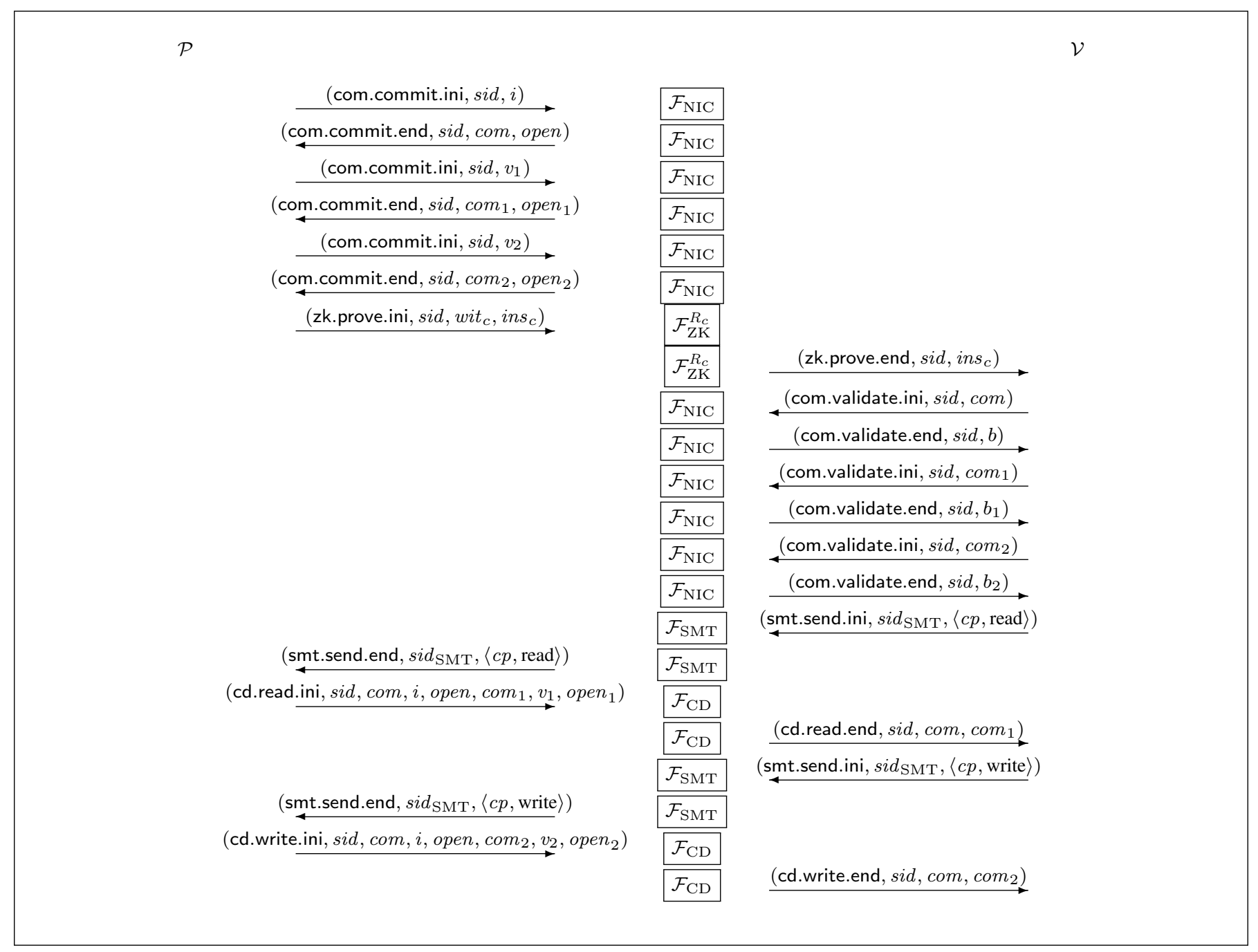

Fig. 22. Construction $\Pi_{\mathrm{ZKC}}^{R}$ : interface zkc.prove.

$\mathcal{F}_{\mathrm{NIC}}$ ensures that commitments are binding. $\mathcal{F}_{\mathrm{ZK}}^{R_{c}}$ ensures that the witness wit $_{c}$ and instance $i n s_{c}$ given by the prover satisfy the relation $R_{c}$. The relation $R_{c}$ guarantees that the prover knows a witness wit $_{i}$ and an instance ins that satisfy the relation $R$. Additionally, it associates wit $i$ with a position $i$ committed to in com and it shows that two commitments $\mathrm{com}_{1}$ and $\operatorname{com}_{2}$ commit to values $v_{1}$ and $v_{2}$ such that $v_{2}$ increments $v_{1} . \mathcal{F}_{\mathrm{CD}}$ ensures that com and $\operatorname{com}_{1}$ commit a position $i$ and a value $v_{1}$ that was previously stored, and that $\operatorname{com}_{2}$ commits to the new value $v_{2}$ that is stored at position $i$.

\section{B. Security Analysis of Construction $\Pi_{\mathrm{ZKC}}^{R}$ when $\mathcal{V}$ is Corrupt.}

We describe the simulator $\mathcal{S}$ for the case in which the verifier is corrupt. Basically, $\mathcal{S}$ simulates the protocol by running copies of the ideal functionalities and of protocol $\Pi_{\mathrm{ZKC}}^{R}$ for the prover. In contrast to the case of a corrupt prover, we need to modify $\Pi_{\mathrm{ZKC}}^{R}$ and the code of the ideal functionalities in several ways, which we detail below.
- When $\mathcal{F}_{\mathrm{ZKC}}^{R}$ sends a message (zkc.prove.sim, sid, qid, ins), $\mathcal{S}$ sends the message (zkc.prove.rep, sid, qid) to $\mathcal{F}_{\mathrm{ZKC}}^{R}$

- When $\mathcal{F}_{\mathrm{ZKC}}^{R}$ sends a message (zkc.count.sim, sid, qid), $\mathcal{S}$ sends (zkc.count.rep, sid, qid) to $\mathcal{F}_{\mathrm{ZKC}}^{R}$.

- When $\mathcal{F}_{\mathrm{ZKC}}^{R}$ sends a message (zkc.prove.end, sid, ins), $\mathcal{S}$ runs the protocol $\Pi_{\mathrm{ZKC}}^{R}$ for the prover on input (zkc.prove.ini, sid, $\perp$, ins). The difference with respect to the real world protocol is that wit $i_{i}$ is replaced by $\perp$, and that the elements $\left(i, v_{1}, v_{2}\right)$ to set the witness witc are taken randomly.

- When $\mathcal{F}_{\mathrm{ZKC}}^{R}$ sends a message (zkc.count.end, sid, wit ${ }_{i}$, $\left.c t_{i}^{\prime}\right), \mathcal{S}$ runs the protocol $\Pi_{\mathrm{ZKC}}^{R}$ for the prover on that input.

- When the protocol $\Pi_{\mathrm{ZKC}}^{R}$ sends a message to any of the ideal functionalities $\mathcal{F}_{\mathrm{CD}}, \mathcal{F}_{\mathrm{ZK}}^{R_{c}}, \mathcal{F}_{\mathrm{SMT}}$ and $\mathcal{F}_{\mathrm{NIC}}, \mathcal{S}$ runs the copy of the respective functionality on input that message. In the code of $\mathcal{F}_{\mathrm{ZK}}^{R_{c}}$, the check $\left(\right.$ wit $_{c}$, ins $\left._{c}\right) \in R_{c}$ is removed. In the code of $\mathcal{F}_{\mathrm{CD}}$, the check $\left[i, v_{1}\right] \in \mathrm{Tbl}$ 


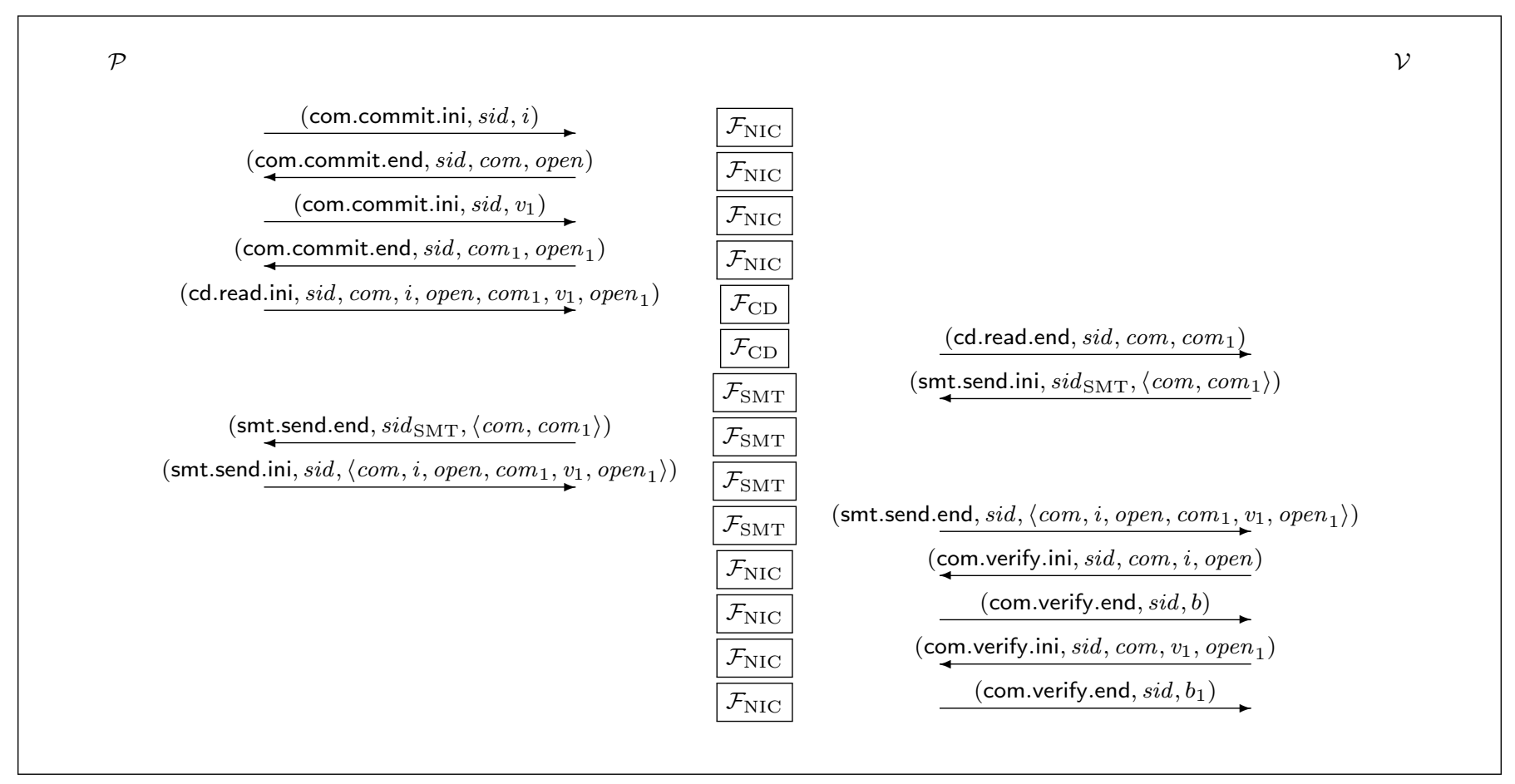

Fig. 23. Construction $\Pi_{\mathrm{ZKC}}^{R}$ : interface zkc.count.

is removed.

- When the copy of any of the functionalities $\mathcal{F}_{\mathrm{CD}}, \mathcal{F}_{\mathrm{ZK}}^{R_{c}}$, $\mathcal{F}_{\mathrm{SMT}}$ and $\mathcal{F}_{\mathrm{NIC}}$ sends a message to the verifier or to the simulator, $\mathcal{S}$ forwards the output of the functionality to $\mathcal{A}$.

- When the copy of any of the functionalities $\mathcal{F}_{\mathrm{CD}}, \mathcal{F}_{\mathrm{ZK}}^{R_{c}}$, $\mathcal{F}_{\mathrm{SMT}}$ and $\mathcal{F}_{\mathrm{NIC}}$ sends a message to the prover, the simulator runs protocol $\Pi_{\mathrm{ZKC}}^{R}$ for the prover on input that message.

- When receiving a message from $\mathcal{A}$, our simulator runs a copy of the corresponding functionality $\mathcal{F}_{\mathrm{CD}}, \mathcal{F}_{\mathrm{ZK}}^{R_{c}}, \mathcal{F}_{\mathrm{SMT}}$ and $\mathcal{F}_{\mathrm{NIC}}$ on input that message.

Theorem N.3: When the verifier $\mathcal{V}$ is corrupt, the construction $\Pi_{\mathrm{ZKC}}^{R}$ securely realizes $\mathcal{F}_{\mathrm{ZKC}}^{R}$ in the $\mathcal{F}_{\mathrm{CD}}, \mathcal{F}_{\mathrm{ZK}}^{R_{c}}, \mathcal{F}_{\mathrm{SMT}}$, and $\mathcal{F}_{\text {NIC-hybrid model. }}$

Proof Sketch. We show that our simulator $\mathcal{S}$ is indistinguishable from the real-world protocol. $\mathcal{S}$ replaces $w_{i} t_{i}$ by $\perp$ and $\left(i, v_{1}, v_{2}\right)$ by random values in the correct domains. However, $\mathcal{A}$ never receives any information about those values. The simulator also removes the checks $\left(w_{i} t_{c}, i n s_{c}\right) \in R_{c}$ in the code of $\mathcal{F}_{\mathrm{ZK}}^{R_{c}}$ and $\left[i, v_{1}\right] \in \mathrm{Tbl}$ in the code of $\mathcal{F}_{\mathrm{CD}}$. However, the commitments com, com 1 and com $_{2}$ and the instance $i n s_{c}$ are indistinguishable from the real-world ones, and the correct values wit $_{i}$ and $c t_{i}^{\prime}$ are used when they are revealed by $\mathcal{F}_{\mathrm{ZKC}}^{R}$.

\section{APPENDIX O RELATED WORK}

Commitments and ZK proofs of shuffles. Many protocols (e.g. [1], [10], [11]) use commitment schemes to maintain a database between a prover $\mathcal{P}$ and a verifier $\mathcal{V}$. However, commitments on their own are not adequate to realize $\mathcal{F}_{\mathrm{CD}}$ because they do not allow $\mathcal{P}$ to hide the positions read from or written into the database. ZK proofs of shuffles [14] can be used to shuffle the commitments in order to hide the positions read or written. A construction using commitments along with proofs of shuffles could realize $\mathcal{F}_{\mathrm{CD}}$, but less efficiently than a construction based on vector commitments.

Accumulators. A cryptographic accumulator [31] allows one to represent a set $X$ succinctly as a single accumulator value $A$. It also provides a method to prove succinctly that an element $x$ belongs to $X$ to any party that holds $A$. This method consists in computing a witness $W$ whose size is independent of the size $|X|$ of the set. Soundness (or collision-freeness) guarantees that it is infeasible to prove that $x \in X$ if $x \notin X$.

Since their introduction, a number of accumulator schemes have been proposed (see [42] for a comprehensive review). Some accumulator schemes are trapdoorless, i.e., the party that generates the parameters of the scheme does not learn any trapdoor [43]-[46]. However, most practical accumulator schemes use a trusted setup [42], [47]-[51]. In the following, we will discuss schemes that use a trusted setup.

Extensions to accumulator schemes include schemes that support non-membership witnesses [48], [51], [52], i.e. schemes where it is possible to compute a proof that $x \notin X$, which are referred to as universal. Some schemes, referred to as dynamic, allow efficient updates [47], [48], [50], i.e. the accumulator value $A$ can be updated to add or remove elements from the accumulated set with cost independent of $|X|$, and witnesses can also be updated with cost independent of $|X|$. For many applications of accumulators, e.g. revocation of 
anonymous credentials, accumulator schemes are extended with zero-knowledge proofs of knowledge of witnesses to prove (non-)membership.

Most accumulator schemes do not hide the accumulated set, i.e., the value $A$ does not hide the accumulated set $X$. Recently, an indistinguishability definition [42] and a simulation-based definition [51] of the property of hiding the accumulated set were proposed. The definition in [51], referred to as zeroknowledge accumulators, is stronger. The main difference is that, in [42], the updates to the accumulated set are not guaranteed to be hidden, while in [51] this is guaranteed. The reason is that, in [42], although $A$ is computed initially through a randomized algorithm, subsequent updates are computed deterministically, whereas in [51] both the initial computation of $A$ and subsequent updates are computed with randomized algorithms.

Our construction for a committed database uses a vector commitment scheme with efficient updates. The main difference between vector commitments and accumulators is that, while accumulators allow for committing to a set, vector commitments allow for committing to a vector of messages, where each message is committed at a specific position. This allows the construction of an updatable committed database where it is also possible to prove statements about the position where a message is written or read.

The vector commitment scheme with efficient updates that we use provides randomized algorithms both to compute a vector commitment and to update it. This idea resembles the construction of zero-knowledge accumulators in [51]. Our definition of the hiding property for a vector commitment with updates requires that an updated vector commitment cannot be distinguished from a freshly generated one. We note that, in our construction for a committed database, the prover never reveals commitment openings (or witnesses) to the verifier, and thus we do not require a hiding property that involves commitment openings. Nevertheless, we think that the vector commitment scheme with efficient updates that we use could fulfill a simulation-based definition of the hiding property akin to the zero-knowledge definition for accumulators provided in [51].

We use an instantiation of a vector commitment scheme with efficient updates based on the Diffie-Hellman exponent (DHE) assumption. This instantiation resembles the accumulator scheme in [50]. The scheme in [42] and in [51] extend the scheme in [49], which is based on the strong bilinear Diffie-Hellman (SBDH) assumption. (In the paragraph about vector commitments, we explain why we choose a scheme based on DHE.) We think that the techniques to re-randomize commitments and witnesses used to construct the vector commitment with efficient updates based on DHE can be applied to extend the accumulator scheme [50] in order to obtain a zero-knowledge accumulator based on DHE.

Vector Commitments. Vector commitments [6], [7] allow a committer to commit to a vector of values. Subsequently, the committer is able to open the commitment to a vector component. Both the size of the commitment and the size of an opening are independent of the length of the committed vector.

Vector commitments (VC) are defined in [7]. This definition is for non-hiding vector commitments with updates. To obtain hiding vector commitments, it is suggested to compose a nonhiding vector commitment scheme with a standard commitment scheme. Two constructions of non-hiding vector commitments are given based on the $\mathrm{CDH}$ and RSA assumptions. In [6], a construction of mercurial vector commitments based on the Diffie-Hellman Exponent (DHE) assumption is proposed. ${ }^{1}$ This construction leads to constructions of non-hiding and hiding vector commitments based on DHE, which were used in, e.g., [32]-[34].

Our committed database uses as building block any hiding vector commitment scheme with updates, along with zeroknowledge proofs that a vector component is being read or written. To instantiate our committed database, we use a construction of hiding vector commitments with updates based on the DHE assumption along with the corresponding zeroknowledge proofs for reading and writing. In this construction, the size of the public parameters is linear in the maximum vector length, and updates of commitments and of openings can be computed with cost linear in the number of updated elements but independent of the vector length. In comparison, in a hiding $\mathrm{VC}$ construction from $\mathrm{CDH}$, the size of the public parameters would be quadratic (the advantage would be to use a more standard assumption).

Recently, in [35], subvector commitments (SVC) are proposed. In SVC, a commitment can be opened to a set of positions such that the size of the witness does not depend on the size of the set. A construction for SVC secure under the cube Diffie-Hellman assumption is given, in which the public parameter size grows quadratically with the vector length. $\mathcal{F}_{\mathrm{CD}}$ and our applications for it in Section VIII only require to open one vector component at a time. SVC can be used to construct a variant of $\mathcal{F}_{\mathrm{CD}}$ where several positions are read or written simultaneously. Nevertheless, we note that, despite that SVC provides witnesses of size independent of the number of positions opened, the entire witness of read or write proofs would still grow with the number of positions, and thus the efficiency of those proofs would not be independent of the set size. In [35], [36], constructions for SVC based on groups of hidden order are proposed that are better suited for bit vectors. Polynomial and functional commitments. Polynomial commitments allow a committer to commit to a polynomial and open the commitment to an evaluation of the polynomial. They can be used as vector commitments by committing to

\footnotetext{
${ }^{1}$ Informally speaking, mercurial commitments are commitments where the binding property is slightly relaxed in that the committer is allowed to softly open a commitment and say "if the commitment can be opened at all, then it opens to that message". Upon committing, the sender has to decide whether the commitment will be a hard commitment, that can be hard/soft-opened to only one message, or a soft one that can be soft-opened to any arbitrary message without committing the sender to a specific one. Unlike soft commitments that cannot be hard-opened, hard commitments can be opened either in the soft or the hard manner but soft openings can never contradict hard ones. In addition, hard and soft commitments should be computationally indistinguishable.
} 
a polynomial that interpolates the vector to be committed. In [12], a construction of polynomial commitments from the $\mathrm{SDH}$ assumption is proposed, which has the disadvantage that efficient updates cannot be computed without knowledge of the trapdoor. A further generalization of vector commitments and polynomial commitments are functional commitments [13].

Zero-Knowledge Data Structures. Zero-Knowledge Sets (ZKS) [15] allow a prover $\mathcal{P}$ to commit to a set $X$ and to subsequently prove to a verifier $\mathcal{V}$ (non-)membership of an element $x$ in $X$. Zero-Knowledge Databases (ZKDB) are similar to ZKS but each element $x \in X$ is associated with a value $v$, in such a way that a proof that $x \in X$ reveals $v$ to $\mathcal{V}$ Both ZKS and ZKDB are two-party protocols between $\mathcal{P}$ and $\mathcal{V}$. Security for $\mathcal{V}$ requires that an adversarial $\mathcal{P}$ is not able to prove $x \in X$ if $x \notin X$ (and vice versa), while zero-knowledge requires that proofs of (non-)membership reveal nothing else beyond (non-)membership, not even the size of the set.

Zero-knowledge sets were introduced in [15]. In [16], ZKS were constructed from trapdoor mercurial commitments and collision resistant hash functions. In [53], it was shown that (trapdoor) mercurial commitments can be constructed from oneway functions. These ZKS constructions use variants of Merkle trees where nodes are filled with mercurial commitments to their children. Because of the need to hide any information on the size of $X$, the depth $k$ of the tree, which determines an upper bound on the size of the committable set, must fulfill $2^{k}>>|X|$. As a consequence, constructions are inefficient because proofs of (non-)membership grow linearly with the tree depth.

ZKS with short proofs for membership were proposed in [17], while short proofs for membership and non-membership are attained in [6]. The latter ZKS construction is the one based on mercurial vector commitments from DHE. In [7], it is proven that mercurial vector commitments can be instantiated from (non-hiding) vector commitments and trapdoor mercurial commitments. The constructions of vector commitments from the RSA and CDH assumptions given in [7] lead to ZKS with short proofs from standard assumptions. In [19], a construction for zero-knowledge lists (ZKL) is proposed, where a list is defined as an ordered set. The construction in [19] uses a ZKS and homomorphic commitments as building blocks. In most of the constructions above, the data structure is not updatable. Updatable ZKDB were first proposed in [18]. In [7], a construction for updatable ZKDB based on updatable vector commitments and trapdoor mercurial commitments is proposed.

There are several differences between our committed database and previous work. First, our committed database is updatable, which was only considered in [7], [18]. Second, our committed database is oblivious. $\mathcal{P}$ proves in zero-knowledge that a pair of commitments commit to a position and value that are stored in the database. In contrast, in previous constructions, $\mathcal{P}$ reveals a position and a value along with a proof that the position and the value are stored in the database. The obliviousness property allows our committed database to be used as building block in applications that protect the privacy of $\mathcal{P}$, because $\mathcal{P}$ could choose to open the commitments, but could also prove statements in $\mathrm{ZK}$ about the committed position and value without revealing them.

From a definitional point of view, security definitions given in previous works are not in the UC model and a mechanism to integrate modularly ZKS or ZKDB as building blocks of other protocols is not given. Our functionality for a committed database allows the security analysis of $\mathrm{ZK}$ data structures in a composable framework, which will facilitate the modular design and analysis of protocols that use them as a building block.

Another key difference between our definition and construction and the definitions and constructions in the abovementioned works is that we do not require the size of the database to be hidden. In fact, our construction reveals an upper bound of the database size that could be similar to the actual size of the committed database. Thanks to this relaxation, we can offer a construction for a committed database that is more efficient than the above constructions for ZKS or ZKDB. Instead of using a variant of a Merkle tree, in our construction, a single vector commitment is used to commit to the database. This leads to more efficient proofs and updates. This relaxation of the zero-knowledge property is not relevant for our applications for a committed database. In this respect, our construction for a committed database is similar to the constructions for "nearly" ZKS and ZKDB given in [12] In [12], ZKS and ZKDB that do not hide the size of the set or database are provided based on polynomial commitments. However, this "nearly" ZKS and ZKDB constructions based on the SDH assumption are not updatable and, moreover, extending them with efficient updates is not possible (see discussion on the paragraph about vector commitments). In fact, as pointed out in [37], when hiding the size of the database is required, for any construction that uses a non-interactive commitment phase (as is the case in the ZKS and ZKDB constructions cited above), black-box extraction of the database by the simulator in the security proof is not possible. In [37], a secure committed database where the database size is hidden is defined in the UC model, and a construction that uses an interactive commitment phase is proposed. As a consequence of needing to hide the database size, the construction in [37] is also less efficient than ours. Also, their ideal functionality does not facilitate modular design, and it outputs position-value pairs instead of commitments to a position and to a value, which hinders its use as building block in protocols that need to protect the privacy of the prover.

Zero-Knowledge Authenticated Data Structures. Authenticated data structures (ADS) are a three-party protocol between a trusted owner, a trusted client and a server [54]. The owner uploads data to the server. The server receives queries from the client and answers them. An ADS protects data authenticity for the client when the server is adversarial.

Zero-knowledge ADS (ZKADS) provide privacy in addition to authenticity, i.e., the client does not learn anything about the data structure besides what can be inferred from the answers to the queries. The data owner is trusted. Most constructions for ZKADS do not allow the data owner to 
perform updates (see [19] and references therein). Recently, an updatable ZKADS for sets with proofs of membership [55], and an updatable ZKADS for lists, trees and partially-ordered sets of bounded dimension [20] were proposed.

Our committed database differs from ZKADS in that the prover is not split up into a trusted data owner and a server. Additionally, to our knowledge, security for ZKADS has not been defined in the UC framework. Also existing constructions are not oblivious. They reveal to clients the answers to queries in the clear, instead of revealing commitments to the answers, which hinders their use in privacy-preserving applications.

ZK proofs for large datasets. Applications of a committed database usually involve large amounts of data to be stored. Therefore, it is essential that the efficiency of protocols for reading and writing into the database be sub-linear in the database size. We recall that one of our requirements is to hide from the verifier the positions read from or written into the database. Depending on the technique used to maintain a database, the witness to compute a zero-knowledge proof for reading or writing could include all the values in the database and not just the value read or written, i.e., the size of the witness could grow with the size of the database.

Most techniques for zero-knowledge proofs are unsuitable in our case because their computation and communication cost grows linearly with the size of the witness $w$. However, some techniques exist that incur cost sub-linear in $|w|$ and that thus are appealing for computing zero-knowledge proofs about large datasets. In the following, we discuss these techniques and we show that our protocol for a committed database based on vector commitments outperforms them. We consider a prover that possesses a large dataset of size $|M|$ and that wishes to prove to a verifier that there is an element in $M$ that fulfills some statement without revealing the position of the element.

- Probabilistically Checkable Proofs (PCP) [40]. PCPs techniques produce proofs that can be verified with cost sublinear (logarithmic) in $|M|$. However, the cost for the prover is linear in $|M|$. Additionally, if the prover wishes to prove several statements about $M$, the cost is linear in $|M|$ for each proof.

- Succint Non-Interactive Arguments of Knowledge (SNARK) [41]. SNARKs produce arguments that can be verified with cost independent of $|M|$. However, the cost for the prover is still linear in $|M|$ for each statement proven about $M$. Additionally, SNARKs use non-blackbox knowledge extraction and thus cannot be used straightforwardly in the UC framework.

- Zero-Knowledge Proofs for Oblivious RAM programs [8], [9]. Zero-knowledge proofs for relations described as ORAM programs involve an initialization phase in which the prover commits to $M$. In [8], the cost for the prover is linear in $|M|$, whereas the cost for the verifier is independent of $|M|$. After the initialization phase, many proofs can be computed about $M$ whose cost is sublinear (proportional to the runtime of the ORAM program) both for the prover and for the verifier. The protocol in [8] uses a non-programmable random oracle, which is key for achieving constant cost for the verifier in the initialization phase. Any protocol in the standard model would involve communication cost linear in $M$ to allow knowledge extraction.

To compare our protocol with [8], we consider that the prover first writes $M$ into $\mathrm{Tbl}_{c d}$, and after that reads values in $M$ from $\mathrm{Tbl}_{c d}$. The cost of writing $M$ into $\mathrm{Tbl}_{c d}$ is linear in $|M|$ for both prover and verifier. However, after that the cost of each read operation is independent of $|M|$ for both prover and verifier. The reason is that zero-knowledge proofs about one vector component can be computed with cost independent of the size of the vector. There are several constructions of vector commitments, whose security is based on assumptions such as CDH, RSA or DHE. In Section VI, we show a construction based on DHE that leads to practical zero-knowledge protocols for reading from and writing into the vector commitment of cost independent of the size $|M|$ of the committed vector.

Our protocol provides thus better asymptotic amortized cost than the state of the art protocol in [8]. (In the initialization phase, the cost for the verifier is linear in $|M|$, which is unavoidable when aiming for security in the standard CRShybrid model.) We note that [8] does not provide a concrete instantiation or efficiency analysis of their protocol, so we do not compare it with the instantiation of our protocol in Section VI. 